\title{
Optimal design and analysis of clinical trials with repeated measures
}

Citation for published version (APA):

Winkens, B. (2005). Optimal design and analysis of clinical trials with repeated measures. [Doctoral Thesis, Maastricht University]. https://doi.org/10.26481/dis.20051123bw

Document status and date:

Published: 01/01/2005

DOI:

10.26481/dis.20051123bw

Document Version:

Publisher's PDF, also known as Version of record

\section{Please check the document version of this publication:}

- A submitted manuscript is the version of the article upon submission and before peer-review. There can be important differences between the submitted version and the official published version of record.

People interested in the research are advised to contact the author for the final version of the publication, or visit the DOI to the publisher's website.

- The final author version and the galley proof are versions of the publication after peer review.

- The final published version features the final layout of the paper including the volume, issue and page numbers.

Link to publication

\footnotetext{
General rights rights.

- You may freely distribute the URL identifying the publication in the public portal. please follow below link for the End User Agreement:

www.umlib.nl/taverne-license

Take down policy

If you believe that this document breaches copyright please contact us at:

repository@maastrichtuniversity.nl

providing details and we will investigate your claim.
}

Copyright and moral rights for the publications made accessible in the public portal are retained by the authors and/or other copyright owners and it is a condition of accessing publications that users recognise and abide by the legal requirements associated with these

- Users may download and print one copy of any publication from the public portal for the purpose of private study or research.

- You may not further distribute the material or use it for any profit-making activity or commercial gain

If the publication is distributed under the terms of Article $25 \mathrm{fa}$ of the Dutch Copyright Act, indicated by the "Taverne" license above, 


\section{Optimal design and analysis of \\ clinical trials with repeated measures}

by

Bjom Winkens 
Bjom Winkens, Maastricht 2005

ISBN 90-5681-232-7

The study presented in this thesis was performed at the Care and Public Health Research Institute (Caphri), which participates in the Netherlands School of Primary Care Research (accredited by the Royal Dutch Academy of Sciences (KNAW)).

Printing: Unigraphic, Maastricht University 


\title{
Optimal design and analysis of clinical trials with repeated measures
}

\author{
PROEFSCHRIFT
}

ter verkrijging van de graad van doctor aan de Universiteit Maastricht, op gezag van de Rector Magnificus, Prof.mr. G.P.M.F. Molls, volgens het besluit van het College van Decanen, in het openbaar te verdedigen op woensdag 23 november 2005 om 14:00 uur

door

Bjorn Winkens 
Promotor:

Prof.dr. M.P.F. Berger

\section{Co-promotoren:}

Dr. G.J.P. van Breukelen

Dr. HJ.A. Schouten

\section{Beoordelingscommissie:}

Prof.dr. M.H. Prins (voorzitter)

Dr. M.J.J.M. Candel

Prof dr. J.A. Knottnerus

Prof.dr. G. Molenberghs (Universiteit Hasselt)

Prof.dr. Th. Stijnen (Erasmus Universiteit Rotterdam) 


\section{Acknowledgements}

This dissertation is the result of a process of leaming and growing that would be impossible without the guidance, support, and caring of a number of persons. First of all, I would like to thank my supervisor Martijn Berger and co-supervisors Hubert Schouten and Gerard van Breukelen. Martijn's comments, advice, and especially, his encouragement to be more assertive during our meetings helped me to improve my thesis and to grow as a person. Hubert, your endless patience and well-considered, always correct comments on my work stimulated my independent thinking and research skills. I am very grateful for working with Gerard, who knows every thing about statistics a person is capable of knowing.

I thank Valeria Lima Passos, my roommate, for correcting and flourishing my English and for being a Brazilian! Valeria, I will always miss our Limburg-Brazilian conversations, lively and never-ending.

During an internship at the department of Methodology and Statistics of Maastricht University, Math Candel was my supervisor. Math, without your support and guidance I would have never become a PhD-student. Thanks to Frans Tan for the discussions about the statistical problems we encountered. It really helped me to understand the essence of these problems. I thank Marga Doyle for all her assistance, and all my colleagues for creating a stimulating environment.

I am very grateful to my parents, for everything they have done for me and to my brothers, Marco and Maurice, for their support and big brother advices. Last but not least, I would like to thank my wife, Aline, for making me the happiest man on earth by marrying me and giving birth to our beautiful baby girl, Isis. 


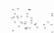

3

$\therefore$ 


\section{Contents}

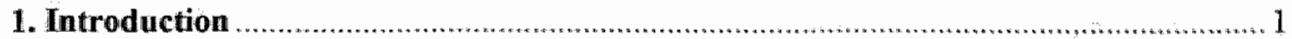

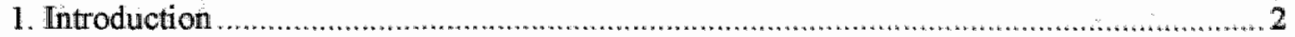

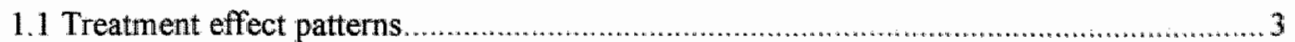

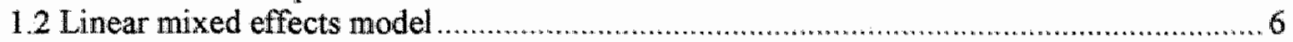

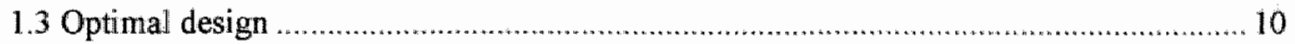

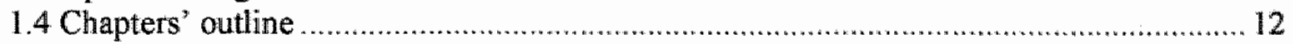

2. ANCOVA versus GLS in randomized clinical trials with a pre- and a post-treatment

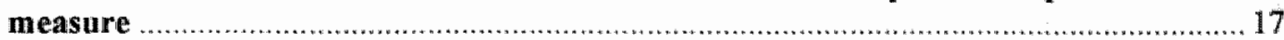

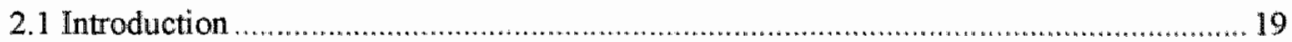

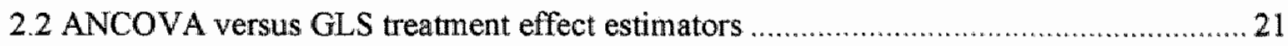

2.2.1 Situation I: homogeneous slopes and variances .............................................. 23

2.2.2 Situation $\mathrm{II}$ : heterogeneous slopes and homogeneous variances ............................ 23

2.2.3 Situation III: homogeneous slopes and heterogeneous variances ............................ 24

2.2.4 Situation IV; heterogeneous slopes and variances ........................................... 25

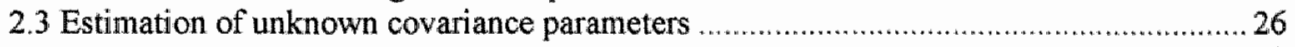

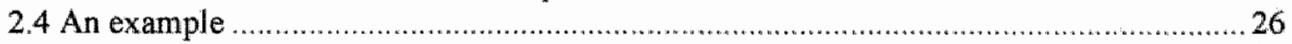

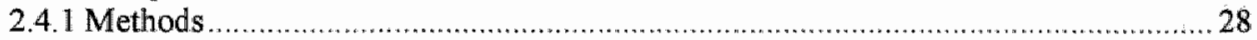

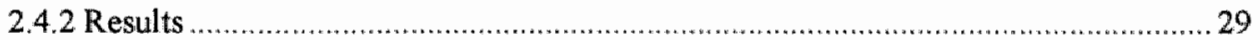

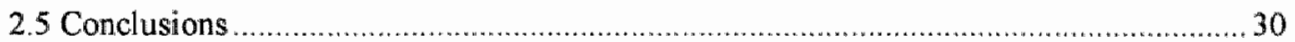

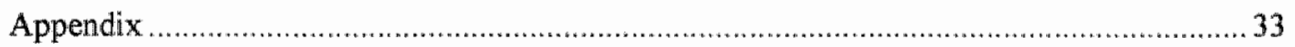

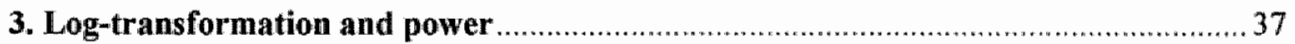

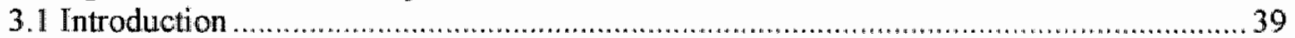

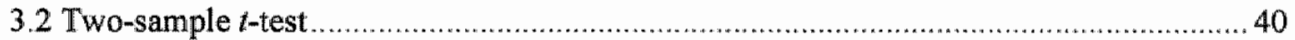

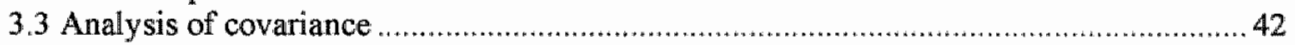

3.4 Analysis of variance for more than two groups ...................................................... 45

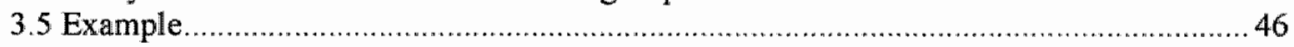

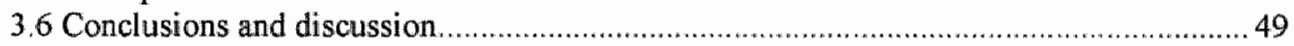

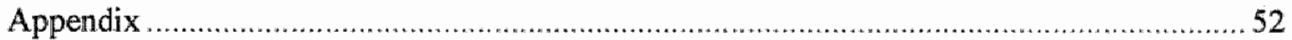

4. Optimal designs in clinical trials with linearly divergent treatment effects ............... 57

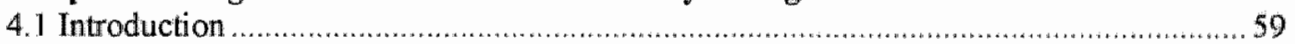

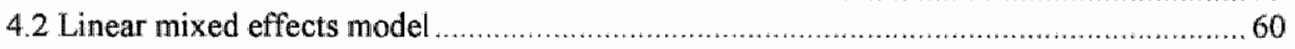

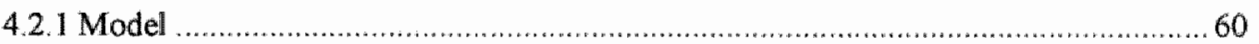

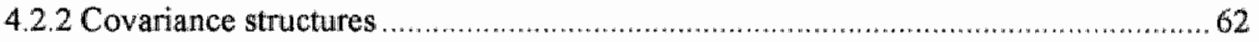

4.3 Variance of GLS treatment effect estimator .....................................................6. 65

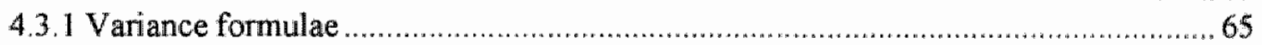

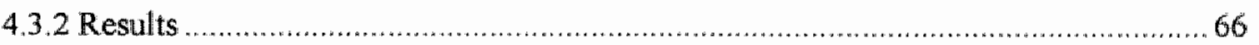

4.4 Optimal number of repeated measures and sample sizes ............................................ 68

4.4.1 Cost function ......................................................................................... 68

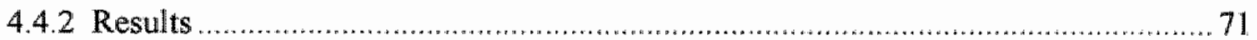

4.5 Examples: Optimal designs and misspecification of the covariance structure ...............72

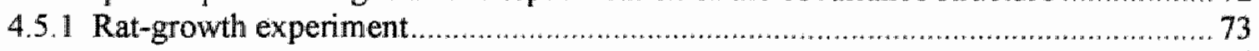

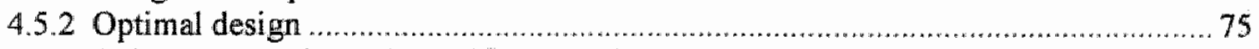

4.5.3 Robustness against misspecification of the covariance structure ........................76

4.6 Discussion and conclusions .................................................................... 76

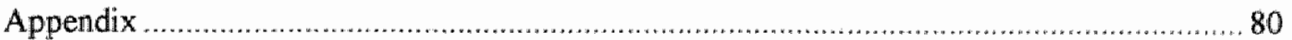


5. Optimal time-points in clinical trials with linearly divergent treatment effects

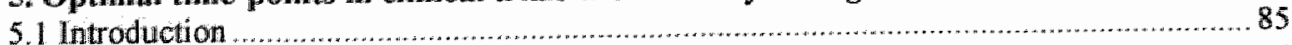

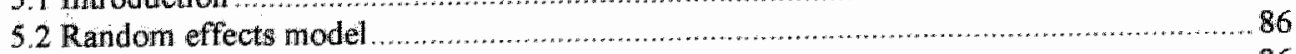

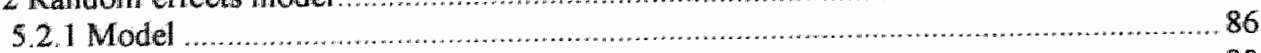

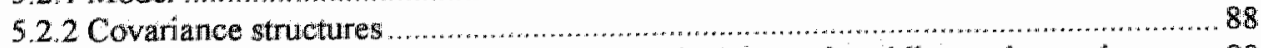

53 Optimal allocation of time-points and relative efficiency of equidistant time-points........ 89

5.3.1 Compound symmetry versus first-order auto-regression .......................................90

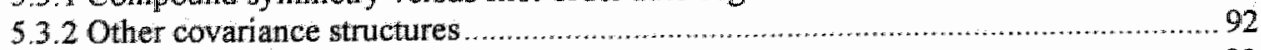

5.4 Effect of adding optimally spaced measures ........................................................... 93

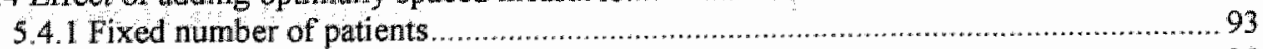

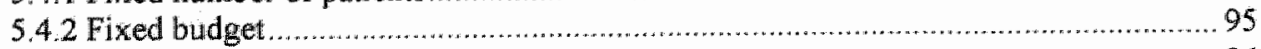

5.5 Misspecification of covariance structure............................................................. 96

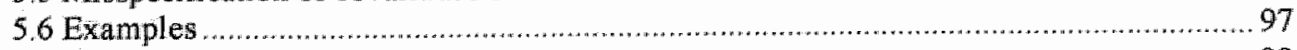

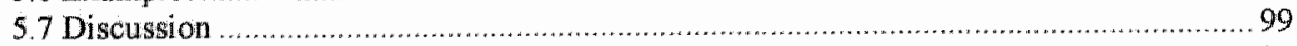

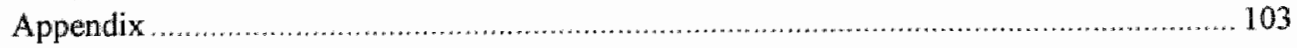

6. Optimal designs for clinical trials with second-order polymomial treatment effects 105

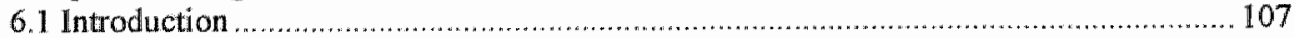

6.2 Mixed effects model and optimal design criteria ................................................... 108

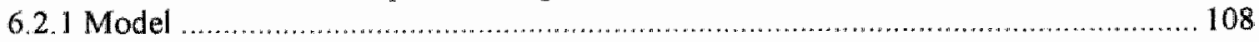

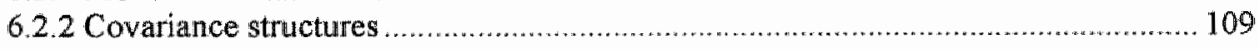

6.2 .3 Optimality criteria ............................................................................... 111

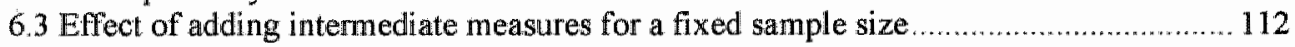

6.4 Optimal group sizes and number of measures for a fixed budget ................................ 114

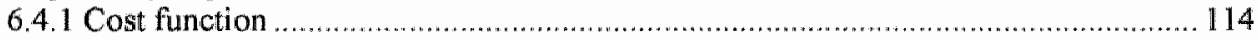

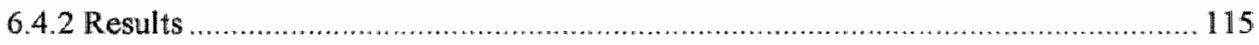

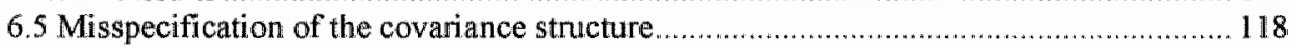

6.6 Optimal allocation of time-points for the three measures design............................... 119

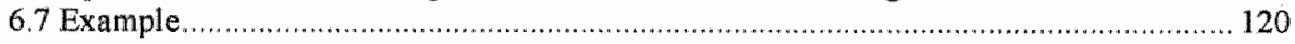

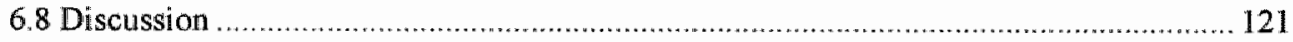

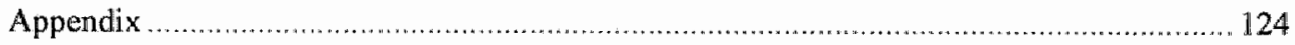

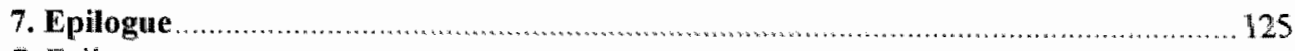

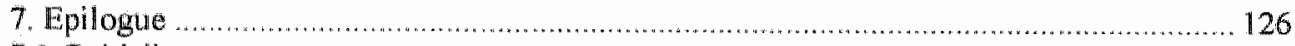

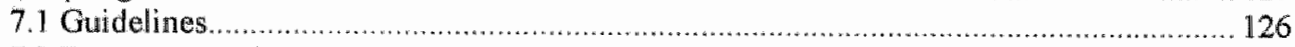

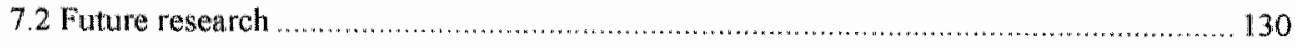

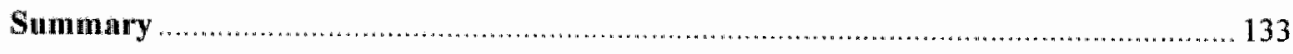

Samenvatting in het Nederlands (Dutch summary) ......................................... 137

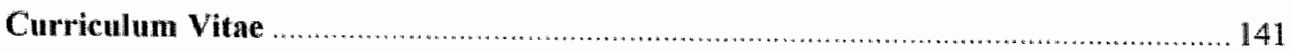


Introduction

Introduction 


\section{Chapter 1}

\section{Introduction}

This thesis deals with the optimal design and analysis of randomized clinical trials with repeated measures. Randomized clinical trials are controlled experiments designed to compare the effectiveness of different treatments over time, where subjects (patients) are randomly allocated to one of the treatment groups (control, placebo or active treatment). Subjects randomized to a control group receive a commonly used, standard treatment, whereas the subjects in an active treatment group are given a treatment that is less orthodox. If no standard treatment exists, an active treatment is often compared with a placebo. An outcome variable of interest is often measured repeatedly on the same subject to compare the different treatment effects over time.

A design for a randomized clinical trial with repeated measures may vary on the following characteristics: the number of groups, the group sizes, the number of repeated measures per subject, the allocation of time-points at which the measures are taken, among others. An important design issue is the required sample size to obtain efficient treatment effect estimators, i.e. with a small variance. If the number of repeated measures per subject increases, fewer subjects may be required to obtain this goal (e.g. Maxwell, 1998; Raudenbush \& Liu, 2001; Yi \& Panzarella, 2002). In practice, however, the number of subjects and number of repeated measures may be restricted by budget constraints and an optimal combination of the sample size and number of repeated measures per subject can be chosen to maximize the efficiency of the treatment effect estimator given a maximal budget. Usually, repeated measures are assumed to be equidistant, but this does not always yieid the most efficient treatment effect estimators (e.g. Ouwens, Tan \& Berger, 2002; Tan \& Berger, 1999). Next to the sample size and number of repeated measures, the allocation of time-points can be chosen optimally at the design stage of a clinical trial. A design with maximal efficient treatment effect estimators is called an optimal design.

Several simple and advanced analysis methods are available to compare treatment groups in repeated measures studies (see e.g. Everitt, 1995; Matthews et al., 1990; Senn, Stevens \& Chaturvedi, 2000). The selection of an efficient design depends on the analysis model, which in turn depends on the anticipated pattern of treatment effect and the covariance structure of the outcome variable (e.g. Frison \& Pocock, 1992, 1997; Overall \& Doyle, 1994; Schouten, 1999; Tan \& Berger, 1999).

Two treatment effect patterns are considered in this thesis and will be introduced in the next section. The selection of an optimal design will be discussed in this thesis for the linear 
mixed effects model, which will be introduced in Section 1.2. Subsequently, optimal design criteria are explained. Finally, in Section 1.4, the topics of the chapters will be presented and briefly motivated.

\section{1.. Treatment effect patterns}

In many repeated measures studies, the individual trend over time is assumed to be characterized by a first- or second-order polynomial function. Although higher-order polynomials are also possible, they are less prominent in the literature. Two treatment effect patterns will be considered in this thesis: 1) a linearly divergent treatment effect, and 2) a second-order polynomial treatment effect.

\section{Linearly divergent treatment effect}

A linearly divergent treatment effect is represented by a linearly increasing separation between an active treatment and a control or placebo group from start to end of the experiment, possibly after a transformation of the outcome variable. This means that the trends within the groups may still be curvilinear, but that the difference between the groups increases linearly over time. In Figure 1.1, two examples of this treatment effect pattern are presented.
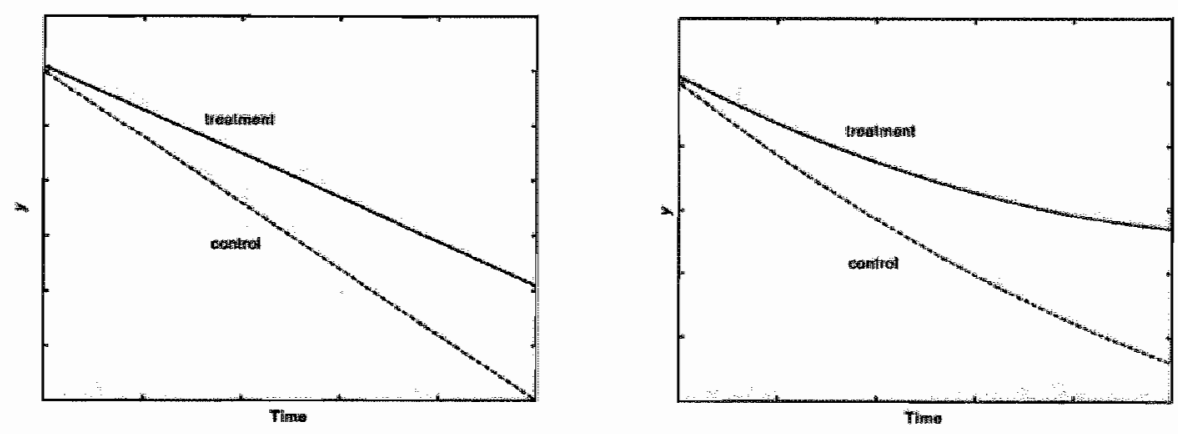

Figure 1.1 Two examples of a linearly divergent treatment effect over time.

In clinical trial research, medications are often given more than once and on a regular basis to patients during a study. In that case, a linearly divergent treatment effect may be anticipated. For example, in a 4-year randomized clinical trial (Hoffman et al., 2004), the 
effect of daily supplementation of docosahexaenoic acid (DHA) on retinal function was compared with placebo. Full-field cone electroretinograms (ERGs; the main outcome variable) were recorded at baseline and at subsequent annual visits for male patients with $\mathrm{X}$ linked retinitis pigmentosa (XLRP). Due to randomization, there was no significant group difference in $\log _{10}$ cone ERG response amplitude at baseline. Figure 1.2 shows that the group difference in $\log _{1}(E R G)$ increased linearly over time. Other examples of linearly divergent treatment effects can be found in the studies by Feighner (1980), Gruttola, Lange \& Dafni (1991) and Furberg et al (1994).

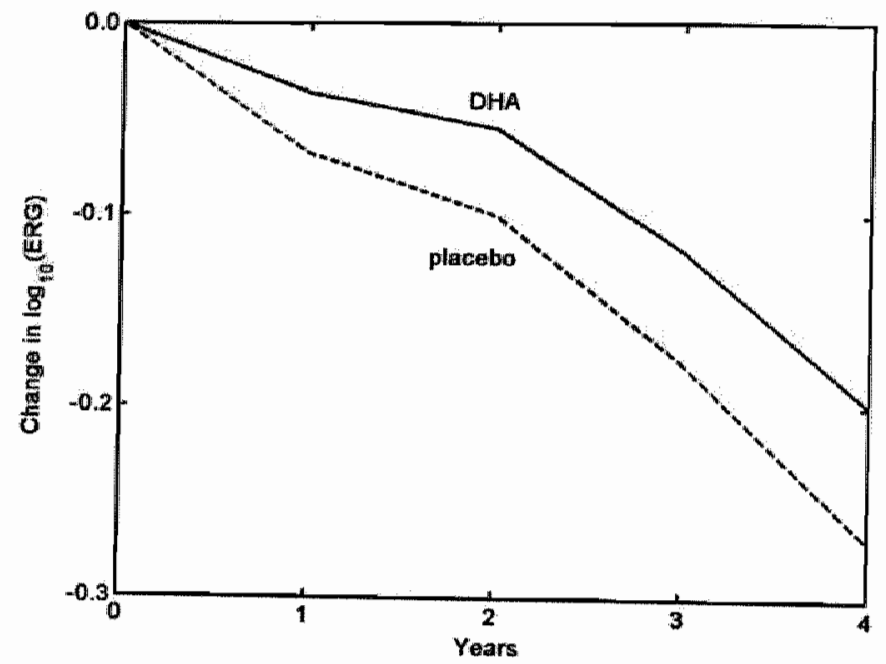

Figure 1.2 Change in $\log _{10}(E R G)$ from baseline over time (redrawn from Hoffman et al., 2004, p. 714).

\section{Second-order polynomial treatment effect}

Although a linear growth or linear change function is often seen in repeated measures studies, this pattern may not be strictly linear (see Overall \& Doyle, 1994). For example, the difference between two groups may at first increase relatively fast and then stabilize or even decrease over time when the treatment effect has reached its maximum. In Figure 1.3, such treatment effect patterns are displayed. 

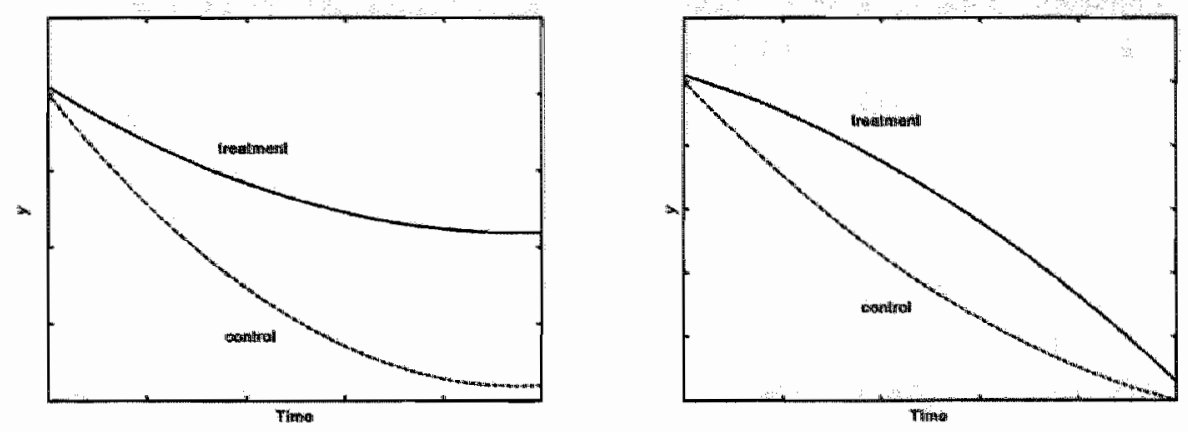

Figure 1.3 Two examples of a second-order polynomial treatment effect over time.

An example of a non-linear treatment effect is found in Agteresch et al. (2002), who reported the effect of adenosine triphosphate (ATP) on body composition, energy intake and energy expenditure in patients with advanced non-small-cell lung cancer. In the control group, a gradual decrease of mean fat mass was obtained, whereas in the ATP group, the mean fat mass first slowly decreased and then increased. Figure 1.4 displays the treatment effect, which can be represented by a second-order polynomial function. Another example of a secondorder polynomial treatment effect can be found in Omar et al. (1999, Figure 1b).

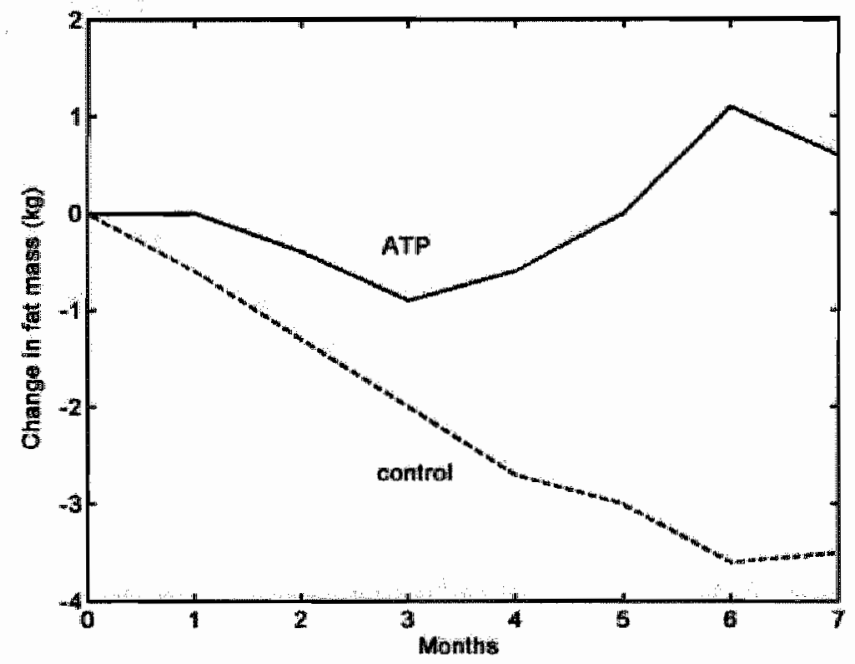

Figure 1.4 Change in fat mass from baseline over time (redrawn from Agteresch et al, 2002). 


\section{Chapter I}

The knowledge about the treatment effect pattern may be used to optimize the analysis of the data and the design of a (future) study.

\subsection{Linear mixed effects model}

Repeated measures data may require an elaborate modelling of different components of random variability, like random subject effects, serial correlation and measurement error (e.g. Diggle, Liang and Zeger, 1996, p. 79 -80). Linear mixed effects models are very flexible in modelling these various sources of variation and are therefore commonly used to analyse repeated measures data.

Let $y_{i j}$ be the jth repeated measurement for subject $i$ of a continuous outcome variable measured at time $t_{j}(i=1, \ldots, n ; j=1, \ldots, K)$. For simplicity, the time-points are assumed to be the same for each subject. Further, the first measure is taken at $t_{1}=0$, i.e. just before randomization, and the total duration of the treatment period is fixed and rescaled to unity, i.e. $t_{K}=1$. The general linear mixed effects model (e.g. Verbeke and Molenberghs, 2000, p. 28) can be written as:

$$
y_{i}=X_{i} p+Z b_{i}+\varepsilon_{i}+e_{i}
$$

where $X_{j} \beta$ models the fixed part of $y_{i}=\left(y_{i 1}, \ldots, y_{i K}\right)^{\prime}$ and $Z_{i} b_{i}+\varepsilon_{i}+e_{i}$ represents the random part of $y_{i}$. For a linearly divergent treatment effect, the outcome $y_{i j}$ has expectation $\mu_{j}$ in the control group and $\mu_{j}+\beta_{1} l_{j}$ in the treatment group, whereas the expectation of $y_{j j}$ is $\mu_{j}+\beta_{1} t_{j}+$ $\beta_{2} t_{j}^{3}$ in the treatment group for a second-order polynomial treatment effect. Due to randomization, no group effect is assumed at time $t_{1}=0$. Note that a linear mixed effects model will generally yield less efficient treatment effect estimators without this assumption (e.g. Laird and Wang, 1990). The random effects vector $b_{i}$, the component of serial correlation $\varepsilon_{i}$ and the component of measurement error $e_{i}$ are assumed to be independently, normally distributed with mean vectors 0 and covariance matrices $\boldsymbol{D}, \sigma_{\varepsilon}^{2} \boldsymbol{H}$ and $\sigma_{e}{ }^{2} \boldsymbol{I}$, respectively. Here $I$ is a $K$-dimensional identity matrix.

A graphical representation of a linear mixed effects model is given in Figure 1.5, which displays growth curves of four children in one group, who were measured yearly between 6 and 10 years. The average profile $X \beta$ over the children is presented by the bold line, child-specific protilles $\left(\boldsymbol{X}_{i} \beta+\boldsymbol{Z}_{i} \boldsymbol{b}_{i}\right)$ are presented by the thin lines and the observed lengths $y_{i j}$ of child $i$ are connected by the dotted lines. The average profile $X_{i} \beta$ is assumed to 
be linear, i.e. the fixed-effects vector $\beta=\left(\beta_{0}, \beta_{0}\right)$, where $\beta_{0}$ is the intercept (the average length of a child at age 6 ), and $\beta_{1}$ is the slope of this profile (the average linear growth over time). Further, a random intercept $b_{0 i}$ and random slope $b_{1, i}$ model was fitted to the growth curves, i.e. the random-effects vector $b_{i}=\left(b_{0,}, b_{1 i}\right)$, because the length of the children at age $6\left(\beta_{0} *\right.$ $\left.b_{0 i}\right)$ and the linear growth trend $\left(\beta_{1}+b_{1}\right) x_{j}$ may both vary between children.

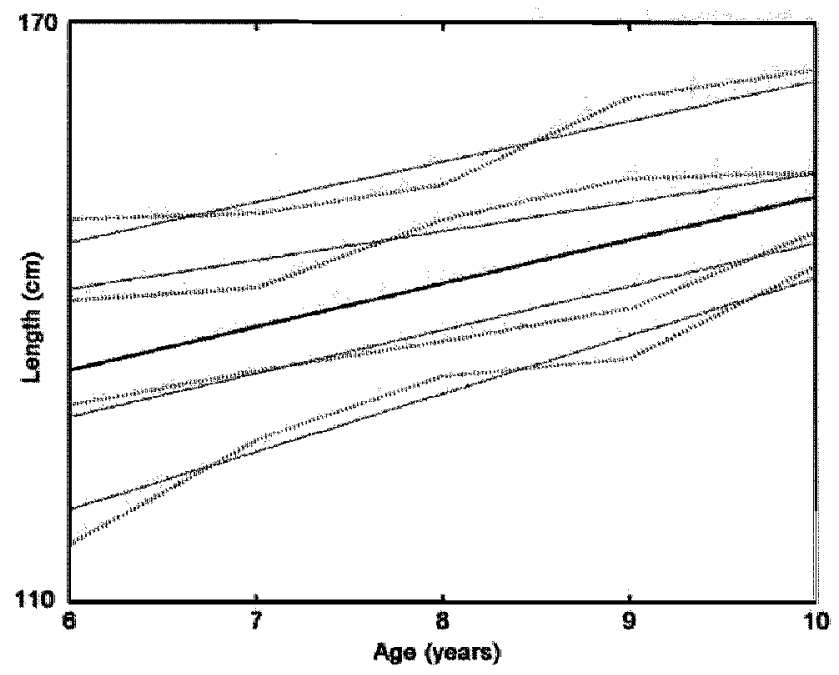

Figure 1.5 Graphical representation of a linear mixed effects model (1.1).

\section{Covariance structures}

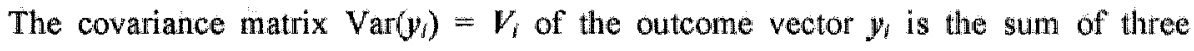
different components of variation:

1) $\operatorname{Var}\left(b_{i}\right)=Z_{i} D Z_{i}$ for random effects,

2) $\operatorname{Var}\left(\varepsilon_{i}\right)=\sigma_{s}^{2} \boldsymbol{H}$ for serial correlation,

3) $\operatorname{Var}\left(e_{i}\right)=\sigma_{e}{ }^{2} I$ for measurement error.

The between-subject variability (individual trends over time may differ from individuall to individual) is modelled by the random effects $b_{i}$ and the within-subject variability is modelled by the serial correlation component $\varepsilon_{i}$ and the measurement error component $e_{i}$ It is often suggested that at least a part $e_{i}$ of an individual's observed profile $y_{i j}(j=1, \ldots, K)$ is a response to time-varying stochastic processes operating within that individual (see e.g. Diggle, Liang and Zeger, 1996, p. 79). This type of random variation results in correlations 


\section{Chapter I}

between repeated measures, which often decrease with the time-lag $\tau=\left|t_{j}-t_{j}\right|$ between these measures. Further, the measurement process may itself add a component of variation $e_{i \text {. }}$

With these three sources of variation, quite general covariance structures may be obtained, varying from compound symmetry (CS) to first-order auto-regression (AR 1). Both structures are commonly encountered in clinical trials with repeated measures. A.CS structure is obtained by a random intercept model with measurement error. In other words, the random part of $y_{i j}$ only consists of random intercept $b_{o i}$ and measurement error $e_{i j}$, i.e. the variance $\operatorname{Var}\left(y_{i j}\right)=\operatorname{Var}\left(b_{0}\right)+\operatorname{Var}\left(e_{i j}\right)=\sigma_{0}^{2}+\sigma_{e}^{2}$ and the correlation $p_{j j^{\prime}}=\sigma_{0}{ }^{2} /\left(\sigma_{0}{ }^{2}+\sigma_{e^{2}}{ }^{2}\right)$ between $y_{i j}$ and $y_{j^{\prime}}$ are constant for all $j$ and $j^{\prime}$. An ARI structure is obtained by a fixed effects model without measurement error. In other words, the covariance matrix $V_{i}$ of $y_{i}$ is equal to $\operatorname{Var}\left(\varepsilon_{i}\right)=$ $\sigma_{\varepsilon}{ }^{2} \boldsymbol{H}$, where $\boldsymbol{H}$ has an AR 1 structure, i.e. the correlation $\rho_{\varepsilon}{ }^{\mathrm{T}}$ between $\varepsilon_{i j}$ and $\varepsilon_{l p^{p}}$ decreases with increasing time-lag $\tau=\left|t_{j}-t_{j}\right|$ and $\rho_{\varepsilon}$ is the correlation between $\varepsilon_{i 1}$ and $\varepsilon_{i K}$. Thus, both structures have a constant variance over time, but the correlation $\rho_{j j^{\prime}}$ between two repeated measures decreases with increasing time-lag $\tau$ for an ARI structure $\left(\rho_{j^{*}}=\rho_{\varepsilon}{ }^{2}\right)$ and it is constant for a CS structure $\left(\rho_{j j^{\prime}}=\sigma_{0}^{2} /\left(\sigma_{0}^{2}+\sigma_{e}^{2}\right)\right)$.

For simplicity, the covariance matrix $V_{i}$ will be assumed homogeneous across subjects and groups, i.e. $V_{i}=V$ for all subjects $i$.

\section{Estimation methods}

Several estimation methods for fixed effects have been proposed and the most commonly used is the generalized least squares (GLS) method (e.g. Diggle, Liang \& Zeger, 1996, p. 58). Generalized (or weighted) least squares estimators minimize the sum over all subjects of the weighted squared distances between the outcome variable $y_{i}$ and its expected value $X ;$, where the weights are determined by $V^{* 1}$.

For the general linear mixed effects model (1.1), the generalized least squares (GLS) estimator of the fixed effects vector $\beta$ and its variance are given by

$$
\hat{\beta}^{\text {ots }}=\left(\sum_{i=1}^{N} \boldsymbol{X}_{i}^{\prime \prime} \boldsymbol{V}^{-1} \boldsymbol{X}_{i}\right)^{-1} \sum_{i=1}^{N} \boldsymbol{X}_{i}^{\prime} \boldsymbol{V}^{-1} \boldsymbol{y}_{i}
$$

and 


$$
\operatorname{Var}\left(\hat{\boldsymbol{\beta}}^{\mathrm{cLs} s}\right)=\left(\sum_{i=1}^{N} \boldsymbol{X}_{i}^{*} \boldsymbol{V}^{-1-1} \boldsymbol{X}_{i}\right)^{-1}
$$

where $V=Z D Z+\sigma_{\varepsilon}^{2} H+\sigma_{e}^{2} I_{K}$. This GLS estimator has some nice properties. If the expectation of $y_{i}$ is correctly specified as $X i \beta$, the GLS estimator of $\beta(1.2)$ is unbiased. If the data are multivariate normally distributed, $\hat{\beta}^{\text {ats }}(1.2)$ is a maximum likelihood estimator of $\beta$ and has minimum variance (equation (1.3)) among all unbiased estimators of $\rho$ (e.g. Christensen, 1996, p. 29). In practice, however, the covariance parameters are unknown and the covariance matrix $V$ itself has to be estimated first. Two frequently used methods for estimating $V$ are maximum likelihood (ML) and restricted maximum likelihood (REML). We refer to Digglle, Liang and Zeger (1996, p. 63-68) and Verbeke and Molenberghs (2000, p. 46 49) for a comparison between ML and REML estimation.

\section{Simple analysis methods}

The linear mixed effects model is very useful for the analysis of repeated measures, but more complicated than traditional analysis methods, like analysis of covariance (ANCOVA). The difference between a linear mixed effects and an ANCOVA model can be explained as follows. Suppose each subject $i$ has been measured twice, i.e. once before treatment $(t=0)$ and once after treatment $(t=1)$. In the linear mixed effects model (1.1), the pre- and post-treatment measures are then treated as a bivariate outcome variable $\boldsymbol{y}_{i}=$ $\left(y_{i 1}, y_{i 2}\right)^{\prime}$, whereas the pre-treatment measure $y_{i}$ is used as a covariate in an ANCOVA model. An advantage of this latter is that a conditional treatment effect for a given pre-treatment value can be estimated from an ANCOVA model. Advantages of the mixed effects approach are: 1) it can easily be extended to more than two repeated measures and 2) several methods to deal with missing datta are available (e.g. Verbeke \& Molenberghs, 2000, p. 201-390). In Chapter 2, the ANCOVA and linear mixed effects model will be compared with respect to the estimation of an overall treatment effect, i.e. difference in post-treatment expectation between the groups.

The linear mixed effects and ANCOVA models assume that the data are normally distributed. If this assumption is violated, however, the actual type I error risk may be larger than a pre-specified value $\alpha$ and the power of these methods may be low. A data transformation may then be used to normalize the dlata. Positively skewed data are often encountered in practice. For example, costs of medical care or response times can only take 


\section{Chapter I}

on positive values and are often affected by positive outliers (high costs or slow response, respectively): The data may then be normalized by a log-transformation (e.g. Keene, 1995). The benefit of log-transforming positively skewed data before analyzing the data will be discussed in Chapter 3.

\subsection{Optimal design}

A randomized clinical trial with repeated measures should be designed optimally, because it generally demands a high amount of time and money. Different optimality criteria may be used and may lead to different optimal designs (e.g. Atkinson \& Donev, 1996, p. 106115). Therefore, an optimality criterion has to be chosen carefully.

Since the GLS estimator $\hat{\beta}^{\text {CLS }}$ in (1.2) is unbiased, provided the expectation of $y_{i}$ is correctly specified as $X_{i}$, an optimality criterion is only a function of its covariance matrix (1.3), which depends on the characteristics of a design, i.e. the sample size $N$, the number of repeated measures per subject $K$ and the allocation of time-points $t_{j}$ at which the measures are taken. The selection of an optimal design, i.e. choosing these characteristics optimally in terms of a specified optimality criterion, depends on the covariance structure and on the hypothesized treatment effect pattern, e.g. whether it is linearly divergent or second-order polynomial.

\section{Linearly divergen treatmem effect}

If the treatment effect pattern is assumed to be linearly divergent, the fixed effects vector is $\beta=\left(\mu_{1}, \ldots, \mu_{k}, \beta_{1}\right)^{\prime}$, where $\left(\mu_{1}, \ldots, \mu_{K}\right)$ describes the mean profile of $y_{i j}$ in the control group and $\beta_{1}$ is the treatment effect parameter, In the ERG study (Hoffman et al., 2004), for example, that was introduced in Section 1.1, the mean $\log _{10}\left(\right.$ ERG) at time $t_{j}=(j-1)$ years is $\mu_{j}$ in the placebo group and $\mu_{j}+\beta_{1} t_{j}$ in the DHA group. In this case, we are only interested in the treatment effect parameter $\beta_{1}$ and a so-called $c$-optimality criterion, i.e. $\operatorname{Var}\left(c^{\prime} \hat{\beta}^{\text {at.s }}\right)$, where $c^{\prime}=(0, \ldots, 0,1)$, can be used.

\section{Second-order polynomial treatment effect}

For second-order polynomial treatment effects, i.e. $\beta=\left(\mu_{1}, \ldots, \mu_{K}, \beta_{1}, \beta_{2}\right)^{\prime}$, we are interested in both linear and quadratic treatment effect parameters $\left(\beta_{1}\right.$ and $\left.\beta_{2}\right)$. In this thesis, two different optimality criteria will be used: 


\section{Introdiction}

1) a $D_{y}$-optimality criterion, i.e. the determinant of the covariance matrix of $\hat{\beta}_{1}^{\mathrm{GLS}}$ and $\hat{\beta}_{2}^{\mathrm{GLS}}$,

2) a $c$-optimality criterion, i.e. $\operatorname{Var}\left(c^{\prime} \hat{\beta}^{\text {oLs }}\right)$, where $c^{\prime}=(0, \ldots, 0,1 / 2,1 / 3)$.

It can be shown that if the curves of the treatment and control groups do not intersect for $0<t$ $<1$, the latter criterion corresponds to the difference between the areas under these curves $(A \cup C)$, which is often used in practice (e.g. Matthews et al., 1990; Schiff, 2003). As for the first criterion, the $D$-optimality criterion, i.e. $\operatorname{Det}\left[\operatorname{Var}\left(\hat{\beta}^{\text {ous }}\right)\right]$, is also commonly used in practice, because of its nice properties (e.g. Atkinson \& Donev, 1996, p. 106-107). For example, a $D$-optimall design is independent of the scale of the variables in the design matrices $X_{i}$ and it minimizes the area of the simultaneous confidence region of the fixed parameters $\beta$. Since we are only interested in a subset of $s=2$ parameters $\left(\beta_{1}\right.$ and $\left.\beta_{2}\right)$, however, the determinant of the covariance matrix of only their GLS estimators is minimized $\left(D_{s}\right.$-optimality criterion).

The $D_{\mathrm{s}}$ - and c-optimal designs are graphically represented in Figure 1.6, in which the simultaneous confidence regions (ellipses) of the treatment effect parameters $\beta_{1}$ and $\beta_{2}$ are plotted. The ellipses are given for a set of regression vectors $\beta_{12}=\left(\beta_{1}, \beta_{2}\right)^{\prime}$, where $\left(\boldsymbol{\beta}_{12}-\hat{\boldsymbol{\beta}}_{12}^{\text {GLS }}\right)^{\prime}\left[\operatorname{Var}\left(\hat{\boldsymbol{\beta}}_{12}^{\mathrm{OLS}}\right)\right]^{-1}\left(\boldsymbol{\beta}_{12}-\hat{\boldsymbol{\beta}}_{12}^{\mathrm{OLS}}\right) \leq \gamma$ for a specific $\hat{\boldsymbol{\beta}}_{12}^{\mathrm{GLS}}, \operatorname{Var}\left(\hat{\boldsymbol{\beta}}_{12}^{\mathrm{aLS}}\right)$ and $\gamma$, which depends on the confidence level of the region. The lengths of the longest $l_{1}$ and smallest axis $l_{2}$ of the ellipse are related to the covariance matrix $\operatorname{Var}\left(\hat{\boldsymbol{\beta}}_{12}^{\mathrm{GLS}}\right)$, which is larger in the direction of $l_{1}$ than in the direction of $l_{2}$. The intersection of the axes $l_{1}$ and $l_{2}$ is equal to the GLS estimate $\hat{\beta}_{12}^{\text {GLS }}=\left(\hat{\beta}_{1}^{\text {GLs }}, \hat{\beta}_{2}^{\text {ots }}\right)$. The covariance between the GLS estimators $\hat{\beta}_{1}^{\text {oLs }}$ and $\hat{\beta}_{2}^{\text {ots }}$ determines the directions of these axes. If these estimators are uncorrelated, the axes $t_{1}$ and $t_{2}$ are parallel to the co-ordinate axes (see Figures 1.6a and 1.6b). In this case, the length of the axes $l_{1}$ and $l_{2}$ are equal to $2 \sqrt{\gamma \times \operatorname{Var}\left(\hat{\beta}_{1}^{\text {oL.s }}\right)}$ and $2 \sqrt{\gamma \times \operatorname{Var}\left(\hat{\beta}_{2}^{\text {GL.s. }}\right)}$, respectively. In Figures $1.6 \mathrm{c}$ and $1.6 \mathrm{~d}$, the confidence ellipse is displayed where the covariance $\operatorname{Cov}\left(\hat{\beta}_{1}^{\mathrm{aLs}}{ }^{\mathrm{a}} \hat{\beta}_{2}^{\mathrm{GLS}}\right) \neq 0$

The $D_{5}$-optimal design minimizes the product of the squares of the lengths of the axes of the ellipse, which is proportional to (the square of) the area of the ellipse. Further, three projections of the ellipse are shown (dotted lines), which correspond to c-optimality criteria $\operatorname{Var}\left(c^{\prime} \hat{\beta}^{\text {ror.s }}\right)$. In Figures $1.6 \mathrm{a}$ and $1.6 \mathrm{c}$, the ellipse is projected on the $\beta_{1}$ - and $\beta_{2}$ axes. The corresponding $c$-optimality criteria are then $c_{1}{ }^{\prime \prime}=(0, \ldots, 0,1,0)$ and $c_{2}{ }^{\prime}=(0, \ldots, 0,0,1)$, respectively. Figures $\mathbb{1} .6 \mathrm{~b}$ and $1.6 \mathrm{~d}$ show the projection of the ellipse on the vector $(1 / 2,1 / 3)^{\prime}$ * 


\section{Chapter I}

which relates to the $c$-optimality criterion with $c^{n}=(0, \ldots, 0,1 / 2,1 / 3)$, i.e. the AUC-optimality criterion.

(a)

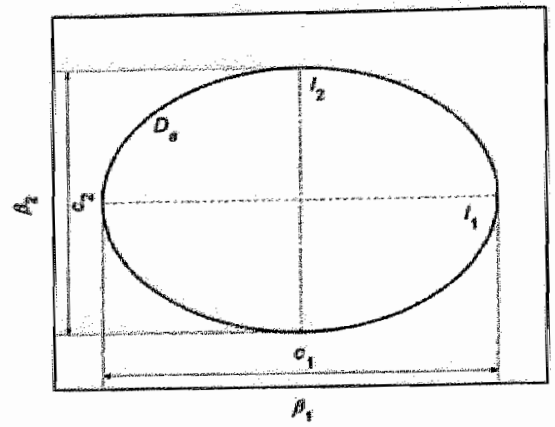

(c)

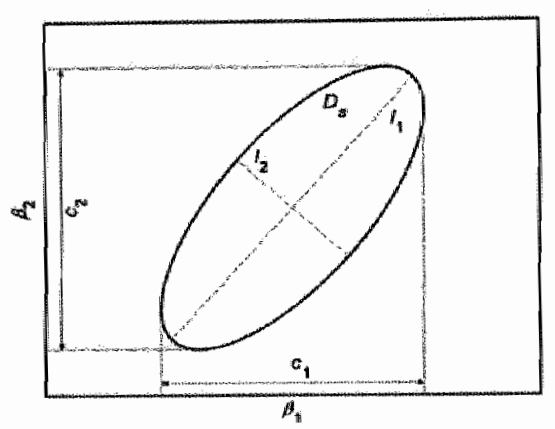

(b)

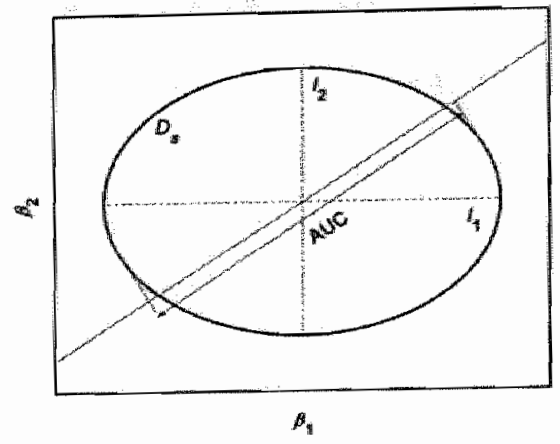

(d)

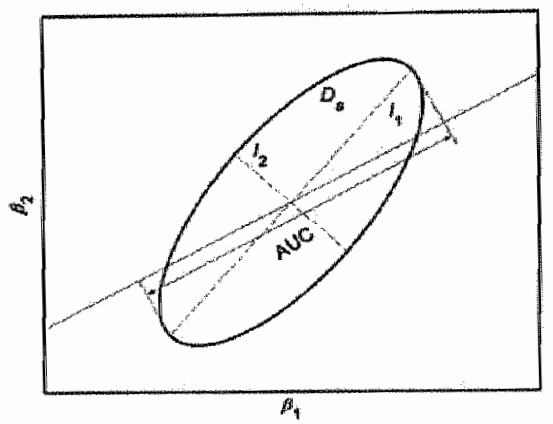

Figure 1.6 Simultaneous confidence regions (ellipses) of the treatment effect parameters $\beta_{1}$ and $\beta_{2,}$ and three projections of these ellipses, corresponding to $c$-optimality criteria: $c_{1}^{\prime}=$ $(0, \ldots, 0,1,0)_{2} c_{2}{ }^{\prime}=(0, \ldots, 0,0,1)$ and $c^{\prime}=(0, \ldots, 0,1 / 2,1 / 3)$, i.e. $A U C$-optimality criterion.

\subsection{Chapters' outline}

The chapters in this thesis may be read as self-contained papers. The notation may slightly deviate in some chapters from other chapters to increase their readability. The objectives and motivation of the chapters are as follows:

Chapter 2. The linear mixed effects approach is very useful to model repeated measures data, but this approach is more complicated than traditional analysis methods, like 


\section{Introduction}

analysis of covariance (ANCOVA). In this chapter, randomized clintcal trials are considered in which each subject is measured twice, i.e once before and once after treatment. In the linear mixed effects approach, the pre- and post-treatment measures are then considered to be a bivariate outcome variable, while the pre-treatment measure is treated as a covariate in ANCOVA. Although a conditional treatment effect for a given pre-treatment value can only be estimated with ANCOVA, both methods may estimate an overall treatment effect, ie. difference in post-treatment expectation between the groups. These two methods are then compared with respect to the estimation of an overall treatment effect for four different situations, differing in whether the regression slopes and/or residual post-treatment variances are homogeneous across groups.

Chapter 3. Many statistical analysis methods assume that the data are normally distributed. In practice, however, positively skewed data are frequently encountered due to the type of data or outliers. Costs of medical care, concentration of ozone in the air, response times and antibody concentrations after vaccination are examples of variables that can only take on positive values and are commonly affected by outliers. A log-transformation may then be used to normalize the data. In this chapter, the effect of log-transforming positively skewed data before using a simple analysis method, like two-sample t-test, analysis of variance (ANOVA) or analysis of covariance (ANCOVA), is considered. Formulae are derived for the asymptotic relative efficiencies (ARE) of these methods based on untransformed versus $\log$ transformed data:

Chapter 4. In repeated measures experiments, the required sample size to obtain efficient treatment effect estimators may be decreased by increasing the number of repeated measures per subject. In this chapter, the effect of adding intermediate measures is considered for six different covariance structures, assuming a linearly divergent treatment effect, equidistant time-points and either a fixed sample size or a fixed study budget. Formulae for the variance of the generalized least squares (GLS) treatment effect estimator are derived for different covariance structures. For a fixed study budget, the optimal combination of sample size and number of repeated measures per subject is computed by minimizing this variance subject to a linear cost function. Since the results depend on the assumed covariance structure, which is generally unknown at the design stage of a study, misspecification of the cowariance structure is also addressed. The numerical results are illustrated by three examples.

Chapter 5. This chapter deals with an extension to the work in the previous chapter. Next to sample size and number of repeated measures per subject, the allocation of timepoints can be chosen optimally to minimize the costs of a study. The optimal allocation of 


\section{Chapter 1}

time-ponts is obtained numerically for randomized clinical trials with a small number of repeated measures and linearly divergent treatment effects. The relative efficiency of equidistant versus optimally allocated time-points, defined as the inverse ratio of the variances of the GLS treatment effect estimators, is then calculated for different covariance structures: Also, the effect of adding intermediate, optimally spaced measures on the variance of the GLS treatment effect estimator is computed, assuming either a fixed number of subjects or a fixed study budget. The results strongly depend on the assumed covariance structure. Therefore, misspecification of the covariance structure is also addressed. For two examples, the optimal designs are calculated and compared with the used designs, assuming a fixed. budget and a linetur cost function.

Chapter 6. Chapters 4 and 5 deal with optimal design issues for randomized clinical trials in which the treatment effect is linearly divergent. However, the selection of an optimal design depends on the pattern of treatment effect and this pattern may not be linear, even after a data transformation. Therefore, in this chapter, optimal designs are computed for a secondorder polynomial instead of a linearly divergent treatment effect. The efficiency of treatment effect estimation is discussed for equidistant time-points, different covariance structures and two optimality criteria $\left(D_{s}\right.$ and $\left.A U C\right)$, assuming either a fixed sample size or a fixed study budget. Misspecification of the covariance structure is also addressed. For a design with three repeated measures, the optimal allocation of time-points is calculated and compared with equidistant time-points with respect to the efficiency of treatment effect estimation.

Chapter 7. In this chapter (epilogue), the results from the previous chapters are discussed and guidelines regarding the design and analysis method are offered to practitioners. 


\section{Introduction}

\section{References}

Agteresch, H. J., Rietveld, T., Kerkhofs, L. G. M. Van den Berg, J. W. O., Wilson, J. H. P., \& Dagnelie, P. C. (2002). Beneficial effects of adenosine triphosphate on nutritional. status in advanced lung cancer patients: A randomized clinical trial, Jownal of Clinical Oncology, 20, 371-378.

Atkinson, A. C., \& Donev, A. N. (1996). Optimum Experimental Designs. Oxford: Clanendon Press.

Christensen, R. (1996). Plane Answers to Complex Questions: The Theory of Limear Models. New York: Springer-Verlag.

Diggle, P. J., Liang, K-Y, \& Zeger, S. L. (1996). Analysis of Longitudinal Data. Oxford: Clarendon Press.

Everitt, B. S. (1995). The analysis of repeated measures: a practical review with examples, The Statistician, 44, 113-135.

Feighner, J. P. (1980). Trazodone, a triazolopyridine derivative, in primary depressive disorder, Journal of Clinical Psychiatry, 41, 250-255

Frison, L., \& Pocock, S. J. (1992). Repeated measures in clinical trials: analysis using mean summary statistics and its implications for design, Siatistics in Medicine, 11, 16851704.

Frison, L. \& Pocock, S. J. (1997) Linearly divergent treatment effects in clinical trials with repeated measures: efficient analysis using summary statistics, Statistics in Medicine, 16, $2855-2872$.

Furberg, C. D., Adams, H. P., Applegate, W. B., et al (1994). Corony heart disease /myocardial infarction: Effect of Lovastatin on early carotid atherosclerosis and cardiovascular events, Circulation, 90, 1679-1687.

Gruttola, V., Lange, N., \& Dafni, U. (1991). Modeling the progression of HIV infection, Journal of the American Statistical Association, 86, 569-577.

Hoffiman, D. R., Locke, K. G., Wheaton, D. H., Fish, G. E., Spencer, R., Birch, D. G. (2004). A randomized, placebo-controlled clinical trial of docosahexaenoic acid supplementation for $\mathrm{X}$-linked retinitis pigmentosa, American Journal of Ophthalmology, 137, 704-718.

Keene, O. N. (1995). The log transformation is special, Staristics in Medicine, 14, 811-819. Laird, N. M., \& Wang, F. (1990). Estimating rates of change in randomized clinical trials, Controlled Clinical Trials, 11,405-419. 


\section{Chapter 1}

Matthews, I. N. S., Altman, D. G, Campbell, M. J ; \& Royston, P. (1990). Analysis of serial measurements in medical research, British Medical Journal, 300, 230-235.

Maxwell, S. E. (1998). Longitudinal designs in randomized group comparisons: when will intermediate observations increase statistical power?, Psychological Methods, 3, 275290.

Omar, R Z, Wright, E. M., Tumer, R. M., \& Thompson, S. G. (1999). Analysing repeated measurements data: a practical comparison of methods, Statistics in Medicine, 18, 1587-1603.

Ouwens, M. J. N. M. Tan, F. E. S. \& Berger, M. P. F. (2002). Maximin D-optimal designs for longitudinal mixed effects models, Biometrics, 58, 735-741.

Overall, J. E., \& Doyle, S. R. (1994). Estimating sample sizes for repeated measurement

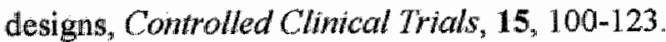

Raudenbush, S. W., \& Liu, X-F. (2001). Effects of study duration, frequency of observation, and sample size on power in studies of group differences in polynomial change, Psychological Methods, 6, 387-401.

Schiff, M. (2003). A rationale for the use of summary measurements for the assessment of the effects of rheumatoid arthritis therapies, Clinical Therapewtics, 25, 993-1001.

Schouten, H. J A. (1999). Planning group sizes in clinical trials with a continuous outcome and repeated measures, Statistics in Medicine, 18, 255-264.

Senn, S., Stevens, L, \& Chaturvedi, N. (2000). Repeated measures in clinical trials: simple strategies for analysis using summary measures, Statistics in Medicine, 19, 861-877.

Tan, F. E. S. \& Berger, M. P. F. (1999). Optimal allocation of time points for the random effects model, Communications in Statistics, Simulations and Computations, 28, 517 540.

Verbeke, $\mathrm{G}$. \& Molenberghs, G. (2000). Linear Mixed ModeLs for Longitudinat Data. New York: Springer-Verlag.

Yi, Q, \& Panzarella, T. (2002). Estimating sample size for tests on trends across repeated measurements with missing data based on the interaction term in a mixed model, Controlled Clinical Trials, 23, $481-496$. 
2 ANCOVA versus GLS in randomized clinical trials 


\section{Chapler 2}

\section{Abstract}

In a randomized clinical trial, analysis of covariance (ANCOVA) may be used to calculate a conditional treatment effect, i.e. difference in post-treatment means for a given pre-treatment value. The alternative method, generalized least squared (GLS), is to treat the pre-and post-treatment measures as a bivariate response variable, where the treatment only affects the post-treatment measure. This method estimates an overall treatment effect, i.e. difference in post-treatment expectation between two groups. In this paper, ANCOVA is compared with GLS with respect to the overall treatment effect estimators and their variances. We conclude that ANCOVA is preferred to GLS when regression slopes are homogeneous, because of unbiased variance estimators, irtespective whether residual variances are homogeneous or heterogeneous. In case of heterogeneous slopes and homogeneous residual variances, GLS could not be applied with the present software and ANCOVA must be used. In case the slopes and residual variances are both heterogeneous, GLS with Kenward and Roger's adjusted variance estimator is preferred to estimate an overall treatment effect, because it takes into account the variability of the pre-treatment mean estimator. In case of heterogeneous slopes, however, a conditional treatment effect may be more informative than the overall treatment effect, which again requires ANCOVA. The results of this comparison are illustrated with an example.

Keywords: Analysis of Covariance (ANCOVA); Generalized Least Squares (GLS); Kenward and Roger's Adjustment; Randomized Clinical Trials; Repeated Measures. 


\subsection{Introduction}

Analysis of covariance (ANCOVA) is often applied in randomized clinical trials to estimate a conditional treatment effect, i.e. the difference in two post-treatment means for a given pre-treatment value. Two main purposes of ANCOVA are reduction of error variance in randomized clinical trials (e.g. Senn, 1997) and adjustment for initial differences in outcome between treatment groups in non-randomized clinical trials; see Maris (1998) for great problems with the latter purpose. The all ternative method is to treat the repeated measures as a bivariate response variable, where the treatment only affects the post-treatment measures. This alternative method fits in a generalized least squares (GLS) framework, which is used in generalized estimating equations (GEE) (e.g. Liang \& Zeger, 1986) and mixed models (e.g. Verbeke \& Molenberghs, 2000), and estimates an overall treatment effect, i.e. difference in post-treatment expectation between two groups. In case of heterogeneous regression slopes, however, the treatment effect depends on the pre-treatment value. Therefore, condilional treatment effects are more informative than an overall treatment effect and ANCOVA should be used (e.g. Fleiss, 1986, p. 204). The overall treatment effect can still be useful for patients with unknown pre-treatment value or for a general conclusion about a treatment. In case the regression slopes are homogeneous, the treatment effect does not depend on the pre-treatment value,

Although the overall treatment effect estimators and their variances are known for ANCOVA in most situations, they will be compared in the present paper with the GLS ones in four different situations, differing in whether the regression slopes and/or residual posttreatment variances are homogeneous across groups.

Consider a randomized study of $G$ treatments and a quantitative outcome variable $y$. In group $g$, subject $\hat{x}$ has a pre-treatment measurement $y_{g n}$ at time $t=0$ and a post-treatment measurement $y_{g i z}$ at time $t=1, g=1,2, \ldots, G$ and $i=1,2, \ldots, N_{g}$. Because of the randomization all groups are assumed to have the same pre-treatment expectation $\mu_{1}$ and variance $\sigma_{1}{ }^{2}$. The within-group post-treatment expectation $\mu_{g 2}$ and variance $\sigma_{g ; 2^{2}}{ }^{2}$ as well as the within-group covariance between pre- $(t=0)$ and post-treatment $(t=1)$ measures $\sigma_{g ;}$ may be influenced by the treatments, indicated by subscript $g$. A bivariate normal distribution is assumed with expectation $\mu_{g}=\left(\mu_{1}, \mu_{g 2}\right)^{\prime}$ and 2 by 2 covariance matrix $V_{g}$, i.e. 


$$
\left(\begin{array}{l}
y_{g / 1} \\
y_{g ; 2}
\end{array}\right)-N\left(\mu_{g}, \nu_{g}\right)=N\left(\left(\begin{array}{c}
\mu_{1} \\
\mu_{g^{\prime} 2}
\end{array}\right),\left(\begin{array}{cc}
\sigma_{1}^{2} & \sigma_{g ; 2} \\
\sigma_{g: 12} & \sigma_{g ; 2}^{2}
\end{array}\right)\right)
$$

The (overall) treatment effect between groups $g$ and $g$ is defined as the difference in posttreatment expectations, i.e. $\tau_{g_{g}}=\mu_{g_{2}}-\mu_{g 2}$.

The non-parallel lines (heterogeneous regression slopes) ANCOVA model can be derived from equation (2.1) by writing it as a conditional model,

$$
y_{g 22}=\mu_{g 2}+\gamma_{g}\left(y_{g i 1}-\mu_{1}\right)+e_{g i 2}
$$

where $\gamma_{g}$ is the group-specific slope of the regression line of group $g$. Furthermore, the errors $e_{g / 2}$ are normally and independently distributed with mean zero and variance $\sigma_{\theta_{g}}^{2}=\left(1-\rho_{g: 12}^{2}\right) \sigma_{g: 2}^{2}$, where $\rho_{g: 12}$ is the within-group correlation between pre- and posttreatment measures of group $\mathrm{g}$. Since the pre-treatment measurement in model (2.2) is written as a deviation from the population mean, $\left(y_{g i 1}-\mu_{1}\right), \mu_{g 2}$ is the conditional expectation for a subject with an average pre-treatment value, but it is also the unconditional expectation of $y_{g i 2}$. Furthermore, the within-group regression slope $\gamma_{g}=\sigma_{g ; 12} / \sigma_{1}{ }^{2}$. The parallel lines ANCOVA model assumes, additional to homogeneous residual variances, that the slopes of all groups are equal, i.e. $\gamma_{1}=\ldots=\gamma_{G}=\gamma$. In this case, the mean $\mu_{1}$, which is generally unknown, can be omitted from the model without affecting the treatment effect estimators.

Equation (2.1) can also be written as a repeated measures model,

$$
y_{g i j}=\mu_{1}\left(1-t_{j}\right)+\mu_{g 2} t_{i}+\varepsilon_{g i t}^{*}
$$

where $\left.t_{j}=0,10=1,2\right)$. The errors of subject $j$ in group $g, \varepsilon_{g i n}$ and $\varepsilon_{g i 2}$, are assumed to be bivariate normally distributed with mean vector $\theta$ and covariance matrix $V_{z}$. Additionally, the errors of different subjects are assumed to be statistically independent.

In Section 2.2, ANCOVA treatment effect estimators and variances are discussed for four different situations, differing in whether the regression slopes and/or residual posttreatment variances are homogeneous across groups. Equivalent assumptions within the GLS framework will also be discussed. The GLS treatment effect estimators are compared to those of ANCOVA in case of known and unknown covariance parameters. In Section 2.3, 
estimation methods for unknown covariance parameters are discussed. The results of the comparison of ANCOVA and GLS are illustrated with a real-life example in Section 2.4. Finally, the results are summarized and discussed in Section 2.5 .

\subsection{ANCOVA versus GLS treatment effect estimators}

In case of known $\sigma_{1}{ }^{2}, \sigma_{g, 12}, \sigma_{g:{ }^{2}}$ and unknown $\mu_{1}$, ANCOVA and GLS lead to the same treatment effect estimators (Laird \& Wang, 1990), i.e.

$$
\hat{\tau}_{g, g^{\prime}}=\left\{\begin{array}{cc}
\left(\bar{y}_{g, 2}-\bar{y}_{g^{\prime}, 2}\right)-\gamma\left(\bar{y}_{g \cdot 1}-\bar{y}_{g^{\prime}, 1}\right) & \text { if homogeneous slopes, } \\
\left.\left(\bar{y}_{g, 2}-\bar{y}_{g^{\prime}, 2}\right)-\gamma_{g}\left(\bar{y}_{g, 1}-\bar{y}_{.1}\right)-\gamma_{g^{\prime}}\left(\bar{y}_{g^{\prime}, 1}-\bar{y}_{.1}\right)\right\} & \text { if heterogeneous slopes, }
\end{array}\right.
$$

where $\bar{y}_{g, 1}$ and $\bar{y}_{g .2}$ are the within-group pre- and post-treatment means of group $g$, respectively. Further, $\bar{y}_{.1}=\left(\Sigma_{y} \Sigma_{i} y_{g i l}\right) / N$ is the overall pre-treatment mean, where $N$ is the total number of subjects in the experiment. The ANCOVA and GLS variance estimators of the overall treatment effects (see Table 2.1) are, however, different when the regression slopes $\gamma_{g}$ are assumed to be heterogeneous (situations II and IV). The difference between the GLS and ANCOVA variance estimators, i.e. $\left(\sigma_{g: 12}-\sigma_{g^{\prime}: 12}\right)^{2} /\left(N \sigma_{1}^{2}\right)$, is a correction factor for unknown $\mu_{1}$ and is equal to 0 , when the regression slopes $\gamma_{\mathrm{g}}$ are homogeneous. The ANCOVA variance estimator does not contain a correction factor, because in an ANCOVA model $y_{g i a}$ is modelled conditional on $y_{g_{\text {gt }}}$. Therefore, assuming known $\sigma_{1}{ }^{2}, \sigma_{g_{1}}{ }_{2}, \sigma_{g_{1}}{ }^{2}$, unknown $\mu_{1}$ and random $y_{g 1,}$, ANCOVA variance estimators are generally biased downwards in case of heterogeneous regression slopes. 
Table 2.1 Estimated variances of ANCOVA and GLS treatment effect estimators for known or unknown covariance parameters, but unknown $\mu_{0}$. Regression slopes and residual variances are: I - both homogeneous; II - heterogeneous and homogeneous, respectively; III homogeneous and heterogeneous, respectively; IV - both heterogeneous.

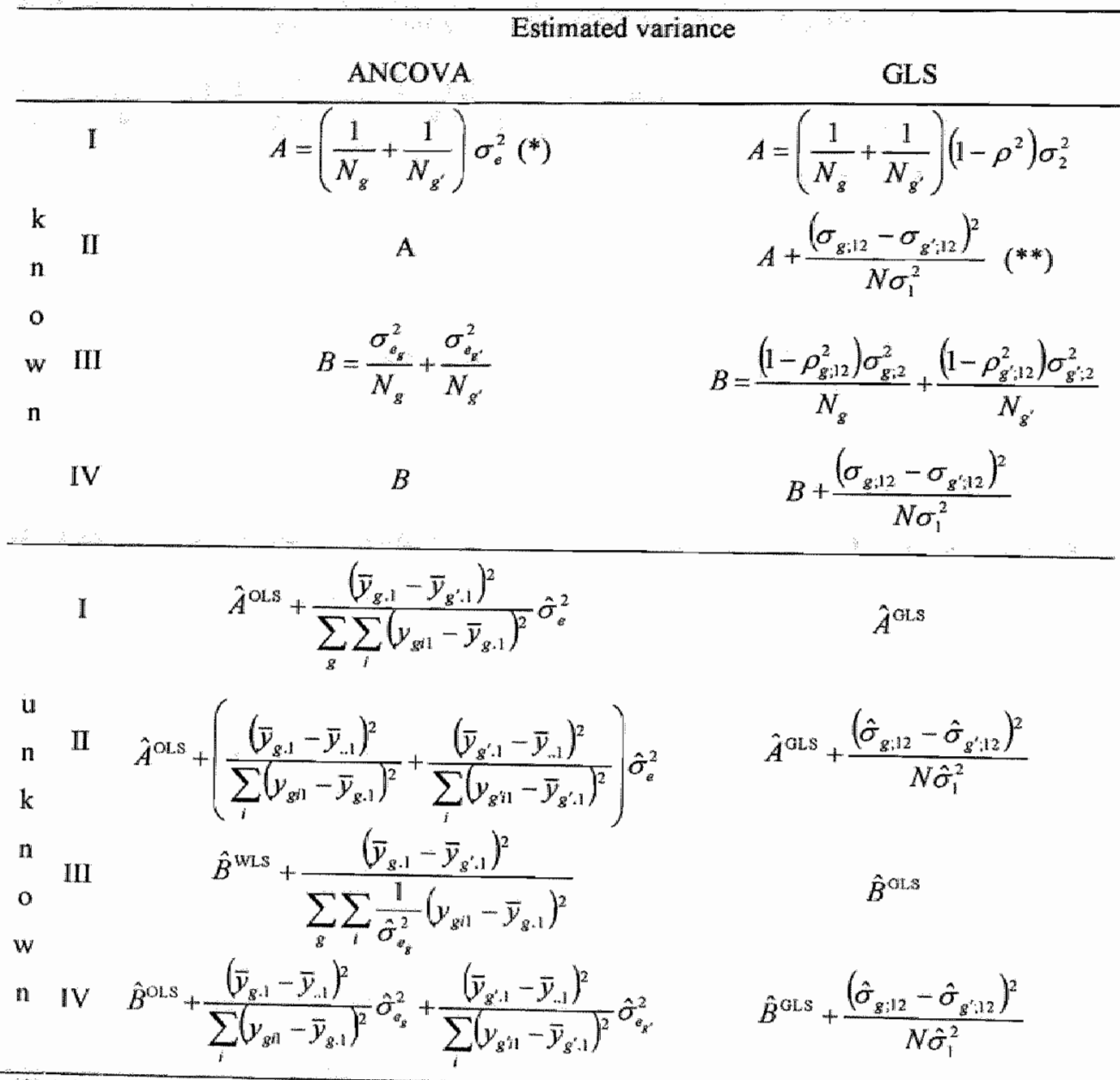

(*) The conditional ( $y_{\text {gn }}$ fixed) variance of the ANCOVA treatment effect estimator is equal to the unconditional variance ( $y_{g i !}$ random), because the conditional variance of $y_{g i 2}-\gamma_{1} y_{g i 1}$ for an arbitrary subject $i$ in group $g$, i.e. $\sigma_{k}{ }^{2}$, is equal to its unconditional variance, i.e. $\sigma_{2}{ }^{2}+y_{1}{ }^{2}$ $\sigma_{1}^{2}-2 \gamma_{1} \sigma_{12}=\left(1-\rho^{2}\right) \sigma_{2}^{2}$

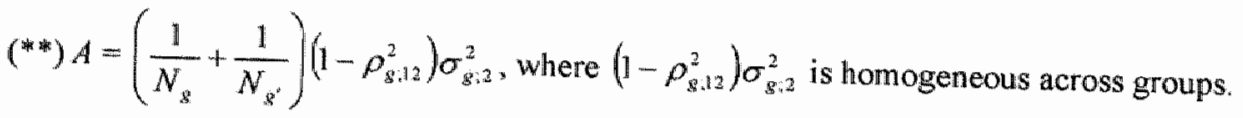




\subsubsection{Situation I: homogeneots slopes and variances}

In practice, the covariance parameters of $y$, here $\sigma_{1}^{2}, \sigma_{12}$ and $\sigma_{2}^{2}$, are unknown and $y=$ $\sigma_{12} / \sigma_{1}{ }^{2}$ and $\sigma_{e}{ }^{2}=\left(1-\rho^{2}\right) \sigma_{2}{ }^{2}$ in Table 2.1 have to be replaced with their unbiased ordinary least squares (OLS) estimators, where $\hat{\sigma}_{a}^{2}$ is the sum of squares of residuals divided by $N$ minus the number of regression parameters, here $G+1$, and

$$
y^{\text {oLs }}=\frac{\sum_{g} \sum_{i}\left(y_{g i 1}-\bar{y}_{g \cdot 1}\right)\left(y_{g i 2}-\bar{y}_{g . z}\right)}{\sum_{g} \sum_{i}\left(y_{g i 1}-\bar{y}_{g, 1}\right)^{2}} .
$$

Due to the replacement of $\gamma$ with its OLS estimator, the variance of the ANCOVA treatment effect estimator will increase (e.g. Armitrage \& Berry, 1987, p. 291). Asymptotically, this variance is equal to the one for known covariance parameters (see Table 2.1).

Homogeneous regression slopes and residual post-treatment variances are obtained in a GLS framework (model (2.3)) by assuming homogeneous covariance matrices, i.e. $V_{1}=\ldots=$ $V_{\mathrm{G}}$. The estimated variance of the GLS treatment effect estimator (Table 2.1) is obtained firom the estimators of $\sigma_{1}{ }^{2}, \sigma_{12}$ and $\sigma_{2}{ }^{2}$, but takes no account of the variability in the estimators of $\sigma_{11}{ }^{2}, \sigma_{12}$ and $\sigma_{2}{ }^{2}$. Therefore, it will generally be biased downwards. An adjustment that is available in current software is discussed in Section 2.3.

\subsubsection{Situation II: heterogeneous slopes and homogeneows variances}

Classically, the non-parallel lines ANCOVA model (2.2) assumes heterogeneous slopes $\gamma_{g}$ and homogeneous residual wariances $\sigma_{2}{ }^{2}$. If $\sigma_{1}{ }^{2}, \sigma_{g ; 1}, \sigma_{g ; 2}{ }^{2}$ and $\mu_{1}$ are unknown, $\mu_{1}$ has to be replaced with the overall pre-treatment mean $\bar{y}_{.1}$ and $\sigma_{e j}{ }^{2}$ by its unbiased OLS estimator, which is pooled over all groups. Moreover, the regression slopes $\gamma_{8}$ have to be replaced with their OLS estimators,

$$
\hat{\gamma}_{g}^{\text {or.s }}=\frac{\sum_{i}\left(y_{g i 1}-\bar{y}_{g \cdot 1}\right)\left(y_{g i 2}-\bar{y}_{g \cdot 2}\right)}{\sum_{i}\left(y_{g i 1}-\bar{y}_{g .1}\right)^{2}} .
$$


As a consequence of replacing $\gamma_{g}$ and $\gamma_{g^{\prime}}$ with their OLS estimates, the variance of the OLS treatment effect estimator will increase (e.g. Maxwell \& Delaney, 2000, p. 414). Asymptotically, this variance is equall to the one for known covariance parameters (Table 2.1). The estimated variance of the OLS treatment effect estimator, however, only corrects for unknown $\gamma_{g}$, but not for unknown $\mu_{1}$. Therefore, for random instead of fixed $y_{g}$, the estimated variance of the OLS treatment effect estimator will generally be biased downwards in case of heterogeneous slopes.

The ANCOVA assumption of heterogeneous regression slopes $y_{g}$ implies heterogeneous covariances $\sigma_{g, 12}$, while homogeneous residual post-treatment variances $\sigma_{e}{ }^{2}$ imply $\left(1-\rho_{g: 12}^{2}\right) \sigma_{g: 2}^{2}=\left(1-\rho_{g^{\prime}: 2}^{2}\right) \sigma_{g^{\prime}: 2}^{2}$. Thus, given $\sigma_{3}^{2}$ homogeneous across groups, due to the randomization, heterogeneous $\gamma_{g}$ and homogeneous $\sigma_{E}{ }^{2}$ can only be obtained in the GLS framework (model (2.3)) by the following constraints, $\sigma_{g ; 12} \neq \sigma_{g}, 12$ and $\sigma_{z: 2}^{2}=\sigma_{g^{\prime} ; 2}^{2}+\left(\sigma_{z: 12}^{2}-\sigma_{g^{\prime}: 12}^{2}\right) / \sigma_{1}^{2}$. These strict constraints show that satisfying homogeneous $\sigma_{e}{ }^{2}$ may be unrealistic in case of heterogeneous $\gamma_{g}$ as was already observed by Aguinis and Pierce (1998) and Larholt and Sampson (1995). The estimated variance of the GLS treatment effect estimator (Table 2.1) is again biased downwards and an adjustment is required (see Section 2.3).

\subsubsection{Simation III: homogeneous slopes and heterogeneous wariances}

We retum to the situation of homogeneous regression slopes $\gamma$ (situation I), but drop the assumption of homogeneous $\sigma_{q}{ }^{2}$. Consequently, WLS should be used instead of OLS (e.g. Dïggle, Liang \& Zeger, 1996). If $\sigma_{1}{ }^{2}, \sigma_{12}$ and $\sigma_{g, 2}{ }^{2}$ are unknown, $\gamma$ and $\sigma_{e_{g}}^{2}$ have to be replaced with their WLS estimators, $\hat{\gamma}^{\text {wh } s}$ and $\hat{\sigma}_{\theta_{g}}^{2}$, where $\hat{\sigma}_{v_{g}}^{2}$ is the usual within-group residual variance estimator and

$$
\hat{\gamma}^{\text {WLS }}=\frac{\sum_{g} \sum_{i}\left(y_{g i 1}-\bar{y}_{g \cdot 1}\right)\left(y_{g i 2}-\bar{y}_{g \cdot 2}\right) / \hat{\sigma}_{v_{g}}^{2}}{\sum_{s} \sum_{i}\left(y_{g i n}-\bar{y}_{g \cdot 1}\right)^{2} / \hat{\sigma}_{e_{g}}^{2}} .
$$


As a consequence of replacing with its WLS estimator, the variance of the WLS treatment effect estimator will increase (see Table 2,1). Note that this variance reduces to the one under homogeneous slopes and residual variances (situation $\mathrm{I}$ ), when $\hat{\sigma}_{\mathrm{e}_{\mathrm{g}}}^{2}$ is the same for all groups.

Homogeneous slopes $\gamma$ imply homogeneous $\sigma_{12,}$ assuming homogeneous $\sigma_{1}{ }^{2}$, and so heterogeneous $\sigma_{e_{i}}^{2}$ then imply heterogeneous $\sigma_{g ; 2^{2}}{ }^{2}$ across groups. If $\sigma_{1}{ }^{2}, \sigma_{12}$ and $\sigma_{g ; 2^{2}}$ are replaced by their estimators, the estimated variance of the GLS treatment effect estimator (Table 2.1) is again biased downwards.

\subsubsection{Sitwation IV: heterogeneous slopes and variances}

In this section, we drop both homogeneity assumptions, i.e. we allow heterogeneous $\gamma_{g}$ and $\sigma_{e_{3}}^{2}$. Model (2.2) can be applied to each group separately to account for these heterogeneities. Consequently, if $\sigma_{11}{ }^{2}, \sigma_{g ; 1}, \sigma_{g ; 2}{ }^{2}$, and $\mu_{1}$ are unknown, OLS estimation can be used to estimate the unknown ANCOVA parameters $\gamma_{g}, \mu_{1}, \mu_{g 2}$ and $\hat{\sigma}_{s_{g}}^{2}$. The OLS estimator of $\gamma_{g}$ is given in equation (2.6) and the OLS estimator of $\mu_{g, 2}$ is $\left(\bar{y}_{g \cdot 2}-\hat{\gamma}_{g}^{\text {OLS }} \bar{y}_{g .1}\right)$. A consequence of unknown $\gamma_{g}$ is that the variance estimator of $\hat{\tau}_{g, g^{*}}^{\text {ols }}$ will increase (see Table 2.1 ; Armitage \& Berry, 1987, p. 153). This variance is still an underestimation for unconditional inference (random $y_{g i 1}$ ), because it does not correct for replacing $\mu_{\|}$by its estimator $\bar{y}_{\ldots 1}$. Asymptotically, this variance is equal to the one for known covariance parameters (Table 2.1).

Another way to deal with heterogeneous $\sigma_{u_{s}}^{2}$ is to model this heterogeneity in a GLS framework. Due to the randomization, $\mu_{1}$ and $\sigma_{1}^{2}$ may be assumed homogeneous, which can lead to a gain in efficiency (e.g. Laird \& Wang, 1990). Heterogeneous $\sigma_{g, 12}$ and $\sigma_{g, 2}{ }^{2}$ are assumed to obtain heterogeneous $\gamma_{g}$ and to impose no restrictions on $\sigma_{e_{j}}^{2}$, respectively. If $\sigma_{1}^{2}$, $\sigma_{g_{*}: 12}, \sigma_{g_{3} 2^{2}}$ are replaced with their estimators, the estimated variance of the GLS treatment effect estimator (Table 2.1) will generally be biased downwards. We will return to this point in the next section, where estimation methods for the unknown covariance components are discussed. 


\section{Chaprer 2}

\subsection{Estimation of unknown covarianee parameters}

The model-based variance estimator of the treatment effect estimator (Table 2.1) assumes that the covariance structure of $V_{g}$ (equation (2.1)) is modelled correctly. A robust or empirical variance estimator, proposed by Liang and Zeger (1986), does not require this assumption to estimate the variance consistently. But this robust estimator is generally biased downwards due to the estimation of the regression parameters which leads to a loss in degrees of freedom and more importantly it is often far more variable than the model-based one (e.g. Kauermann \& Caroll, 2001).

In the previous section, we already indicated that the estimated variance of the GLS treatment effect estimator will generally be biased downwards, because it ignores the variability in the covariance parameter estimators, which are generally obtained by restricted maximum likelihood (REML) (e.g. Verbeke \& Molenberghs, 2000). Kenward and Roger (1997) proposed a variance estimator that adjusts for this bias, but their adjustment is only available for the model-based variance estimator.

\subsection{An example}

The four different ANCOVA and GLS methods will be applied to an example to illustrate the previous results in a realistic setting. In a randomized trial, the protective effect of a single dose of an experimental treatment, formoterol solution aerosol (12 $\mu \mathrm{g})$, is compared to a single dose of a standard therapy, salbutamol suspension aerosol $(100 \mu \mathrm{g})$, and placebo for patients suffering from exercise-induced asthma. The outcome variable, forced expiratory volume in one second $(\mathrm{FEV})$ after an exercise test, was measured for each patient before and two hours after treatment; nine patients received a placebo, ten patients received salbutamol and eleven patients received formoterol. For more information on this trial, we refer to Senn (1993, p. 123-126, 139-140, second period). The mean $F E V_{1}(\mathrm{ml})$ after an exercise test, corresponding standard deviations, covariance and correlation between pre- and post-treatment measures as well as OLS estimates of $\sigma_{w_{g}}^{2}$ and $y_{g}$ which are obtained from applying model (2.2) to each group separately (see situation IV) are given for each group in Table 2.2. 
Table 2.2 Mean FEV, (mi) after an exercise test, corresponding standard deviations, covariance and correlation between pre- and post-treatment measures, residual post-treatment variance and regression slope for each group.

\section{Pre- Post-}

Group Treatment Patients treatment treatment

\begin{tabular}{ccccccccc} 
& & & Mean (SD) & Mean (SD) & $\hat{\sigma}_{g: 12}$ & $\hat{\rho}_{s: 12}$ & $\hat{\sigma}_{e_{s}}^{2}$ & $\hat{\gamma}_{g}^{\text {OLS }}$ \\
\hline 1 & formoterol & 11 & $2127(562)$ & $2429(751)$ & 371517 & 0.88 & 140754 & 1.18 \\
2 & salbutamol & 10 & $2400(744)$ & $2390(528)$ & 273358 & 0.70 & 161727 & 0.49 \\
3 & placebo & 9 & $2256(456)$ & $1678(291)$ & 53911 & 0.41 & 80535 & 0.26 \\
\hline
\end{tabular}

The within-group means and variances of pre-treatment measures were not significantly different, as is expected given randomization. On the other hand, the posttreatment means and variances were significantly different. Table 2.2 suggests heterogeneous slopes and residual variances, but we will see that heterogeneous slopes and homogeneous residual variances fit the data equally well.

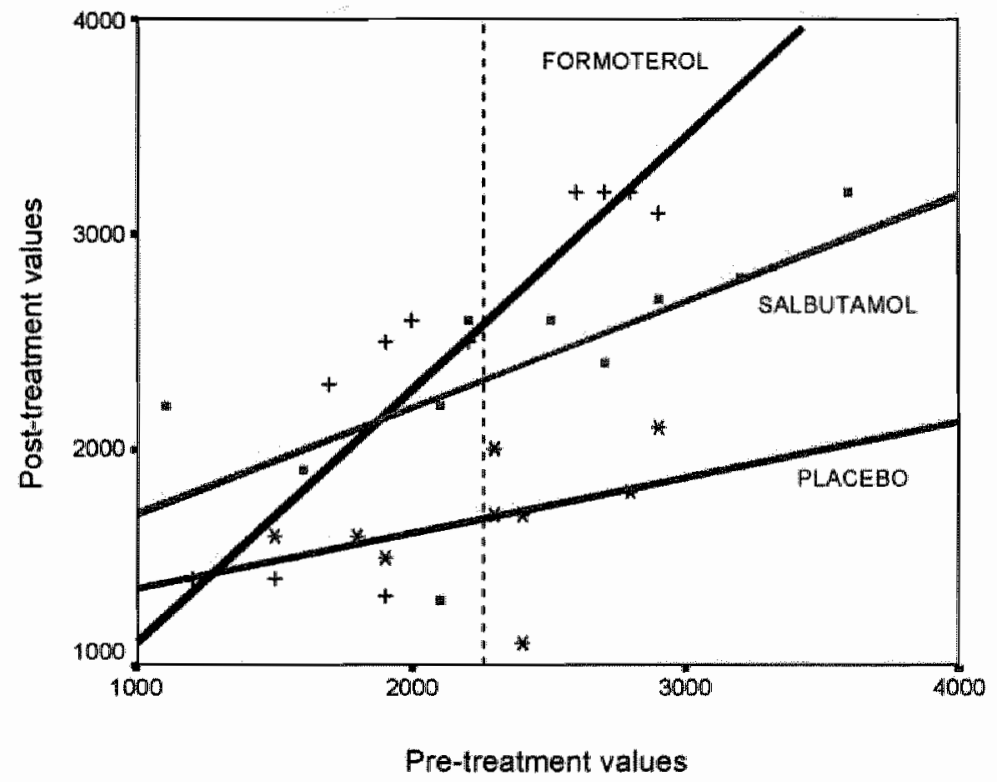

Figure 2.1 $\mathrm{FEV}_{\mathrm{I}}$ post-treatment versus pre-treatment measures: data points and linear regression lines per group. The vertical dashed line represents the overall mean of the pretreatment measures. 


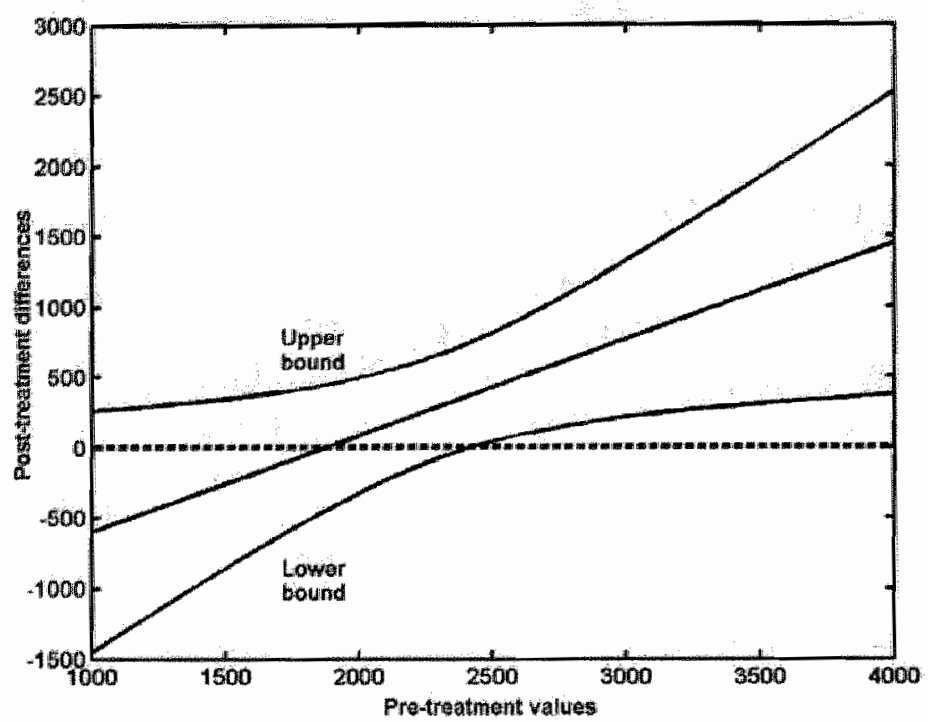

Figure 2.2 Difference between regression lines of formoterol and salbutamol group and confidence bands: $\hat{y}_{k_{2}}-\hat{y}_{s 2} \pm t_{a f, \mathbb{L}-\alpha / 2} \sqrt{S E^{2}\left(\hat{y}_{F_{2}}\right)+S E^{2}\left(\hat{y}_{S^{2} 2}\right)}$, where $\hat{y}_{F_{2}}$ and $\hat{y}_{S 2}$ are predicted post-treatment values of formoterol and salbutamol group, respectively, at a particular pre-treatment value and $d f$ are Satterthwaike degrees of freedom.

Figure 2.1 shows that the placebo group had a smaller regression slope and predicted post-treatment value than the salbutamol group. The formoterol group had a steeper slope than the other groups. This group also had a larger predicted post-treatment value at $\vec{y}_{.1}$ than the other groups (see vertical dashed line). In Figure 2.2, the difference between the regression lines of the formoterol and salbutamol groups and $95 \%$ confidence bands were plotted. Figure 2.2 shows that for subjects whose pre-treatment walue exceeds some threshold (here 2421) formoterol significantly outperformed salbutamol. This shows that a conditional treatment effect is more informative than an overall treatment effect in case of heterogeneous slopes. As for influential subjects, no evidence was found for their existence in this experiment by the Cook's distances.

\subsection{Methods}

Although Table 2.2 suggests heterogeneous slopes $\gamma_{g}$, ANCOVA and GLS models (I and III) that assume homogeneous $\gamma_{g}$ were also applied to the $\mathrm{FEV}_{\mathrm{i}}$ data. The purpose of applying 
these models was to investigate the sensitivity of ANCOVA and GLS estimation to violations of homogeneous $\gamma_{g}$. No GLS model that assumes heterogeneous slopes $\gamma_{g}$ and homogeneous residual variance $\sigma_{e}{ }^{2}$ was applied to the $F E V_{1}$ data, because this required an unusual assumption that could not be modelled (see Section 2.2). The treatment effect estimates and their estimated standard errors (model-based, with and without Kenward and Roger's adjustment, and robust) are presented in Table 2.3, where all models were applied using the SAS (release 8.02) procedure PROC MIXED. The robust standard errors for GLS model IV, however, were obtained by version 1.10.0006 of MLwiN (Rasbash et al., 2000), because SAS gave incorrect robust standard errors (see Appendix for details). The model-based standard errors without Kenward and Roger's adjustment are given in Table 2.3 to show that this adjustment can be substantial for GLS models. The deviances (ML and REML) are also given for each model. The models that were used to obtain the correct covariance structure for GLS models III and IV are given in the Appendix GLS model $V_{3}$, which assumes completely heterogeneous $\boldsymbol{V}_{\mathrm{g}}$, including heterogeneous pre-treatment variance $\sigma_{\mathrm{g}_{*}{ }^{2}}{ }^{2}$, has also been applied to the $\mathrm{FEV}_{1}$ data, because it implies no constraints on $\gamma_{g}$ or $\sigma_{t_{g}}^{2}$ and it is standard available in SAS.

\subsubsection{Results}

The ML deviances of the ANCOVA and GLS models (see Table 2.3) show that: allowing heterogeneous $\gamma_{g}$ improves the model fit significantly, while the REML deviances show that heterogeneity of $\sigma_{e_{g}}^{2}$ hardly affects the model fit. Thus, the models that assume heterogeneous $y_{g}$, i.e. ANCOVA models II and IV as well as GLS models IV and $V$, fit the data well. Furthermore, these models led to smaller (model-based and robust) standard errors than the other models obtained with the same method.

ANCOVA and GLS models IV, i.e. assuming heterogeneous $\gamma_{g}$ and $\sigma_{v_{g}}^{2}$, led to the same treatment effect estimates, but the Kenward and Roger"s adjusted model-based standard errors were smaller for ANCOVA than for GLS. The estimated variance of the ANCOVA treatment effect estimator is biased downwards, in case of heterogeneous regression slopes, because it does not correct for unknown $\mu_{1}$. This bias can be large, especially when the slopes are quite heterogeneous, as is the case when the formoterol and pllacebo groups are compared. In case of homogeneous slopes, $\mu_{1}$ can be omitted from ANCOVA model (2.2) and ANCOVA treatment effect and variance estimators will be unbiased, at least when the parallel-lines 
model is correct. On the other hand, the GLS wariance estimator is biased downwards, because it does not correct for the variability in the covariance parameter estimates. But, as Table 23 shows, in case of homogeneous slopes, GLS and ANCOVA standard errors do not differ much after Kenward and Roger"s adjustment has been applied.

Table 2.3 FEV data. Treatment effect estimates (REML) together with their unadjusted model-based (M), model-based with Kenward and Roger"s (KR) adjustment and robust (R) standard errors for ANCOVA and GLS models. Regression slopes and residual variances are: I - both homogeneous; II - heterogeneous and homogeneous, respectively; III - homogeneous and heterogeneous, respectively; IV - both heterogeneous; $\mathrm{V}$ - completely heterogeneous $V_{g}$. Group 1 - formoterol; 2 - salbutamol; 3 - placebo.

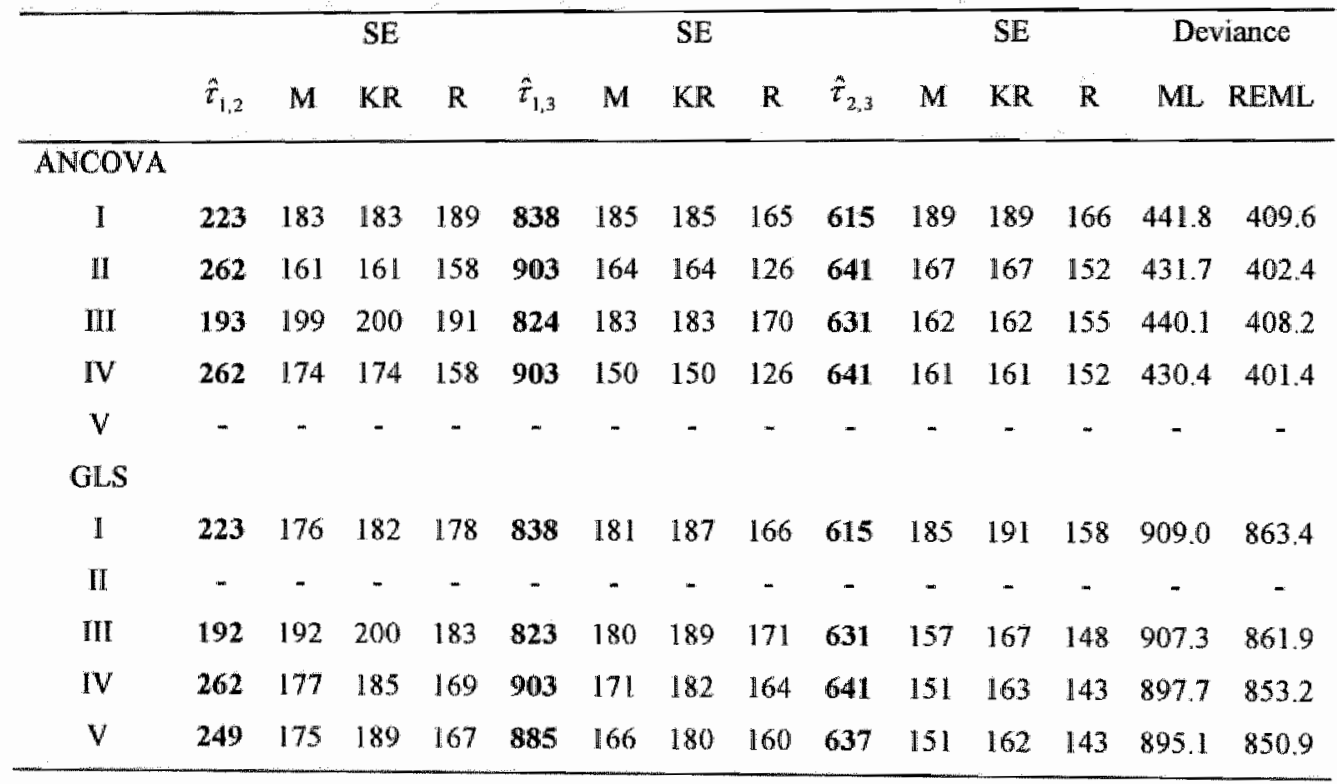

\subsection{Conclusions}

In the present paper. ANCOVA was compared with GLS to estimate an overall treatment effect $\tau_{g, g^{n}}$ under different assumptions about regression slopes $\gamma_{g}$ and residual variances $\sigma_{q_{g}}^{2}$. We found the following results:

I. In case of homogeneous slopes and variances, ANCOVA is preferred to GLS because of unbiased treatment effect and variance estimators. The bias in the variance of the 
GLS treatment effect can, however, be substantially reduced by using Kenward and Roger's adjusted variance estimator.

II. In case of heterogeneous slopes and homogeneous variances, ANCOVA leads to a biased variance estimator of the treatment effect. However, it must be used, because GLS could not be applied with the present software.

III. In case of homogeneous slopes and heterogeneous variances, ANCOVA with WLS instead of OLS estimation is preferred to GLS for the same reason as in case of homogeneous slopes and variances.

IV. In case of heterogeneous slopes and variances, GLS is preferred to ANCOVA, because GLS uses a correction factor for unknown $\mu_{1}$ which is neglected by ANCOVA and can be crucial, especially when the slopes $\gamma_{g}$ are quite heterogeneous. Notice that Kenward and Roger's (1997) adjusted variance estimator has to be used to account for the variability in the covariance parameter estimates.

Unfortunately, Kenward and Roger's adjustment, which was derived for the modelbased variance estimator, can not be used in combination with the robust variance estimator: As a consequence, the robust variance estimator of the GLS treatment effect estimator will be biased downwards due to the variability in the covariance parameter estimators as well as in the regression parameter estimators (see Section 2.3).

Although the present paper discussed overall treatment effects, treatment effects can depend heavily on the pre-treatment value in case of heterogeneous regression slopes (see e.g. Figure 2.2; Fleiss, 1986). Thus, in case of heterogeneous slopes, a conditional treatment effect is more informative than an overall treatment effect, and ANCOVA should be used.

In short, ANCOVA is preferred over the GLS method, because the ANCOVA treatment effect estimators and their variance estimators are unbiased when the regression slopes are homogeneous, and a conditional treatment effect may be more informative than an overall treatment effect in case of heterogeneous slopes. The GLS method, however, can easily be extended to more than two repeated measures, which will be our topic in a future paper, and several methods to deal with missing values are available for GLS methods (e.g. Verbeke \& Molenberghs, 2000, chapters 14-22). 


\section{References}

Aguinis, H., \& Pierce; C. A. (1998). Heterogeneity of error variances and the assessment of moderating effects of categorical variables: A conceptual review, Organizational Research Methods, 1, 296-314.

Armitage, P., \& Berry, G. (1987). Statistical Methods in Medical Research (2nd ed.). Oxford: Blackwell Scientific Publications.

Diggle, J. P., Liang, K-Y, \& Zeger, S. L. (1996). Analysis of Longitudinal Data. Oxford: Clarendon Press.

Fleiss, J. L. (1986). The Design and Analysis of Clinical Experiments. New York: John Wiley \& Sons.

Kauermann, G.s \& Carroll, R. J. (2001). A note on the efficiency of sandwich covariance matrix estimation, Journal of the American Statistical Association, 96, 1387 - 1396.

Kenward, M. G., \& Roger, J. H. (1997). Small sample inference for fixed effects from restricted maximum likelihood, Biomerrics, 53, 983-997.

Laird, N. M., \& Wang, F. (1990). Estimating rates of change in randomized clinical trials, Controlled Clinical Trials, 11, 405-419.

Larholt, K. M., \& Sampson, A. R. (1995). Effects of heteroscedasticity upon certain analyses when regression lines are not parallel, Biometrics, 51, 731 - 737.

Liang, K-Y., \& Zeger, S. L. (1986). Longitudinal data analysis using generalized linear models, Biometrika, 73, 13-22.

Maris, E. (1998). Covariance adjustment versus gain scores - Revisited, Psychological Methods, 3, 309-327.

Maxwell, S. E., \& Delaney, H. D. (2000). Designing Experiments and Analyzing Data: A model comparison perspecrive. Mahwah: Lawrence Erlbaum Associates, Inc.

Rasbash, J., Browne, W., Goldstein, H., Yang, M., Plewis, I., Healy, MI., Woodhouse, G., Draper, D., Langford, I, \& Lewis, T. (2000). A User's Guide to MLwiN. London: Multilevel Models Project, Institute of Education, University of London.

Senn, S. (1993). Cross-over Thials in Clinical Research, Chichester: John Wiley \& Sons Ltd.

Senn, S. (1997). Statistical Issues in Drug Development, Chichester: John Wiley \& Sons Ltd.

Verbeke, G., \& Molenberghs, G. (2000). Linear Mixed Models for Longitudinal Data. New York: Springer. 


\section{Appendix}

Multilevel model used in the $F E V_{1}$ example to specify the correct covariance structure in situation III, i.e. homogeneous $\sigma_{1}{ }^{2}$ and $\sigma_{12}$ and heterogeneous $\sigma_{g_{2} 2^{2}}$.

In this part of the Appendix, a slightly different notation than in the rest of the paper is used to be consistent with the literature about multilevel analysis and the software programs that are used. The multilevel model for the $\mathrm{FEV}_{1}$ data is given by:

$$
\left\{\begin{array}{l}
\boldsymbol{y}_{\boldsymbol{i}}=\boldsymbol{X} \boldsymbol{\beta}+\boldsymbol{Z}_{i} \boldsymbol{b}_{i}+\varepsilon_{i} \\
\boldsymbol{b}_{i} \sim N(\boldsymbol{0}, \boldsymbol{D}) \\
\varepsilon_{i} \sim N(\boldsymbol{0}, \boldsymbol{H}) \\
\boldsymbol{b}_{1}, \ldots, \boldsymbol{b}_{N}, \varepsilon_{1}, \ldots, \boldsymbol{\varepsilon}_{N} \text { independent }
\end{array}\right.
$$

where $y_{i}$ is the 2-dimensional response vector of subject $i_{3} i=1,2, \ldots, N, N$ is the total number of subjects, i.e. $N=N_{\|}+N_{2}+N_{3}=30, X_{t}$ and $Z_{i}$ are matrices of known covariates, $\beta$ and $b_{i}$ are vectors containing the fixed and random effects, respectively, and $\xi_{j}$ is an 2-dimensional vector of residual components. Furthermore, $\boldsymbol{D}$ and $\boldsymbol{H}$ are the level-2 and level-1 covariance matrices, respectively. This multilevel model implies the marginal model

$$
y_{i} \sim N\left(X_{i} \beta_{3} Z_{i} D Z_{i}^{\prime}+\boldsymbol{H}\right)
$$

An option to model the correct covariance structure is to fit the following model,

$$
y_{i j}=\mu_{1}\left(1-t_{j}\right)+\left[\left(\mu_{12}+b_{1 i}\right) G_{1 i}+\left(\mu_{22}+b_{2 i}\right) G_{2 i}+\mu_{32} G_{3 i}\right] l_{j}+\varepsilon_{i j}
$$

where $G_{1 i} G_{2 i}$ and $G_{3 i}$ are group dummies indicating the different groups, i e formoterol, salbutamol and placebo, respectively, and $t_{j}=0,1(j=1,2)$. The corresponding multilevel model for FEV, data implies that the diagonal elements of $\boldsymbol{D}$ are $\operatorname{Var}\left(b_{V i}\right)$ and $\operatorname{Var}\left(b_{2 i}\right)$ and the non-diagonal elements are zero. Furthermore, an unstructured level-1 covariance matrix $\boldsymbol{H}$ is assumed, which is homogeneous across groups. The correct covariance structure is then modelled by these level -1 and level- 2 covariance matrices, since 


$$
\begin{aligned}
\operatorname{Var}\left(y_{i 1}\right) & =\sigma_{x_{1},}^{2}, \\
\operatorname{Var}\left(y_{i 2}\right) & =\left\{\begin{array}{cl}
\operatorname{Var}\left(b_{11}\right)+\sigma_{s_{2}}^{2} & \text { if group 1, } \\
\operatorname{Var}\left(b_{2 i}\right)+\sigma_{\varepsilon_{2}}^{2} & \text { if group 2, } \\
\sigma_{\varepsilon_{3}}^{2} & \text { if group 3, }
\end{array}\right. \\
\operatorname{Cov}\left(y_{i 3}, y_{12}\right) & =\sigma_{s_{12},}
\end{aligned}
$$

where $\sigma_{s_{1}}^{2}, \sigma_{v_{3}}^{2}$ and $\sigma_{\varepsilon_{13}}$ are the error covariance parameters. The group with the smallest $\operatorname{Var}\left(y_{i 2}\right)$, here group 3 , has to be excluded from the level-2 covariance matrix to assure that $\boldsymbol{D}$ is a nonnegative definite matrix. Otherwise, one must allow negative estimates of the covariance parameters, which can cause non-convergence of the procedure. SAS PROC MIXED even requires a positive definite matrix for the robust standard errors to be correct (see SAS note 008648). The random and repeated statements of SAS procedure MDXED for this model are

random timegroup 1 timegroup $2 /$ type $=$ UN subject $=$ ids;

repeated timeclss $/$ type $=$ UN subject $=$ ids.

Multilevel model used in the FEV, example to specify the correct covariance structure in situation IV, i.e. homogeneous $\sigma_{1}^{2}$, heterogeneous $\sigma_{g ; 2}$ and $\sigma_{\mathrm{g} ; 2^{2}}$.

The model (see equation $\mathrm{Al}$ ) for the $\mathrm{FEV}$, data can be written as

$$
y_{i j}=\mu_{1}\left(1-t_{j}\right)+\left[\left(\mu_{12}+b_{1 i}\right) G_{1 i}+\left(\mu_{22}+b_{2 i}\right) G_{2 i}+\left(\mu_{32}+b_{3 i}\right) G_{3 i}\right] t_{j}+\varepsilon_{i j} .
$$

The covariance structure that has to be modelled contains 7 parameters, i.e. $\sigma_{1}^{2}, \sigma_{1,12}, \sigma_{2: 12}$, $\sigma_{3 ; 12}, \sigma_{1: 2}{ }^{2}, \sigma_{2: 2}{ }^{2}$ and $\sigma_{3 ; 2}{ }^{2}$. A result of this multilevel model for FEV, data is that the level-2 covariance matrix $D$ is a $4 \times 4$ covariance matrix, where $\operatorname{Cov}\left(b_{11}, b_{2 i}\right)=\operatorname{Cov}\left(b_{1 i}, b_{3 i}\right)=\operatorname{Cov}\left(b_{2 i}\right.$, $\left.b_{3 j}\right)=0$, since a subject can only belong to one treatment group. Thus, $D$ contains already 7 covariance parameters, which means that the level-1 covariance parameters have to be fixed. The correct covariance structure is now modelled by this level-2 covariance matrix $\boldsymbol{D}$ and an independence level-1 covariance structure, i.e. $\boldsymbol{H}=\sigma_{s}^{2} I$, where $\sigma_{\sigma}^{2}$ is fixed at a small positive value, 


$$
\begin{aligned}
\operatorname{Var}\left(y_{i 1}\right) & =\operatorname{Var}\left(b_{0 i}\right)+\sigma_{s}^{2}, \\
\operatorname{Var}\left(y_{i 2}\right) & =\operatorname{Var}\left(b_{i j}\right)+\sigma_{s}^{2} \text { for group } g(=1,2,3), \\
\operatorname{Cov}\left(y_{i 1}, y_{i 2}\right) & =\operatorname{Cov}\left(b_{0 i}, b_{g i}\right) \text { for group } g(=1,2,3) .
\end{aligned}
$$

The robust standard errors of the treatment effect estimators in contrast to the model-based ones, depended on the fixed value of $\sigma_{\varepsilon}^{2}$ in SAS, whereas this level-1 variance $\sigma_{\varepsilon}^{2}$ is automatically fixed at 0 in MLwiN. SAS note 008648 indicates that the problem in SAS was caused by a bug in the program. This problem can be avoided by allowing all covariance parameters, i.e. $\sigma_{\mathrm{g}: 1}{ }^{2}, \sigma_{\mathrm{g}: 12}, \sigma_{\mathrm{g}: 2}{ }^{2}$, to be heterogeneous (GLS model V) "This covariance structure is modelled by heterogeneous unstructured level- 1 covariance matrices $\boldsymbol{H}_{g}$ with no random effects, i.e. $D=0$. The random and repeated statements of SAS procedure MIXED for this model are

random time0 timegroup1 timegroup2 timegroup3 $/$ type $=$ UN subject $=\mathrm{idls}$; repeated timeclss $/$ type $=$ simple subject $=$ ids. 
Chapter 2 
Log-transformation and power

Log-transformation and power 


\title{
Choprer 3
}

\begin{abstract}
In this paper, we assume that the data of two or three equally large treatment groups are lognormally distributed with equal within-group (residual) variances after log-transformation. This paper shows that, in case of two treatment groups, the gain in efficiency by applying the two-sample /-test, analysis of variance (ANOVA) or analysis of covariance (ANCOVA) to log-transformed instead of untransformed data can be substantial if the coefficient of variation of the untransformed data is large. In case of three groups, the same pattern holds. An example of duration of stay in hospital after anaesthesia is used to illustrate the advantages of log-transforming the data before using ANOVA and two-sample f-tests in a realistic setting.
\end{abstract}

Keywords: Analysis of covariance (ANCOVA); Analysis of variance (ANOVA); Asymptotic relative efficiency (ARE); Log-normal; Transformation; Two-samplle $t$-test; 


\subsection{Introduction}

The two-sample $t$-test assumes that the outcome variable of interest is normally distributed. If this assumption is violated, the two-sample $t$-test may still have good control over the probability of a type I error, depending on sample size and severity of the violations. At the same time, however, this $t$-test can have poor power and be biased, i.e. the power can actually decrease as we move away from the null hypothesis of equal means (e.g. Wilcox, 2001). For positively skewed data, a log-transformation can often be used to normalize the distribution (e.g. Keene, 1995). In the present paper, we assume that the data of equally large treatment groups are independent and log-normally distributed with equal within-group variances after log-transformation. This equality of variances implies that the coefficient of variation (CV), i.e. standard deviation divided by the mean, of the untransformed data is homogeneous across the groups. In other words, the standard deviation of the untransformed data is proportional to the mean. If the $\mathrm{CV}$ of the untransformed data is not homogeneous across groups, then the null hypotheses of the untransformed and log-transformed data are not equivalent. In that case, likelihood based methods, like the modified signed log-likelihood test, which was introduced by Barndorff-Nielsen $(1986 ; 1991)$, perform well in terms of power and confidence level (Wu et al., 2001).

The purpose of the present paper is to show that frequently used methods, like the twosample t-test, analysis of variance (ANOVA) and analysis of covariance (ANCOVA), should be applied to log-transformed instead of untransformed data in case the data are positively skewed and the standard deviation is proportional to the mean, i.e. the $\mathrm{CV}$ is homogeneous across groups. Until Section 3.4, it is assumed that there are only two treatment groups. The asymptotic relative efficiency (ARE) of the two-sample $t$-test based on untransformed versus log-transformed data is described in Section 3.2. In Section 3.3, ANCOVA of a posttreatment measurement with the pre-treatment measurement as a covariate, is discussed. The natural logarithms of the pre- $\left(y_{1}\right)$ and post-treatment measures $\left(y_{2}\right)$ are assumed to follow a bivariate normal distribution with a homogeneous covariance matrix across groups. The ARE of ANCOVA applied to untransformed versus log-transformed data is then derived. In Section 3.4, the ARE of ANOVA applied to untransformed versus log-transformed data is discussed for three treatment groups. In Section 3.5, ANOVA and two-sample 1-tests are applied to duration of stay in hospital after anaesthesia to illustrate the benefits of logtransforming the data before using ANOVA and two-sample t-tests in a realistic setting. We 


\section{Chapter 3}

end this paper with some conclusions and discussion about the asymptotic relative efficiencies of the two-sanple 1 -tests, ANOVA and ANCOVA.

\subsection{Two-sample t-test}

Let $y_{g i 2}$ be the outcome variable (post-treatment measure) of the ith subject in group $g$ $(l=1,2, \ldots, g=1,2)$ in a randomized study with two equally large treatment groups. Assume that the natural logarithms of $y_{12}$ and $y_{22}$ are independently and normally distributed with means $\mu_{12}$ and $\mu_{22}$, respectively, and equal variances, i.e. $\sigma_{12}{ }^{2}=\sigma_{22}{ }^{2}=\sigma_{2}{ }^{2}$. The mean and variance of the untransformed data of group $g$ are then

$$
M_{g 2}=\exp \left(\mu_{g^{2}}+\sigma_{2}^{2} / 2\right) \text { and } V_{g 2}=\exp \left(2 \mu_{g 2}+\sigma_{2}^{2}\right)\left\{\exp \left(\sigma_{2}^{2}\right)-\mathbb{1}\right\}
$$

respectively (e.g. Everitt, 1998). Note that if $\sigma_{12}{ }^{2} \neq \sigma_{22}{ }^{2}$, the null hypothesis for untransformed data, i.e $\mathrm{H}_{0}: M_{12}=M_{22}$, is not equivalent to the null hypothesis for log-transformed data, i.e. $\mathrm{H}_{0}{ }^{*}: \mu_{12}=\mu_{22}$. Under $\boldsymbol{H}_{0}{ }^{*}$,

$$
\begin{aligned}
M_{12}-M_{22} & =\exp \left(\mu_{12}+0.5 \sigma_{12}{ }^{2}\right)-\exp \left(\mu_{22}+0.5 \sigma_{22}{ }^{2}\right) \\
& =\exp \left(\mu_{12}\right)\left\{\exp \left(0.5 \sigma_{12}{ }^{2}\right)-\exp \left(0.5 \sigma_{22}{ }^{2}\right)\right\}
\end{aligned}
$$

which is equal to zero if and only if $\sigma_{12}{ }^{2}=\sigma_{22}{ }^{2}$.

The relative efficiency of the two-sample $t$-test based on untransformed versus logtransformed data is defined as the inverse ratio of the sample sizes required by these $t$-tests to achieve the same power (e.g. Everitt, 1998). We assume for convenience that sample sizes are sufficiently large for applying the normal approximation to the $t$-distribution, in case of untransformed as well as log-transformed data. As a consequence,

$$
\mathrm{ARE}=\frac{n_{1 \mathrm{og}}}{n_{\mathrm{un}}}=\frac{2 \sigma_{2}^{2}\left(M_{12}-M_{22}\right)^{2}}{\left(\mu_{12}-\mu_{22}\right)^{2}\left(V_{12}+V_{22}\right)}
$$

where $n_{\log }$ and $n_{u n i}$ are the sample sizes required by the two-sample $t$-test on log-transformed and untransformed data, respectively (e.g. Schouten, 1999). This asymptotic relative efficiency (ARE) can also be expressed in terms of the coefficient of variation of the 
untransformed post-treatment data $y_{2}$, i.e. $\mathrm{CV}_{2}=\sqrt{V_{2}} / M_{3}=\sqrt{\exp \left(\sigma_{2}^{2}\right)-1}$, which is assumed homogeneous across groups, and the standardized treatment effect $d=\left(\mu_{12}-\mu_{23}\right) / \sigma_{2}$ of the logtransformed post-treatment data,

$$
\mathrm{ARE}=\frac{2\left\{1-\exp \left(-d \sqrt{\ln \left(\mathrm{CV}_{2}^{2}+1\right)}\right)\right\}^{2}}{d^{2} \mathrm{CV}_{2}^{2}\left\{1+\exp \left(-2 d \sqrt{\ln \left(\mathrm{CV}_{2}^{2}+1\right)}\right)\right\}}
$$

Since the $\mathrm{CV}_{2}$ is homogeneous across groups, it is a useful measure of variability in the untransformed data. In practice, for log-normal data the $\mathrm{CV}_{2}$ can be as large as 1 or even larger. For example, Chelmow et al. [8] conducted a study, in which one of the gaals was to estimate the average total costs of neonatal care for each of the 19 triplet pregnancies, and found $\mathrm{a} \mathrm{CV}_{2}=1.2$.

The standardized treatment effect or Cohen's $d=\left(\mu_{12}-\mu_{22}\right) / \sigma_{2}$ is commonly used in quantitative reviews. According to Cohen (1988), standardized treatment effects can be roughly categorized as small $(d=0.2)$, medium $(d=0.5)$ or large $(d=0.8)$. Figure 3.1 shows the ARE against Cohen's $d$ with curves for different values of $C_{2}$.

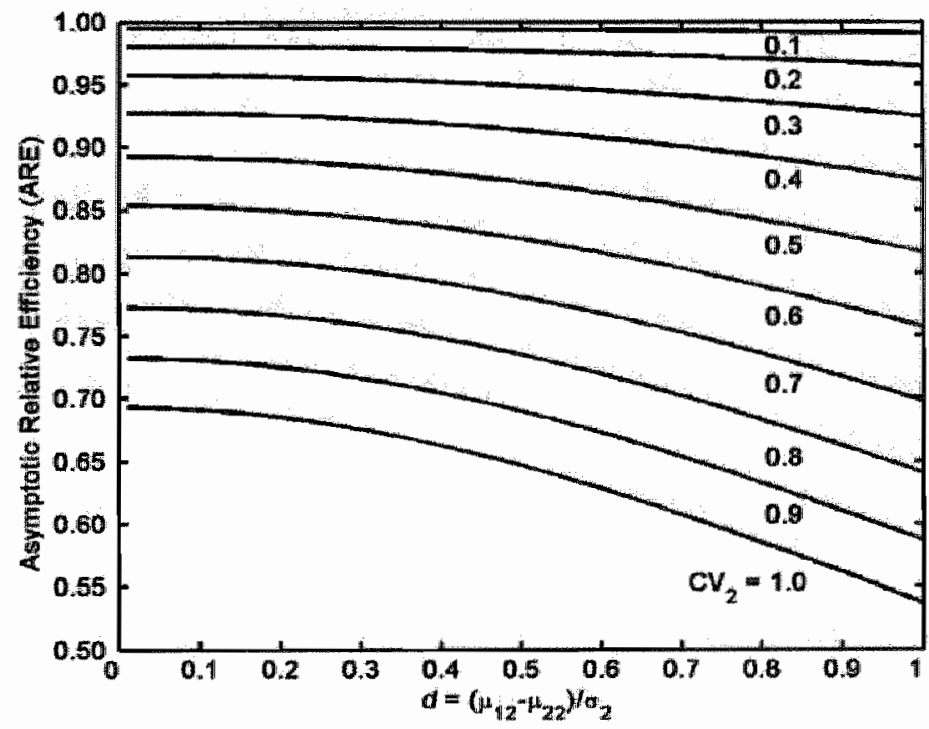

Figure 3.1 ARE of two-sample $t$-test on untransformed versus log-transformed data. 
Figure 3.1 shows that for log-mormal data with homogeneous $\mathrm{CV}_{2}$ the $t$-test on logtransformed data may be much more efficient than the -test on untransformed data. The ARE

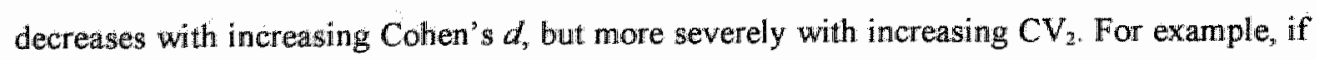
$\mathrm{CV}_{2}$ increases from 0.5 to $1,0, \mathrm{ARE}$ decreases from 0.9 to 0.7 for $d=0.1$ and from 0.82 to 0.54 for $d=1.0$.

\subsection{Analysis of covariance}

Consider a randomized study with two treatments and a quantitative outcome variable. In group $g$, subject $/$ has a pre-treatment measure $y_{g^{i}}$ and a post-treatment measure $y_{g i 2} ; g=1$, 2 and $j=1,2, \ldots, n$. Assume that the natural logarithms of $y_{g i 1}$ and $y_{g i 2}$ follow a bivariate normal distribution with mean vector $\left(\mu_{1}, \mu_{\mathrm{g} 2}\right)^{\prime}$ and homogeneous within-group 2 by 2 covariance matrix, i.e.

$$
\left(\begin{array}{l}
\log \left(y_{g i 1}\right) \\
\log \left(y_{g i 2}\right)
\end{array}\right) \sim N\left(\left(\begin{array}{c}
\mu_{1} \\
\mu_{g^{2}}
\end{array}\right),\left(\begin{array}{cc}
\sigma_{1}^{2} & \rho \sigma_{1} \sigma_{2} \\
\rho \sigma_{1} \sigma_{2} & \sigma_{2}^{2}
\end{array}\right)\right)
$$

where $\mu_{4,}, \sigma_{1}$ and $\mu_{g 2}, \sigma_{2}$ are the pre- and post-treatment means and standard deviations after the log-transformation, respectively, and $\rho$ is the correlation between pre- and post-treatment measures after log-transformation. The homogeneity of the covariance matrix implies that the coefficients of variation of $y_{g n}$ and $y_{g i 2}$ are homogeneous across the groups. Note that the mean and variance of $y_{g i 1}$, i.e. $M_{1}=\exp \left(\mu_{1}+\sigma_{1}{ }^{3} / 2\right)$ and $V_{1}=\exp \left(2 \mu_{1}+\sigma_{1}{ }^{3}\right)\left\{\exp \left(\sigma_{1}{ }^{2}\right)-1\right\}$, respectively, are both homogeneous across groups as a consequence of randomization. On the other hand, the mean and variance of $y_{g / 2}$ may be influenced by the treatments, indicated by subscript $g$ (see equation (3.1)). The correlation $R$ between $y_{g i}$ and $y_{g i 2}$ is homogeneous across groups and $R \leq \rho$ (see Appendix 1 for derivation of the correllation $R$ and Appendix 2 for a sketched proof of $R \leq \rho$ ). with mean

The conditional distribution of $\log \left(y_{g i}\right)$, given $\log \left(y_{g i 1}\right)=\log \left(y_{1}\right)$ or $y_{g i}=y_{1}$, is normal

$$
\mu_{s 2}+\rho \frac{\sigma_{2}}{\sigma_{1}}\left\{\log \left(y_{1}\right)-\mu_{1}\right\}
$$




\section{Log-Iransformation and power}

and variance $\left(1-\rho^{2}\right) \sigma_{2}{ }^{2}$. This implies that $y_{g i 2}$ is, conditional on $y_{g i 1}=y_{1}, \log$-normally distributed with mean

$$
\exp \left[\mu_{g 2}+\rho \frac{\sigma_{2}}{\sigma_{1}}\left\{\log \left(y_{1}\right)-\mu_{1}\right\}+\left(1-\rho^{2}\right) \sigma_{2}^{2} / 2\right]
$$

and variance

$$
\exp \left[2 \mu_{g 2}+2 \rho \frac{\sigma_{2}}{\sigma_{1}}\left\{\log \left(y_{1}\right)-\mu_{1}\right\}+\left(1-\rho^{2}\right) \sigma_{2}^{2}\right] \times\left[\exp \left\{\left(1-\rho^{2}\right) \sigma_{2}^{2}\right\}-1\right]
$$

This conditional mean (3.6) can be written as $\mathrm{a}_{\mathrm{g}} y_{1}{ }^{b}$, where $\mathrm{b}=\rho \sigma_{2} / \sigma_{\mathrm{b}}$ and

$$
\mathrm{a}_{\mathrm{s}}=\exp \left\{\mu_{g^{2}}-\mathrm{b} \mu_{1}+\left(1-\rho^{2}\right) \sigma_{2}^{2} / 2\right\}
$$

This mean (3.6) is a non-linear function of $y_{1}$ if $b \neq 1$, but the linearity assumption of ANCOVA can be approximately walid for a particular range of pre-treatment values. Further, the regression slopes, $R \sqrt{V_{g 2}^{*} / V_{1}}$, and residual variances, $\left(1-R^{2}\right) V_{g 2}^{*}$, are still heterogeneous across groups for untransformed data. Therefore, the assumption of parallelism and homogeneity of error variances will be violated for the untransformed data if the logarithms of $y_{g i 1}$ and $y_{g / 2}$ follow a bivariate normal distribution with a homogeneous covariance matrix.

The ARE of ANCOVA applied to untransformed versus log-transformed data is derived from the ARE of the two-sample t-test, i.e. equation $(3,2)$, by replacing the posttreatment variances $\sigma_{2}{ }^{2}, V_{12}$ and $V_{22}$ with the residual post-treatment variances, $\left(1-\rho^{2}\right) \sigma_{2}{ }^{2}$, $\left(1-R^{2}\right) V_{12}$ and $\left(1-R^{2}\right) V_{22}$, respectively (e.g. Fleiss, 1986). Thus,

$$
\mathrm{ARE}_{\mathrm{ANCONA}}=\frac{2 \sigma_{2}^{2}\left(M_{12}-M_{22}\right)^{2}}{\left(\mu_{12}-\mu_{22}\right)^{2}\left(V_{12}+V_{22}\right)} \times \frac{\left(1-\rho^{2}\right)}{\left(1-R^{2}\right)}
$$

where

$$
R^{2}=\frac{\left\{\exp \left(\rho \sigma_{1} \sigma_{2}\right)-1\right\}^{2}}{\left\{\exp \left(\sigma_{1}^{2}\right)-1\right\}\left\{\exp \left(\sigma_{2}^{2}\right)-1\right\}}
$$




\section{Chapter 3}

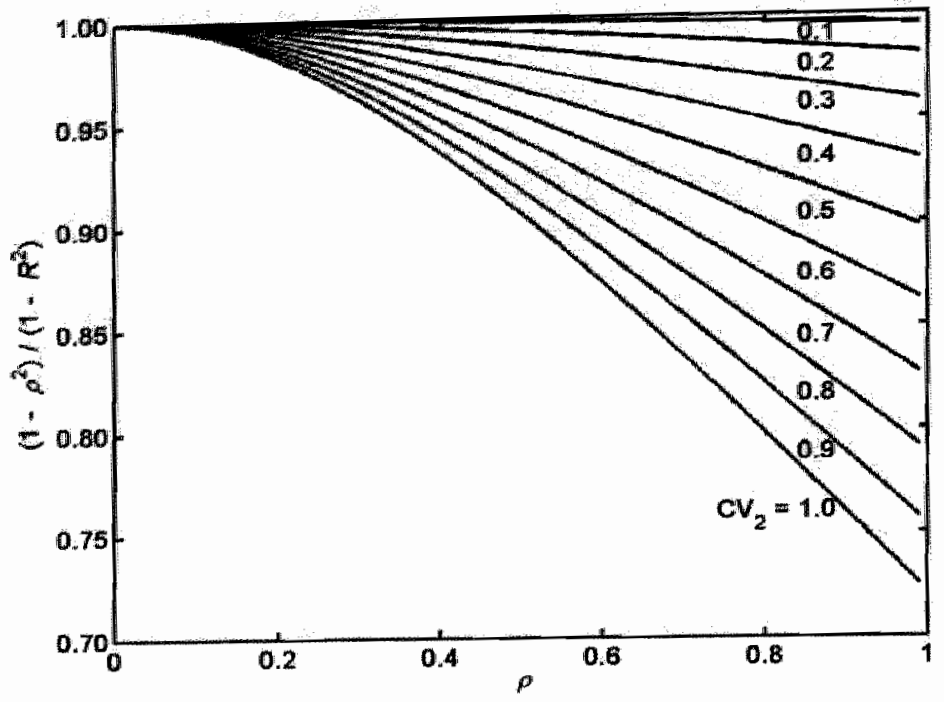

Figure 3.2 The factor $\left(1-\rho^{2}\right) /\left(1-R^{2}\right)$ of the $\operatorname{ARE}_{A N C O N_{A}}$ in equation (3.9).

Note that ARE ANCovd, equation (3.9), is equal to the ARE in equation (3.2), multiplied by the factor $\left(1-\rho^{2}\right) /\left(1-R^{2}\right)$, which is plotted versus $\rho$ with different curves for different values of $\mathrm{CV}_{2}$ of the untransformed post-treatment data in Figure 3.2. This factor decreases with increasing $\rho$ and $\mathrm{CV}_{2}$. If $\rho>0.5$ and $\mathrm{CV}_{2}>1.0$, then $\left(1-\rho^{2}\right) /\left(1-R^{2}\right)<0.9$, which means that ARE of ANCOVA $<0.9 \times$ ARE of two-sample $t$-test on untransformed versus log-transformed data. For convenience, the coefficient of variance is also assumed homogeneous across time points in Figure 3.2 , i.e. $\mathrm{CV}_{1}=\mathrm{CV}_{2}$. If the coefficient of variance is assumed homogeneous across groups, but heterogeneous across time points, the factor $\left(1-p^{2}\right) /\left(1-R^{2}\right)$ is still $\leq 1$ (for proof, see Appendix 2) and it decreases with increasing $\mathrm{CV}_{1}$, $C V_{2}$ and $\rho_{0}$ where $C V_{1}=\sqrt{ }\left\{\exp \left(\sigma_{1}^{2}\right)-1\right\}$ and $C V_{2}=\sqrt{\left\{\exp \left(\sigma_{2}^{2}\right)-1\right\}}$

For the ARE in equation (3.9), ANCOVA must be applied to untransformed data correctly, that is, assuming that the regression slopes and residual variances are heterogeneous across groups. If ANCOVA is applied to the untransformed data as if we had homogeneous instead of heterogeneous slopes and residual variances, then the heterogeneous residual variances $\left(1-R^{2}\right) V_{12}$ and $\left(1-R^{2}\right) V_{22}$ are replaced with the pooled residual variance $V_{a *}$

$$
V_{s}=\frac{V_{12}+V_{22}}{2}\left(1-R^{2}\right)+\frac{R^{2}}{4}\left(\sqrt{V_{12}}-\sqrt{V_{22}}\right)^{2}
$$


(the derivation of $V_{\varepsilon}$ is given in Appendix 3). This residual variance $V_{\delta}$ is larger than the mean of the heterogeneous residual variances, i.e $\left(1-R^{2}\right)\left(V_{12}+V_{22}\right) / 2$. As a consequence, the ARE of ANCOVA, i.e. equation (3.9), decreases even more.

In short, if the logarithms of $y_{g i}$ and $y_{g i 2}$ follow approximately a bivariate normal distribution with a homogeneous covariance matrix, i.e. homogeneous $\mathrm{CV}^{\prime}$ 's of $y_{g i !}\left(\mathrm{CV}_{1}\right)$ and $y_{g n}\left(\mathrm{CV}_{2}\right)$ across groups, one gains efficiency by applying ANCOVA to log-transformed instead of untransformed data. This gain in efficiency increases with increasing $C V_{1}$ and/or $\mathrm{CV}_{2}$ of the untransformed data, increasing standardized treatment effect $d$ of the logtransformed data and increasing correlation $\rho$ between $\log \left(y_{g i}\right)$ and $\log \left(y_{g i 2}\right)$. For example, if $\mathrm{CV}_{2} \geq 1.0$ and $d \geq 0.5$, then ARE of two-sample $t$-test $\leq 0.65$ (see Figure 3.1). In addition, if $\mathrm{CV}_{1}=\mathrm{CV}_{2}$ and $\rho \geq 0.5$, then $\left(1-\rho^{2}\right) /\left(1-R^{2}\right) \leq 0.93$ (see Figure 3.2) and ARE ANCONA $\leq 0.65$ $\times 0.93=0.60$. This means that the required sample size is then reduced with $40 \%$ by applying ANCOVA to the log-transformed instead of the untransformed data.

\subsection{Analysis of variance for more than two groups}

The logarithms of the post-treatment measures $y_{g i 2}(i=1,2, \ldots, n, g=1,2, \ldots, G)$ are assumed to be independently and normally distributed with means $\mu_{g 2}$ and variances $\sigma_{g 2}{ }^{2}=\sigma_{2}{ }^{2}$. Therefore, the ANOVA $F$-test, which assumes homogeneous within-group variances, can be used to compare the means of the log-transformed data. For the untransformed data, the variances $V_{g 2}$ are heterogeneous across groups (see equation $(3.1)$ ) and the Welch (1951) test, which assumes heterogeneous within-group variances, can be used. As a consequence, the ARE of ANOVA applied to untransformed versus log-transformed data is

$$
\operatorname{ARE}_{\mathrm{ANCNA}}=\frac{m_{\mathrm{log}}}{n_{\mathrm{lim}}}=\frac{\sum_{g}\left(M_{g^{2}}-\bar{M}_{w}\right)^{2} / V_{z^{2}}}{\sum\left(\mu_{g^{2}}-\bar{\mu}_{2}\right)^{2} / \sigma_{2}^{2}},
$$

where $\bar{M}_{w}$ is the weighted mean of the untransformed data with weights equal to the inverse of the variances (for derivation of $\mathrm{ARE}_{\mathrm{ANowA}}$, see Appendix 4). For $G=2$, this $\mathrm{ARE}$ reduces to the ARE of the two-sample $t$-test (equation (3.2)). As for $G=3$, one can show that the ARE $_{\text {ANOVA }}$ reduces to 


$$
\mathrm{ARE}_{\text {AWWA }}=\frac{3 \sigma_{2}^{2}}{\exp \left(\sigma_{2}^{2}\right)-1} \times\left\{\frac{1-\frac{1+\exp \left(d_{31}\right)+\exp \left(d_{31}\right)}{\exp \left(d_{21}-d_{31}\right)+\exp \left(d_{31}-d_{21}\right)+\exp \left(d_{31}+d_{31}\right)}}{d_{21}^{2}+d_{31}^{2}-d_{21} d_{31}}\right\}
$$

where $d_{g g^{\prime}}=\left(\mu_{g^{2}}-\mu_{z^{\prime 2}}\right) / \sigma_{2}$ is the standardized treatment effect between groups $g$ and $g^{\prime}$. In

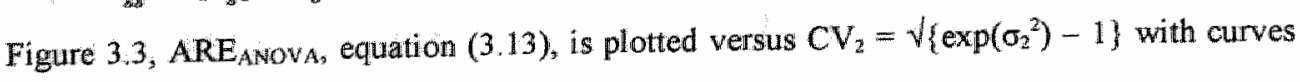
for $d_{31} / d_{21}=1,2$ and 3 , assuming $d_{21}=0.2$.

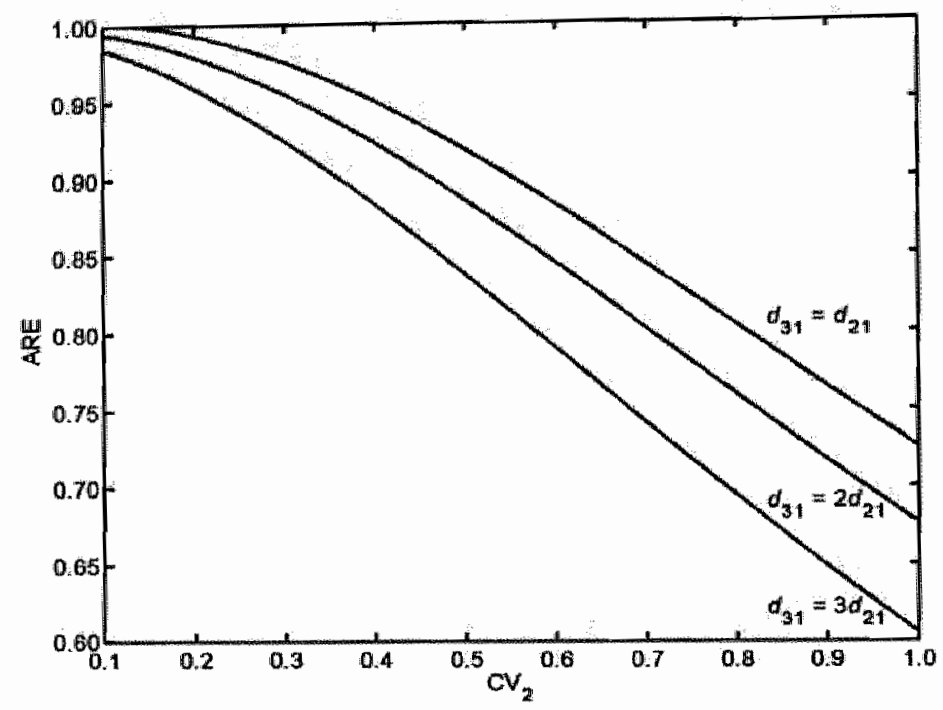

Figure 3.3 ARE ANova versus $C V_{2}$ of the untransformed data with $d_{21}=\left(\mu_{21}-\mu_{12}\right) / \sigma_{2}=0.2$.

For $d_{3}=-0.8,-0.5,-0.2,0.5,0.8$ (not shown here), we saw the same pattern as in Figure 3.3 , i.e. the ARE decreases from 1.0 to roughly 0.7 when $\mathrm{CV}_{2}$ increases from 0.1 to 1.0. In case of four or more groups, the AREANOva is not easilly plotted, since it involves many more parameters.

\subsection{Example}

In a randomized, controlled trial (Myles ef al, 2000), the rate and quality of recovery were compared for four standard anaesthetic regimens used in adults undergoing a range of 
inpatient surgical procedures. One of the outcome variables was the duration of stay in hospital (in days) after anaesthesia. The patients were randomly allocated to one of four anaesthetic regimens (thiopental-isoflurane, propofoll-isoflurane, propofol induction and maintenance or sevoflurane induction and maintenance).

GROUP 1

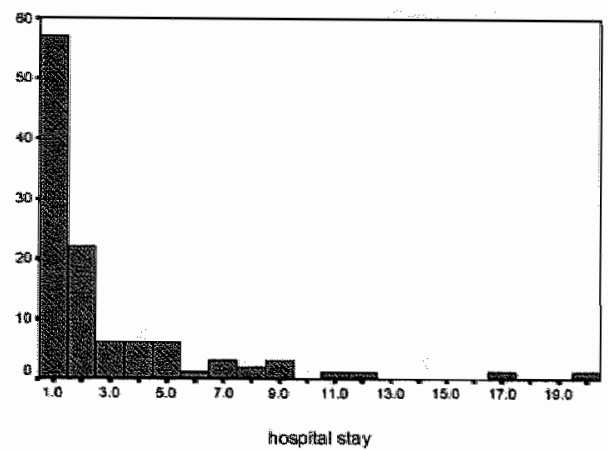

GROUP 3

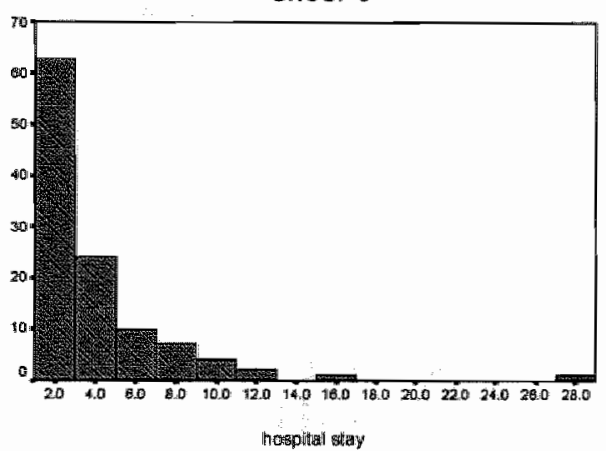

GROUP 2

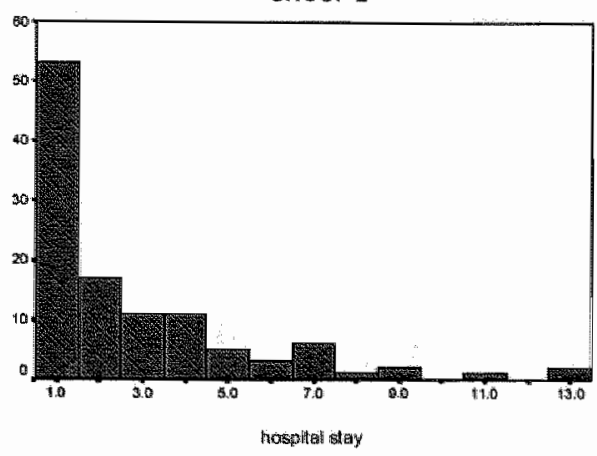

GROUP 4

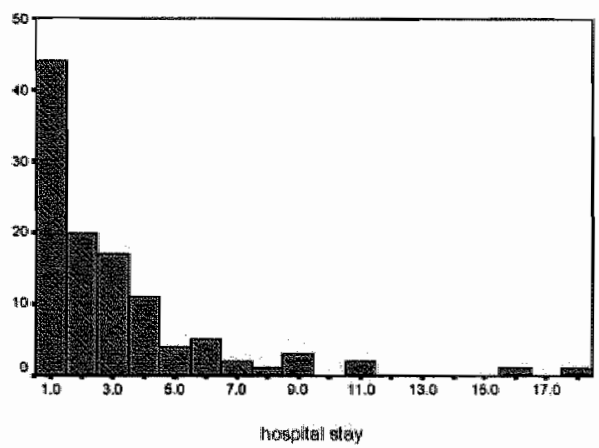

Figure 3.4 Histograms of duration of stay in hospital.

In the four treatment groups $(n=110,112,112$ and 111 , respectively), the mean (SD) durations of hospital stay were $2.92(3.21), 2.97(2.60), 3.59(3.61)$ and 3.20 (2.96) days. The histograms of duration of hospital stay showed a positively skewed distribution and outliers (see Figure 3.4). After log-transformation, the mean (SD) duration of hospital stay in the treatment groups were $0.73(0.74), 0.81(0.71), 0.95(0.78)$ and $0.88(0.71)$, respectively. The log-transformation reduced the coefficient of variation, the skewness and the outliers, but it did not normalize the data (see Figure 3.5). Another log-transformation, i.e. $\log (1+\log ($ hospital stay $)\}$, was also tried, because the log-transformed data still show a positive skew and have a large coefficient of variation. Although the coefficient of variation, 
skewness and outliers were then further reduced and $p$-values of the ANOVA $F$-test and twosample t-tests, not shown here, approached those of the non-parametric methods, the data were not normalized. Non-parametric methods, like the Kruskal-Wallis and Mann-Whitney tests, may have greater power and a higher confidence level than parametric tests in case of non-normal distributions, but non-parametric tests are not easily extended with covariates.
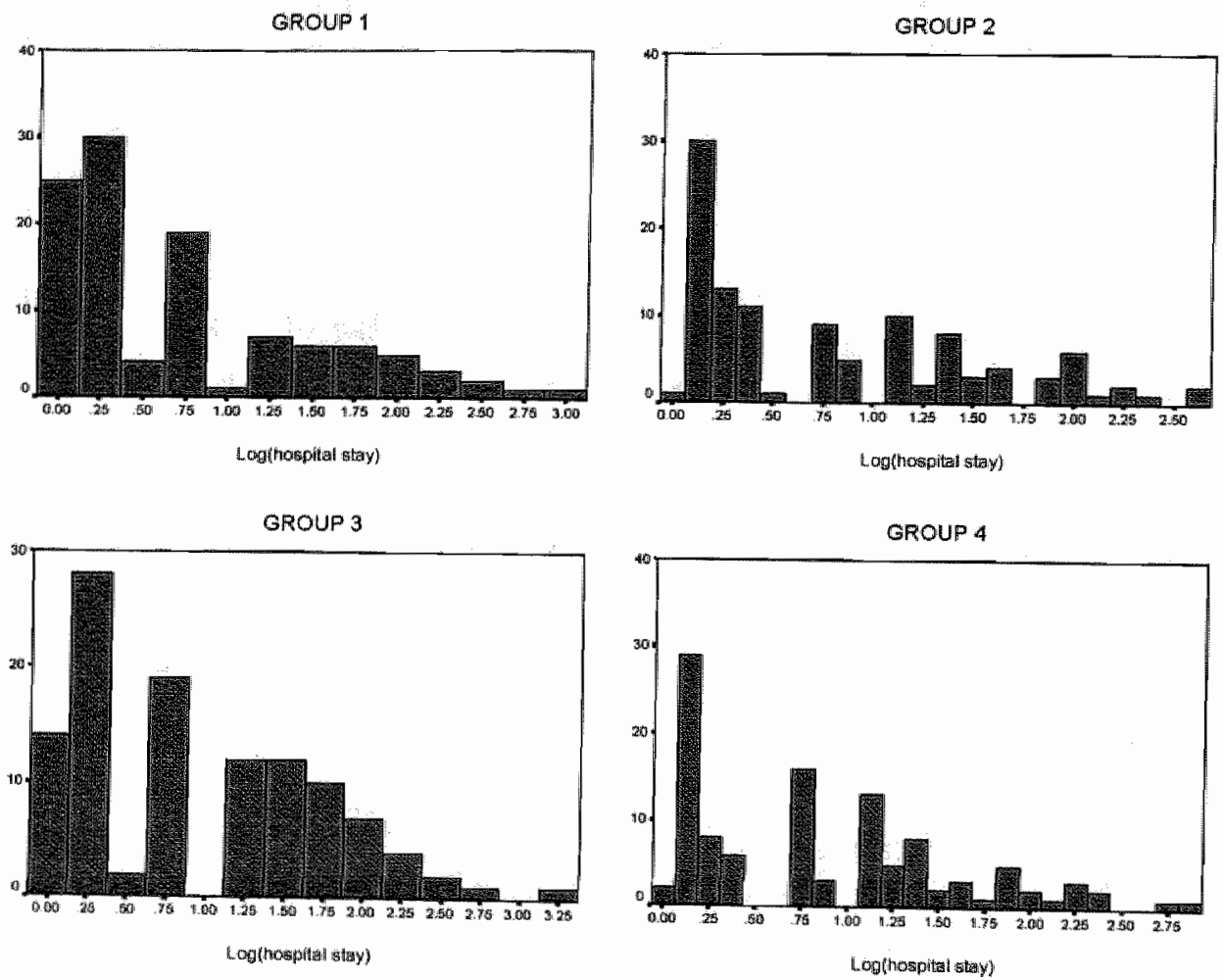

Figure 3.5 Histograms of duration of stay in hospital after log-transformation.

The ANOVA F-tests and two-sample $t$-tests on log-transformed and untransformed data as well as the Kruskal-Wallis and Mann-Whitney tests showed no significant differences between the mean durations of hospital stay. However, the corresponding $p$-values of the $F$ and $t$-tests on untransformed data sometimes differed much from those obtained with the other two methods (see Table 3.1).

This example shows that the log-transformation can indeed increase the confidence level of the $F$ - and $t$-tests when the data is positively skewed with a large coefficient of variation. But it also shows that the assumption of log-normality always has to be checked 
and non-parametric methods may be good alternatives to the parametric methods if this assumption is violated and no covariates are involved in the analysis.

Table 3.1 $F$ - and $t$-tests as well as non-parametric tests applied to untransformed and logtransformed durations of hospital stay.

\begin{tabular}{ccccc}
\hline \multirow{2}{*}{ Groups } & Df $(1)$ & \multicolumn{2}{c}{ Parametric } & Non-parametric \\
\cline { 2 - 5 } & Untransformed data & Log-transformed data & \\
\hline $1-2$ & $(3,441)$ & $F=1.07 ; \mathrm{p}=0.36$ & $F=1.81 ; \mathrm{p}=0.15$ & $K W=6.87 ; p=0.08$ \\
$1-3$ & 220 & $t=-0.15 ; \mathrm{p}=0.89(2)$ & $t=-0.83 ; \mathrm{p}=0.41$ & $M W=-1.45 ; p=0.15$ \\
$1-4$ & 219 & $t=-1.47 ; \mathrm{p}=0.14$ & $t=-2.15 ; \mathrm{p}=0.03$ & $M W=-2.40 ; p=0.02$ \\
$2-3$ & 222 & $t=-0.68 ; \mathrm{p}=0.50$ & $t=-1.54 ; \mathrm{p}=0.13$ & $M W=-2.03 ; p=0.04$ \\
$2-4$ & 221 & $t=-0.61 ; \mathrm{p}=0.54$ & $t=-0.73 ; \mathrm{p}=0.47$ & $M W=-0.63 ; p=0.53$ \\
$3-4$ & 221 & $t=0.88 ; \mathrm{p}=0.38$ & $t=0.69 ; \mathrm{p}=0.49$ & $M W=-0.52 ; p=0.60$ \\
\hline
\end{tabular}

() Notation: $\mathrm{Df}=$ degrees of freedom; $\mathrm{KW}=\mathrm{Kruskal}-\mathrm{Wallis}$; $\mathrm{MW}=\mathrm{Mann}-\mathrm{Whitney}$.

(2) All p-values in table are not corrected for multiple testing.

\subsection{Conclusions and discussion}

The present paper showed that the asymptotic relative efficiency (ARE) of the twosample $t$-test, ANOVA and ANCOVA on untransformed versus log-transformed data cam be considerably smaller than 1 when the data are log-normally distributed with homogeneous coefficients of variation across the groups. In case of two groups, the ARE of the two-sample t-test, ANOVA or ANCOVA on untransformed versus log-transformed data decreases with increasing standardized treatment effect $d=\left(\mu_{12}-\mu_{22}\right) / \sigma$, increasing correlation $\rho$ between the logarithms of the pre- $\left(y_{1}\right)$ and post-treatment measures $\left(y_{2}\right)$ and increasing coefficients of variation $\mathrm{CV}_{1}, \mathrm{CV}_{2}$ of the untransformed data. For example, if $\mathrm{CV}_{2} \geq 1$ and $d \geq 0.5$, then $\mathrm{ARE}$ of the two-sample $t$-test $\leq 0.65$. In addition, if $\mathrm{CV}_{1}=\mathrm{CV}_{2}$ and $\rho \geq 0.5$, then ARE of ANCOVA $\leq 0.60$. In case of three groups, the ARE of ANOVA decreases from 1.0 to roughly 0.7 when $\mathrm{CV}_{2}$ increases from 0.1 to 1.0 , depending on the effect sizes $d_{g g^{\prime}}$.

If the log-transformed data better satisfy the assumptions of the two-sample $t$-test, ANOVA or ANCOVA than the untransformed data, the log-transformation may increase the efficiency of the method. If the data are neither normally nor log-nomally distributed, then other statistical methods than those discussed in this paper, like nonparametric tests, bootstrap 
techniques, or other transformations, may have greater power. If the data are log-normally distributed, but the assumption of homogeneous coefficient of variation across groups is violated, then likelihood based methods, like the modified signed log-likelihood test, may be used to test the null hypothesis of equal arithmetic means (e.g. Wu et al., 2001). But the twosample t-test, ANOVA or ANCOVA without assuming homogeneous (residual) variances may still be applied to the log-transformed data to test the null hypothesis of equal geometric means or medians, which are less sensitive to skewness or outliers than the arithmetic means.

In practice, we frequently encountered log-normal data with a small coefficient of variation. As a consequence, a small gain in efficiency is obtained by applying the twosample $t$-tests, ANOVA or ANCOVA to the log-transformed instead of untransformed lognormal data. On the other hand, if the data are positively skewed and have a large coefficient of variation, the gain in efficiency can be large. But, as the example showed, non-parametric methods may then be a good alternative to the two-sample $t$-tests, ANOVA or ANCOVA applied to log-transformed data.

In conclusion, if the data are log-normally distributed with a homogeneous coefficient of variation across groups, a log-transformation can increase the asymptotic efficiency of the two-sample t-test, ANOVA or ANCOVA substantially. 


\section{References}

Barndorff-Nielsen, O. E. (1986). Inference on full and partial parameters, based on the standardized signed log likelihood ratio, Biometrika, 73, 307-322.

Barndorff-Nielsen, O. E. (1991). Modified signed log likelihood ratio, Biometrika, 78, 557563

Chelmow, D., Penzias, A.S., Kaufman, G., \& Cetrulo, C. (1995). Costs of triplet pregnancy, American Journal of Obsterrics and Genecology, 172, 677- 682.

Cohen, J. (1988). Statistical Power Analysis for the Behavioral Sciences (2nd ed.). Hillsdale, New Jersey: Lawrence Erlbaum.

Everitt, B. S. (1998). The Cambridge Dictionary of Statistics. Cambridge: Cambridge University Press.

Fleiss, J. L. (1986). The Design and Analysis of Clinical Trials. New York: John Wiley \& Sons.

Keene, O. N. (1995). The log transformation is special, Statistics in Medicine, 14, $811-819$.

Levy, K. J. (1978). An empirical comparison of the ANOVA $F$-test with alternatives which are more robust against heterogeneity of variance, Journal of Statistical Computing and Simulation, 8, 43-48.

Myles, P. S., Hunt, J. O., Fletcher, H., Smart, J. \& Jackson, T. (2000). Part I: Propofol, Thiopental, Sevofhrane, and Isoflurane - A randomized, controlled trial of effectiveness, Anesthesia and Analgesia, 91, 1163-1169.

Schouten, H. J. A. (1999). Sample size formula with a continuous outcome for unequal group sizes and unequal variances, Statistics in Medicine, 18,87-91.

Welch, B. L. (1951). On the comparison of several mean values: an al ternative approach, Biometrika, 38, 330-336.

Wilcox, R. R. (2001). Fundamentals of Modern Statistical Methods. New York: Springer, p 78-87.

Wu, J., Jiang, G., Wong, A. C. M. \& Sun, X. (2001). Likelihood analysis for the ratio of means of two independent log-normal distributions, Biometrics, 58, 463-469. 


\section{Chapter 3}

\section{Appendix}

\section{A1) Derivation of the correlation $R$ between untransformed pre-treatment measure $y_{1}$ and post-treatment measure $y_{2}$.}

By definition, $R^{2}=C_{g}^{2} / V_{1} V_{B 2,}$ where $C_{g}$ is the covariance between the pre- and posttreatment measures of group $g$ and $V_{l}, V_{g^{2}}>0$. This covariance is defined as $C_{8}=\mathrm{E}\left(y_{1} y_{2}\right)-\mathrm{E}\left(y_{1}\right) \mathrm{E}\left(y_{2}\right)$, where $\mathrm{E}\left(y_{1} y_{2}\right)=\mathrm{E}\left(\exp \left[\log \left(y_{1}\right)\right] \exp \left[\log \left(y_{2}\right)\right]\right]=\mathrm{E}[\exp (z)]$ and $z$ is defined as $z=\log \left(y_{1}\right)+\log \left(y_{2}\right)$, which is normally distributed. Then $\exp (z)$ is $\log$-nomally distributed with mean $\exp \left(\mu_{z}+\sigma_{z}{ }^{2} / 2\right)$, where $\mu_{z}=\mu_{1}+\mu_{g 2}$ and $\sigma_{z}^{2}=\sigma_{1}{ }^{2}+\sigma_{2}{ }^{2}+2 \rho \sigma_{1} \sigma_{2}$. Therefore,

$$
\begin{aligned}
& \quad C_{\mathrm{g}}=\exp \left(\mu_{1}+\mu_{g 2}+\sigma_{1}{ }^{2} / 2+\sigma_{2}{ }^{2} / 2+\rho \sigma_{1} \sigma_{2}\right)-\exp \left\{\mu_{1}+\sigma_{1}{ }^{2} / 2\right\} \exp \left\{\mu_{g 2}+\sigma_{2}{ }^{2} / 2\right\} \\
& =\exp \left\{\mu_{1}+\sigma_{1}{ }^{2} / 2\right\} \exp \left\{\mu_{g 2}+\sigma_{2}{ }^{2} / 2\right\}\left\{\exp \left(\rho \sigma_{1} \sigma_{2}\right)-1\right\}
\end{aligned}
$$

and

$$
R^{2}=\frac{\left\{\exp \left(\rho \sigma_{1} \sigma_{2}\right)-1\right\}^{2}}{\left\{\exp \left(\sigma_{1}^{2}\right)-1\right\}\left\{\exp \left(\sigma_{2}^{2}\right)-1\right\}}
$$

\section{A2) Sketched proof that $\left(1-\rho^{2}\right) /\left(1-R^{2}\right) \leq 1 .^{1}$}

Step $1\left(1-\rho^{2}\right) /\left(1-R^{2}\right) \leq 1$ if and only if $R^{2} / \rho^{2} \leq 1$.

Step 2. $R^{2} / \rho^{2}=f^{2}\left(\rho \sigma_{1} \sigma_{2}\right) /\left\{f\left(\sigma_{1}^{2}\right) f\left(\sigma_{2}^{2}\right)\right\} \leq f^{2}\left(\sigma_{1} \sigma_{2}\right) /\left(f\left(\sigma_{1}{ }^{2}\right) f\left(\sigma_{2}^{2}\right)\right\}$, where $f(\zeta) \equiv\{\exp (\zeta-1\}$ $1 \zeta$ is an increasing function of $\zeta(>0)$.

Step 3. $f^{2}\left(\sigma_{1} \sigma_{2}\right) /\left\{f\left(\sigma_{1}{ }^{2}\right) f\left(\sigma_{2}^{2}\right)\right\} \leq 1$ if and only if $g\left(\sigma_{1}, \sigma_{2}\right) \leq 0$, where $g\left(\zeta_{n} \varphi\right)=\{\exp (\zeta \phi)-1\}^{2}$ $-\left\{\exp \left(\zeta^{2}\right)-1\right\}\left\{\exp \left(\omega^{2}\right)-1\right\}(\zeta, \varphi>0)$.

Step 4. The partial derivatives of $g(\zeta, \varphi)$ are equal to 0 if and only if $\zeta=\varphi$.

Step 5. If $\zeta$ or $\varphi$ goes to infinity, $g(\zeta, \varphi)$ approaches - $\infty$. Thus, function $g(\zeta, \varphi)$ reaches a maximum for finite values of $\zeta$ and $\varphi$. Two possibilities: 1$)$ maximum is equal to $g(0, \varphi)=$ $g(6,0)=g(0,0)=0$ or 11$)$ maximum is reached where the partial derivatives are equal to 0 , which yields $\max \{g(\zeta, \varphi)\}=g(\zeta, \zeta)=0$. In both cases, $g(\zeta, \varphi) \leq 0$ for all $\zeta, \varphi>0$.

\footnotetext{
'A complete proof can be obtained from the first author by e-mail.
} 


\section{A3) Derivation of the pooled residual variance $V_{*}$}

The ANCOVA model that incorrectly assumes homogeneous slopes and residual variances can be written as

$$
y_{g i z}=\alpha_{g}+\beta\left(y_{g i 1}-M_{1}\right)+\varepsilon_{g i 2}
$$

where $\alpha_{g}$ and $\beta$ are the group-specific intercept and homogeneous slope of the regression line of group $g$, respectively. The errors $\varepsilon_{g i z}$ are assumed to be normally distributed with mean zero and homogeneous variance. The residual variance $V_{\varepsilon}$ is pooled across groups, i.e. $V_{s}=$ $\left\{\left(V_{12}-2 \beta C_{1}+\beta^{2} V_{1}\right)+\left(V_{22}-2 \beta C_{2}+\beta^{2} V_{1}\right)\right\} / 2$, where $\beta=\left(C_{1}+C_{2}\right) / 2 V_{1}$ and $C_{1}$ and $C_{2}$ are the covariances between untransformed pre-treatment measure $y_{1}$ and post-treatment measure $y_{2}$ within groups 1 and 2 , respectively. Thus,

$$
\begin{aligned}
V_{s} & =\frac{V_{12}+V_{22}}{2}-\frac{\left(C_{1}+C_{2}\right)^{2}}{4 V_{1}} \\
& =\frac{V_{12}+V_{22}}{2}\left(1-R^{2}\right)+\frac{R^{2}}{4}\left(\sqrt{V_{12}}-\sqrt{V_{22}}\right)^{2}
\end{aligned}
$$

which is larger than the mean of the heterogeneous residual variances, $\left(1-R^{2}\right)\left(K_{12}+V_{22}\right) / 2$.

A4) Derivation of the ARE of ANOVA applied to the untransformed versus logtransformed data in case of $G$ groups, i.e. equation (3.12).

The ANOVA F-test, assuming homogeneous within-group variances can be used to compare the means of the log-transformed data. Under the null hypothesis and assumption of equal group sizes, the F-statistic has a central, and under the alternative hypothesis the fistatistic has a non-central, $F$-distribution, both with $\nu_{1}=G-1$ and $\nu_{2}=G\left(n_{\mathrm{leg}}-1\right)$ degrees of freedom in the numerator and denominator, respectively. The non-centrality parameter $\delta$ is

$$
\delta=\sqrt{n_{\log } \sum_{\mathscr{B}}\left(\mu_{g 2}-\bar{\mu}_{2}\right)^{2}} / \sigma_{2}
$$




\section{Chapter 3}

In short, $\mathrm{H}_{6}: F \sim F_{w_{1}, v_{2}} ; \mathrm{H}_{\text {色 }}: F \sim F_{v_{1}, v_{2}}$ (e.g. Fleiss; 1986)

For the untransformed data, the Welch (1951) test, which assumes heterogeneous within-group variances, can be used. The Welch statistic is

$$
W=\frac{\sum V_{z 2}^{-1}\left(\bar{y}_{g 2}-\bar{y}_{w}\right)^{2} /(G-1)}{\left[1+\frac{2}{3}(G-2) \Lambda\right]},
$$

where

$$
\begin{aligned}
& \vec{y}_{g, 2}=\frac{1}{n_{\text {win }}} \sum_{g} y_{g i 2}, \bar{y}_{w}=\sum_{g} V_{g^{2}}^{-1} \bar{y}_{g, 2} / \sum_{g} V_{g^{2}}^{-1}, \\
& \Lambda=\frac{3 \sum_{g}\left(1-\frac{V_{g^{2}}^{-1}}{\sum_{g} V_{g^{2}}^{-1}}\right)^{2} /\left(n_{\mathrm{uta}}-1\right)}{G^{2}-1} .
\end{aligned}
$$

Under the null hypothesis, the Welch statistic $W$ has a central and under the alternative hypothesis, $W$ has a non-central $F$-distribution, both with $v_{1}=G-1$ and $v_{2}^{*}=1 / \Lambda$ degrees of freedom in the numerator and denominator, respectively (e.g. Levy, 1978). The non-centrality parameter $s^{*}$ is

$$
\delta^{*}=\sqrt{n_{\text {uี }} \sum_{s}\left(M_{g^{2}}-\bar{M}_{w}\right)^{2} / V_{g^{2}}},
$$

where

$$
\bar{M}_{w}=\left(\sum_{s} V_{g^{2}}^{m-1} M_{g^{2}}\right) /\left(\sum_{g} V_{g^{2}}^{-1}\right)
$$

Asymptotically, $v_{2}$ and $w_{2}^{*}$ go to infinity and the $F$-statistic and Welch statistic have the same central $\chi^{2}$-distribution divided by $v_{1}$ under the null hypothesis of equal means. Therefore, the critical value belonging to a pre-specified significance level $\alpha$ is the same for the $F$-test and Welch test for large $n$. As a consequence, assuming the same pre-specified 
power $1-\beta$, the $F$-statistic and Weich statistic must also have the same distribution under the alternative hypothesis. Additionally, under the altemative hypothesis, the $F$-statistic and Welch statistic have non-central $\chi^{2}$-distributions divided by $v_{1}$ with non-centrality parameters $\delta$ and $\delta^{*}$, respectively. Therefore, $F$ and $W$ have asymptotically the same distributions if and only if $\delta=\delta^{*}$. As a consequence,

$$
\mathrm{ARE}_{\mathrm{ANOVA}}=\frac{n_{\mathrm{log}}}{n_{\mathrm{un}}}=\frac{\sum_{8}\left(M_{g^{2}}-\bar{M}_{\mathrm{w}}\right)^{2} / V_{g^{2}}}{\sum\left(\mu_{g 2}-\bar{\mu}_{2}\right)^{2} / \sigma_{2}^{2}} .
$$


Chapter 3 
Optimal designs for estimation of linearly wivergent treatment efects

\section{Optimal designs in clinical trials with linearly divergent treatment effects}

This chapter has been accepted for publication in Contemporary Cinncal Trials with co-authors H. J. A. Schouten, G. J. P. van Breukelen and M. P. F. Berger 


\section{Abstract}

The effect of number of repeated measures on the variance of the generalized least squares (GLS) treatment effect estimator is considered assuming a linearly divergent treatment effect, equidistant time-points and either a fixed number of subjects or a fixed study budget: The optimal combination of group sizes and number of repeated measures is calculated by minimizing this variance subject to a linear cost function. For a fixed number of subjects, the variance of the GLS treatment effect estimator can be decreased by adding intermediate measures per subject. This decrease is relatively large if a) the covariance structure is compound symmetric or b) the structure approaches compound symmetry and the correlation between two repeated measures does not exceed 0.80 , or c) the correlation between two repeated measures does not exceed 0.60 if the time-lag goes to zero. In case the sample sizes and number of repeated measures are limited by budget constraints and the covariance structure includes a first-order auto-regression part, two repeated measures per subject yield highly efficient treatment effect estimators. Otherwise, it is more efficient to have more than two repeated measures. If the cowariance structure is unknown, the optimal design based on a first-order auto-regressive structure with measurement error is preferable in terms of robustness against misspecification of the covariance structure. The numerical results are illustrated by three examples.

Keywords: $\quad$ Cost Function; Generalized Least Squares; Group Sizes; Linear Divergence; Number of Repeated Measures. 


\subsection{Introduction}

In randomized clinical trials, the required number of subjects to obtain efficient treatment effect estimators will decrease when the number of repeated measures increases. The size of the decrease, however, depends on the covariance structure of the repeated measures. The question is now, if adding a subject costs much more than adding an intermediate measure, how many repeated measures and subjects within a particular budget yield the smallest variance of the treatment effect estimator?

Several papers have provided sample size formulae for repeated measures designs (e.g. Bloch, 1986; Hedeker, Gibbons \& Waternaux, 1999; Jung \& Ahn, 2003; Lui \& Cumberland, 1992; Overall \& Doyle, 1994; Overall \& Tonidandel, 2002; Yi \& Panzarella, 2002). Explicit formulae of the variance of the generalized least squares (GLS) treatment effect estimator have been derived assuming a compound symmetric (CS) or first-order autoregressive (AR1) covariance structure (e.g. Yi \& Panzarella, 2002). Costs for recruiting, treating and measuring subjects have been considered in a repeated measures analysis of variance model (e.g. Bloch, 1986; Lui \& Cumberland, 1992).

One purpose of the present paper is to discuss the variance of the GLS treatment effect estimator and derive variance formulae assuming a linearly increasing treatment effect and considering different covariance structures. Another purpose of the present paper is to calculate the combination of sample sizes and number of repeated measures that lead to the smallest variance of the treatment effect estimator for a certain covariance structure and given budget. Factors affecting such an optimal combination are the follow-up duration and timem points at which the repeated measures are taken. For simplicity, these follow-up duration and time-points will be assumed to be fixed and equidistant, respectively.

Our numerical results will be illustrated by three examples. In the first example, a randomized clinical trial was conducted to compare the effects of two additives to the rats' drinking water with no additive (control group) (Diggle, 1990). The main outcome was the body-weight of the rats. The second example is a randomized clinical trial in which the effect of docosahexaenoic acid (DHA) on retinal function is compared with placebo (Hoffman et al., 2004). The full-field cone electroretinograms (ERGs; the main outcome measure) were recorded for male patients with X-linked retinitis pigmentosa (XILRP). In the third example, an experiment was conducted to compare the effects of an existing antidepressant and placebo with a new antidepressant (Feighner, 1980). The main outcome was the score on the Hamilton 
Psychiatric Scale for Depression. In all three examples, a limearly divergent treatment effect was obtained and the main outcome variable was measured at five equally spaced time-points, where the first measure was taken at the start of the study. The question what the optimal design would have been for these studies will be addressed in the present paper.

In Section 4.2, the linear mixed effects model is introduced and some commonly used covariance structures for this model are discussed. In Section 4.3 , the variance of the GLS treatment effect estimator and the effect of adding intermediate measures on this variance; assuming a fixed number of subjects, is discussed for these covariance structures. In Section 4.4 , assuming a fixed total budget instead of a fixed number of subjects, the optimal number of repeated measures and sample sizes are calculated by minimizing the variance of the GLS treatment effect estimator subject to a linear cost function and different covariance structures. The numerical results are illustrated by an example in Section 4.5 . The conclusions are summarized and discussed in Section 4.6.

\subsection{Linear mixed effects model}

\subsubsection{Model}

For subject $i$, let $y_{i j}$ be the $j$ th repeated measurement of a continuous outcome variable measured at time $t_{j}(i=1,2, \ldots, N ; j=1,2, \ldots, K)$. The time-points $t_{j}$ are assumed to be the same for each subject. For simplicity, the first measure is taken at $t_{1}=0$, i.e. just before randomization, and the fixed total duration of the treatment period is rescaled to unity, i.e. $t_{K}=$ 1. Equidistant time-points are assumed, i.e. $t_{j}=(j-1) /(K-1)(j=1,2, \ldots, K)$. For subject $i$, a linear mixed effects model (e.g. Verbeke \& Molenberghs, 2000), can be written as

$$
y_{i}=X_{i} p+Z_{i} b_{i}+\varepsilon_{i}+e_{i}
$$

where $X_{i} p$ is the expected trend over time, $Z_{i} b_{i}$ is the subject-specific deviation from this expected trend, $\varepsilon_{i}$ is a component of auto-correlation and $e_{i}$ is an extra component of measurement error. The random effect vector $b_{i}$ and the error vectors $\varepsilon_{i}$ and $e_{i}$ are assumed to be independent and normally distributed with mean vectors $\theta$ and covariance matrices $D$, $\sigma_{s}^{2} H$ and $\sigma_{e}{ }^{2} I_{K}$, respectively, where $I_{K}$ is the $K$-dimensional identity matrix. If no component of auto-correlation is present, $\boldsymbol{H}=0$, i.e. a matrix containing only zeros. Otherwise a first- 
order auto-regressive correlation structure is considered for $\boldsymbol{H}$, i.e. the correlation $\rho_{\varepsilon}{ }^{\mathrm{t}}$ between $\varepsilon_{i j}$ and $\varepsilon_{i t^{\prime}}$ decreases with increasing time-lag $\tau=\left|t_{j}-t_{j}\right|=\left|j-j^{\prime}\right| /(K-1)$ and $\rho_{\varepsilon}$ is the correlation between $\varepsilon_{i 1}$ and $\varepsilon_{i K}$. The expected trend $X_{i} \beta$ is assumed to be homogeneous across groups, except for a linearly increasing treatment effect. The trend itself does not have to be linear, i.e.

$$
\boldsymbol{X}_{i} \boldsymbol{\beta}=\left(\begin{array}{ccccc}
1 & 0 & \cdots & 0 & G_{i} \times t_{1} \\
0 & \ddots & \ddots & \vdots & \vdots \\
\vdots & \ddots & \ddots & 0 & \vdots \\
0 & \cdots & 0 & 1 & G_{i} \times t_{K}
\end{array}\right)\left(\begin{array}{c}
\mu_{i} \\
\vdots \\
\mu_{k} \\
\beta
\end{array}\right),
$$

where $\beta$ is the linearly divergent treatment effect parameter and $G_{i}$ is an indicator of treatment, which is equal to 1 if subject $i$ belongs to the treatment group and equal to 0 if subject $i$ belongs to the control group. Thus, $y_{i j}$ has expectation $\mu_{j}$ in the control group and $\mu_{j}+\beta t_{j}$ in the treatment group. Due to randomization, no group effect is assumed at $t_{1}=0$. For a linear mixed effects model (4.1), the generalized least squares (GLS) estimator of $\beta$ is

$$
\hat{\boldsymbol{\beta}}^{\mathrm{GLS}}=\left(\sum_{i=1}^{N} \boldsymbol{X}_{i}^{\prime} \boldsymbol{V}_{i}^{-1} X_{i}\right)^{-1} \sum_{i=1}^{N} \boldsymbol{X}_{i}^{\prime} \boldsymbol{V}_{i}^{-1} \boldsymbol{y}_{i}
$$

where the covariance matrix $V_{j}$ of $y_{i}$ may consist of three parts: randorn effects $Z_{i} D Z_{i}^{\prime}$, autocorrelation $\sigma_{s}^{2} \boldsymbol{H}$ and measurement error $\sigma_{e}{ }^{2} I_{K}$ (e.g. Diggle, Liang \& Zeger, 1996, p. 79-80). For simplicity, the covariance matrix $V_{i}$ of $y_{i}$ is assumed to be homogeneous across persons and groups, i.e. $V_{i}=V$ for all $i$. The model-based variance of the GLS estimator of $\beta$ is

$$
\operatorname{Var}\left(\hat{\beta}^{\mathrm{oLs}}\right)=\left(\sum_{i=1}^{N} X_{i}{ }^{\prime} \boldsymbol{V}^{-\mathrm{i}} \boldsymbol{X}_{i}\right)^{-1}
$$

If each subject is measured twice, the variance of the GLS treatment effect estimator reduces to the same as the one obtained from analysis of covariance, i.e. $\left(N_{t}+N_{c}\right) /\left(N_{t} N_{c}\right)$ $\times V_{22}\left(1-V_{12}^{2} /\left(V_{11} V_{22}\right)\right\}$, where $N_{t}$ and $N_{c}$ are the number of subjects in treatment and control group, respectively, and $V_{k, l}$ is the $(k, l)$ element of $V(k, l=1,2)$ (e.g. Laird \& Wang, 1990). 


\section{Chapter 4}

\subsubsection{Covariance structures}

In practice, repeated measures data are often assumed to have a compound symmetric or first-order auto-regressive covariance structure. In some studies, however, other structures fit the data better. For example, in the rat-growth experiment (Diggle, 1990) a compound symmetric structure did not fit the data well and a first-order auto-regressive structure gave some estimation problems (see Section 4.5 ). Therefore, the following six covariance structures for the linear mixed effects model (4.1) based on random effects; a component of auto-correlation and/or measurement error (see also Table 4.1) will be considered:

1) $\mathrm{CS}$ or $\mathrm{RI}+\mathrm{ME}$ : a compound symmetric covariance structure can be obtained by a random intercept model with measurement error (see Table 4.1). In other words, the random part of $y_{i j}$ only consists of random intercept $b_{0 i}$ and measurement error $e_{i f}$ which have means zero and variances $\sigma_{0}{ }^{2}$ and $\sigma_{e}{ }^{2}$, respectively. As a result the variance $\left(\sigma_{0}{ }^{2}+\sigma_{e}{ }^{2}\right)$ of $y_{i j}$ and correlation $\rho_{j j^{\prime}}=\sigma_{0}{ }^{2} /\left(\sigma_{i j}{ }^{2}+\sigma_{e}{ }^{2}\right)$ between $y_{i j}$ and $y_{i j}$ are constant for all $j$ and $j^{\prime}(\neq j)$.

2) AR 1: first-order auto-regression $\varepsilon_{i j}$. The covariance matrix $V$ of $y_{i}$ only includes autocorrelation $\sigma_{\varepsilon}^{2} \boldsymbol{H}$, which implies a constant variance $\left(\sigma_{\varepsilon}{ }^{2}\right)$ and a decreasing correlation $\rho_{j j^{\prime}}=\rho_{i s}{ }^{\mathrm{r}}$ between $y_{i j}$ and $y_{i^{\prime}}$ with increasing time-lag $\tau=\left|t_{j}-t_{j^{\prime}}\right|$; the correllation between first and last measures $\rho_{1, K}=\rho_{\varepsilon}$ in this ARl case

3) AR 1+ME: first-order auto-regression $\varepsilon_{i j}$ and measurement error $e_{i j}$. The covariance matrix $\boldsymbol{V}$ of $y_{t}$ consists of auto-correlation $\sigma_{5}^{2} \boldsymbol{H}$ and measurement error $\sigma_{a}^{2} I_{K}$, which implies a constant variance $\left(\sigma_{\varepsilon}{ }^{2}+\sigma_{e}{ }^{2}\right)$ of $y_{l i}$ and a decreasing correlation $\rho_{j j^{n}}=$ $\rho_{\varepsilon}{ }^{r} \sigma_{E}{ }^{2} /\left(\sigma_{\varepsilon}{ }^{2}+\sigma_{\varepsilon}{ }^{2}\right)$ with increasing time-lag $\tau$. Due to measurement error, the correlation between two repeated measures is bound to a maximum $<1$, i. e the correlation does not approach 1 if the time-lag goes to zero.

4) RI+ARI: random intercept $b_{0 i}$ and first-order auto-correlation $\varepsilon_{i j}$. The random part of $y_{i j}$ is $\left(b_{0 i}+\delta_{i j}\right)$, which implies a constant variance $\left(\sigma_{0}{ }^{2}+\sigma_{6}^{2}\right)$ of $y_{i j}$ and a decreasing correlation $\rho_{j j^{\prime}}=\left(\sigma_{i 0^{2}}{ }^{2}+\rho_{\varepsilon}{ }^{\tau} \sigma_{\varepsilon}{ }^{2}\right) /\left(\sigma_{0}{ }^{2}+\sigma_{\varepsilon}{ }^{2}\right)$ with increasing time-lag $\tau$. Due to the random intercept, the correlation $\rho_{j,}$ between two repeated measures is bound to a minimum $>$ 0 , i.e. $p_{i l^{2}}$ approaches $\sigma_{0}{ }^{2} /\left(\sigma_{n}{ }^{2}+\sigma_{\varepsilon}{ }^{2}\right)>0$, if the time-lag $\tau$ goes to infinity.

5) RI+ARI+ME: random intercept $b_{0 i}$, first-order auto-correlation $\varepsilon_{i j}$ and measurement error $e_{i j}$. The random part of $y_{i j}$ is now $\left(b_{0 i}+\varepsilon_{i j}+e_{i j}\right)$, which implies a constant variance $\left(\sigma_{0}{ }^{2}+\sigma_{\varepsilon}{ }^{2}+\sigma_{e^{2}}{ }^{2}\right)$ and decreasing correlation $\rho_{b,^{\prime \prime}}=\left(\sigma_{0}{ }^{2}+\rho_{\varepsilon}{ }^{5} \sigma_{\varepsilon}{ }^{2}\right) /\left(\sigma_{0}{ }^{2}+\sigma_{\varepsilon}{ }^{2}+\sigma_{e}{ }^{2}\right)$ 
with increasing time-lag $\tau$. Due to the random intercept and measurement error, this correlation $\rho_{j j^{\prime}}$ is bound to a minimum $>0(\tau=\infty)$ and a maximum $<1(\tau=0)$.

6) $\mathrm{RI}+\mathrm{RS}+\mathrm{ME}$ : random intercept $b_{0 i}$ and random slope $b_{v i}$ plus measurement error $e_{j y}$. The random part of $y_{i j}$ is $\left(b_{0 i}+b_{1 i} t_{j}+e_{i j}\right)$, where $\left(b_{0 i}+b_{1 i} t_{j}\right)$ is a linear deviation from the expected trend $\boldsymbol{X} \beta$ (see equation (4.2)). The random intercept and slope, $b_{0 \text { ind }}$ $b_{1}$, are normally distributed with mean zero, variances $\sigma_{0}{ }^{2}$ and $\sigma_{1}{ }^{2}$ and covariance $\sigma_{01}$. If the time-lag $\tau$ between $y_{i j}$ and $y_{i j^{\prime}}$ increases, the correlation $\rho_{j^{\prime}}$ (see Appendix A2) can decrease or increase or both, depending on the covariance parameters $\sigma_{0}{ }^{2}, \sigma_{1}{ }^{2}, \sigma_{01}$ and $\sigma_{\varepsilon}{ }^{2}$. Further, the covariance structure is non-stationary, i.e the variance of $y_{i j}$ varies over time and the correlation $\rho_{j, f^{*}}$ between two repeated measures not only depends on time-lag (e.g. $\left.\rho_{1,2} \neq \rho_{1,3}\right)$ but also on time (e.g. $\left.\rho_{1,2} \neq \rho_{2,3}\right)$.

Table 4.1 Covariance structures for model (4.1), based on random effects, auto-correlation and/or measurement error.

\begin{tabular}{cccc}
\hline Covariance structure & $D$ & $H$ & Measurement error $\sigma_{a}{ }^{2} \boldsymbol{I}_{K}$ \\
\hline $\mathrm{CS}=\mathrm{RI}+\mathrm{ME}$ & $\left(\sigma_{0}{ }^{2}\right)$ & 0 & Yes \\
AR1 & 0 & AR1 & No \\
AR1+ME & 0 & AR1 & Yes \\
RI+AR1 & $\left(\sigma_{0}{ }^{2}\right)$ & ARl & No \\
RI+AR1+ME & $\left(\sigma_{0}{ }^{2}\right)$ & AR1 & Yes \\
RI+RS+ME & $\left(\sigma_{0}^{2} \sigma_{01}\right.$ & 0 & Yes \\
\hline
\end{tabular}

Notation: $\theta$ is a matrix containing only zeros; $\boldsymbol{H}=\mathrm{AR} 1$ means that $\boldsymbol{\varepsilon}_{i}$ has a first-order auto regressive covariance structure with variance $\sigma_{E}{ }^{2}$ and a correlation $\rho_{g}{ }^{5}$ between $\varepsilon_{i y}$ and $F_{i j}$, where the time-lag $\tau=\left|t_{j}-t_{j}\right|=\left|j-f^{t}\right| /(K-1)$ and $\rho_{\varepsilon}$ is the correlation between $\varepsilon_{i 1}$ and $\varepsilon_{i}$.

The differences between the five stationary covariance structures are illustrated in Figure 4.1 , where the correlation $\rho_{y j^{\prime}}$ between $y_{i j}$ and $y_{y^{\prime}}$ is plotted as function of the time-lag $\tau$ $=\left|t_{j}-t_{j}\right|$. The covariance parameter values were chosen such that realistic correlation structures were obtained, i.e. $\sigma_{0}{ }^{2}=0.60$ and $\sigma_{\mathcal{s}}{ }^{2}=0.40$ for $\mathrm{CS}, \sigma_{\mathbb{E}}{ }^{2}=1$ and $\rho_{\varepsilon}=0.20$ for AR1, $\sigma_{\varepsilon}^{2}=0.8, \sigma_{e}^{2}=0.2$ and $\rho_{\varepsilon}=0.50$ for $A R 1+M E, \sigma_{0}{ }^{2}=0.15, \sigma_{\varepsilon}^{2}=0.85$ and $\rho_{\varepsilon}=0.06$ for RI+AR1, $\sigma_{0}{ }^{2}=0.30, \sigma_{\varepsilon}{ }^{2}=0.50, \sigma_{i}{ }^{2}=0.20$ and $\rho_{\varepsilon}=0.20$ for RI+ARI+ME. Notice that, without loss of generality, $\operatorname{Var}\left(y_{i j}\right)=1$ for all five stationary covariance structures. Since $\rho_{\varepsilon}=$ 
0.20 for AR1 and $\rho_{z}=0.06$ for $\mathrm{RI}+\mathrm{AR} 1$, the correlation $\rho_{j y}$ is smaller for $\mathrm{R}+\mathrm{AR} 1$ than for $A R \rrbracket$ when $0<\tau<1$. The opposite would be true if the error correlation $\rho_{\varepsilon}$ was the same in borh cases.

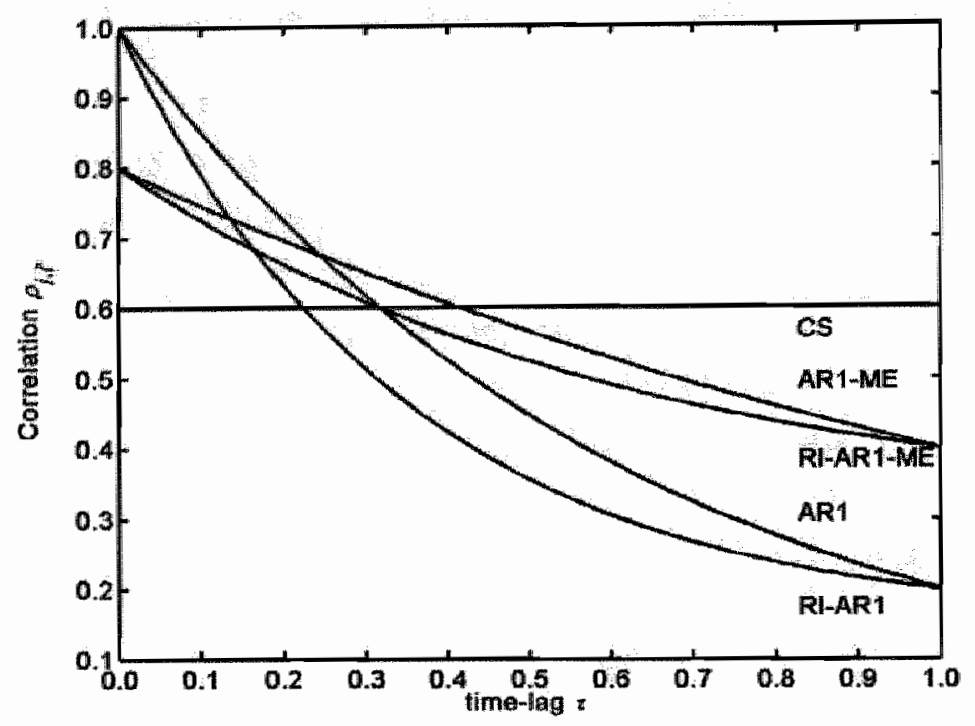

Figure 4.1 Correlation $\rho_{j, j}$ between $y_{i j}$ and $y_{i, j}$, where the time-lag $\tau=\left|t_{j}-t_{j}\right|$

Figure 4.1 shows the effects of the three parts of the covariance structure: random effects, auto-correlation and measurement error. Auto-correlation yields a decreasing correlation $\rho_{i y^{\prime}}$ with increasing time-lag $\tau$ and measurement error yields an upper bound $<1$ for the correlation, i.e. the correlation approaches a maximum $<1$ if the time-lag goes to zero. Due to the random intercept, the correlation $\rho_{j j^{\prime}}$ approaches a minimum $>0$ if the time-lag goes to infinity. The effect of the random intercept on the correlation $\rho_{j, j^{\prime}}$ however, is relatively snall compared to the effect of measurement error (see Figure 4.1 ), because the effect of measurement error is maximal for time-lag $\tau=0$, whereas the effect of a random intercept is minimal for $\tau=0$ and maximal for an infinite time-lag. 


\subsection{Variance of GLS treatment effect estimator}

\subsection{Variance formulae}

Variance formulae of the GLS treatment effect estimator (equation (4.4)) are derived for $\mathrm{RI}+\mathrm{AR} 1$ and RI+RS+ME covariance structures (see Table 4.2 for formulae and Appendix A1 for derivations). Variance formulae for CS and AR1 covariance structures can be obtained from the formula for $\mathrm{RI}+\mathrm{AR} 1$, because $\mathrm{RI}+\mathrm{AR} 1$ reduces to $\mathrm{CS}$ if $\rho_{\varepsilon}=0$ and to AR1 if $\sigma_{0}{ }^{2}=0$. Further, RI+RS+ME reduces to $\mathrm{CS}$, if $\sigma_{01}=\sigma_{1}^{2}=0$.

No variance formulae for $\mathrm{AR} 1+\mathrm{ME}$ and $\mathrm{RI}+\mathrm{AR} 1+\mathrm{ME}$ covariance structures could be derived (see Appendix A1). Therefore, the variance of the GLS treatment effect estimator was calculated numerically with equation (4.4) for each combination of number of repeated measures $K$ and correlation $\rho_{1, K}$ between first and last measures in the next sections.

Table 4.2 Variance of GLS treatment effect estimator under RI+AR1 and RI+RS+ME covariance structures $\left(N_{t}\right.$ and $N_{c}$ are the number of subjects in treatment and control group, respectively).

\begin{tabular}{|c|c|}
\hline $\begin{array}{c}\text { Covariance } \\
\text { structure }\end{array}$ & $\operatorname{Var}\left(\hat{\beta}^{\mathrm{GLS}}\right)$ \\
\hline $\mathrm{RI}+\mathrm{AR} 1$ & $\frac{N_{f}+N_{c}}{N_{p} N_{c}}\left(1-\rho_{s}^{2 a(K-1)}\right) \sigma_{s}^{2} \times \frac{12(K-1)\left\{\left(1+\rho_{E}^{1 /(K-1)}\right) \sigma_{s}^{2}+\left[K-(K-2) \rho_{s}^{1 /(K-1)}\right] \sigma_{0}^{2}\right\}}{A \sigma_{s}^{2}+B \sigma_{0}^{2}}$ \\
\hline & $\begin{aligned} A= & 2\left(1+\rho_{s}^{1 /(K-1)}\right)\left[(K-2)(2 K-3) \rho_{s}^{2(K N)}-4 K(K-2) \rho_{s}^{1 /(K-1)}+K(2 K-1)\right] \\
B= & -(K-2)^{2}(K-3) \rho_{s}^{3 /(K-1)}+(K-2)(K-3)(3 K+2) \rho_{s}^{2(K-1)} \\
& -K(K+1)(3 K-8) \rho_{s}^{1 /(K-1)}+K^{2}(K+1)\end{aligned}$ \\
\hline $\mathrm{RI}+\mathrm{RS}+\mathrm{ME}$ & $\frac{N_{t}+N_{c}}{N_{i} N_{c}} \times \frac{K^{2}(K+1)\left(\sigma_{0}^{2} \sigma_{1}^{2}-\sigma_{01}^{2}\right)+2 \sigma_{0}^{2} E}{K^{2}(K+1) \sigma_{0}^{2}+2 K(2 K-1) \sigma^{2}}$ \\
\hline & $E=6 K(K-1)\left(\sigma_{0}^{2}+\sigma_{01}\right)+K(2 K-1) \sigma_{1}^{2}+6(K-1) \sigma_{0}^{2}$ \\
\hline
\end{tabular}


(a)

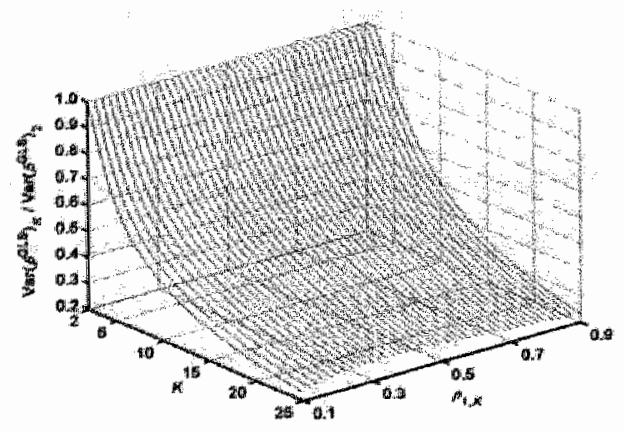

(c)

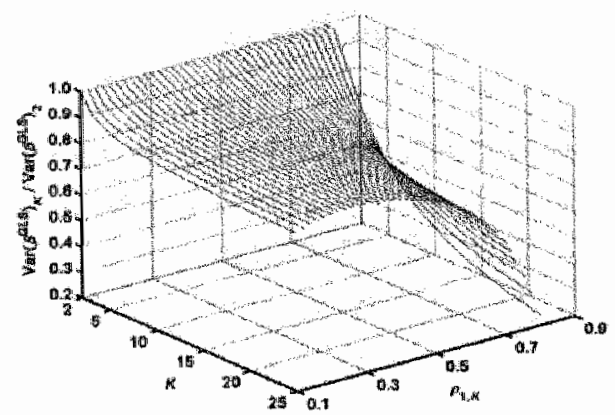

(b)

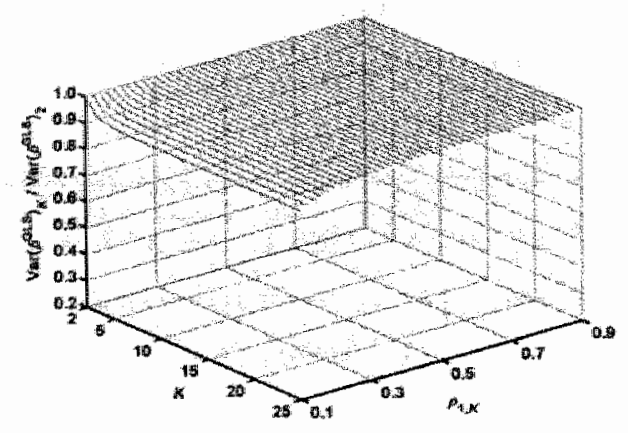

(d)

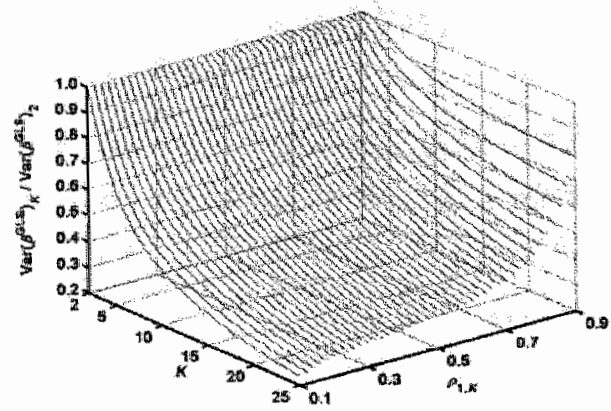

Figure 4.2 Relative efficiency of GLS treatment effect estimators based on 2 versus $K$ repeated measures under different covariance structures: a) $C S$, b) $A R 1$, c) $A R 1+M E$ with $\sigma_{\varepsilon}{ }^{2}$ $=0.80$ and $\left.\sigma_{e}{ }^{2}=0.20, \mathrm{~d}\right) \mathrm{R}+\mathrm{RS}+\mathrm{ME}$, with $\sigma_{00}{ }^{2}=0.7, \sigma_{1}{ }^{2}=0.1, \sigma_{01}=-0.1$ and $\sigma_{e}{ }^{2} \in[.01854$, 5.35021 .

\subsubsection{Reswils}

In the present paper, the relative efficiency of the GLS treatment effect estimators based on two versus $K$ repeated measures is defined as the inverse ratio of the variances of these estimators. This relative efficiency is plotted as function of $K$ and correlation $\rho_{1, K}$ between first and last measures for CS, AR1, ARI+ME and RI+RS covariance structures in Figures $4.2 \mathrm{a}-4.2 \mathrm{~d}$, respectively. The figures for RI+AR1 and RI+AR1+ME structures were omitted, because they were similar to those for the ARI and AR1+ME structures, respectively, as was expected from Figure 4.1. In Figure 4.2 , the sample sizes $N_{t}$ and $N_{c}$ are 
assumed fixed. Notice that the relative efficiency of two versus $K$ repeated measures, tie the ratio of variances of the GLS treatment effect estimators, is then not influenced by the actual sample sizes, because both variances are proportional to $\left(N_{t}+N_{c}\right) /\left(N_{t} N_{c}\right)$ (see Table 4.2 and Appendix Al). Variance and correlation values are considered that are not too extrente to maintain the differences between the specific covariance structures. For example, the relative efficiency is plotted for the AR $1+M E$ structure with $\sigma_{\varepsilon}{ }^{2}=0.80, \sigma_{e}{ }^{2}=0.20$ (the maximal correlation is 0.80 ). Similar conclusions were obtained for $0.40 \leq \sigma_{\varepsilon}{ }^{2} \leq 0.90\left(0.60 \geq \sigma_{e}{ }^{3} \geq\right.$ 0.10). If $\sigma_{\varepsilon}{ }^{2}<0.40$ or $\sigma_{\varepsilon}{ }^{2}>0.90$, however, the AR1+ME structure resembles a CS or an ARI structure, respectively.

Although the relative efficiency of the GLS treatment effect estimators based on two versus $K$ repeated measures decreases with increasing $K$, the amount of decrease depends strongly on the covariance structure. The decrease is relatively large for a compound symmetric (CS) structure (Figure 4.2a), whereas it is small for a first-order auto-regressive (AR1) structure (Figure 4.2b). For a first-order auto-regressive structure with measurement error (ARI+ME), the benefit of adding intermediate measures increases 1) with increasing auto-correlation $\rho_{s}$ (see Figure $4.2 \mathrm{c}$ and note that $\rho_{\mathrm{l}, K}$ increases with increasing $\rho_{s}$ ) and/or 2) with increasing measurement error $\sigma_{e}{ }^{2}$ (not shown here). For example, for the ARI+ME structure with $\sigma_{\varepsilon}{ }^{2}=0.80, \sigma_{\varepsilon}{ }^{2}=0.20$, the relative efficiency of 2 versus 25 repeated measures exceeds 0.80 if $\rho_{L, K} \leq 0.50$ and the relative efficiency is below 0.60 if $\rho_{1, K} \geq 0.70$. If $\sigma_{e}{ }^{2} \geq 0.40$ instead of $\sigma_{e}{ }^{2}=0.20$ (the maximal correlation $\leq 0.60$ instead of $\leq 0.80$ ), however, the relative efficiency of 2 versus 25 repeated measures is less than 0.70 for all $\rho_{1, K} \in[0,10,0.90]$. This can be explained as follows. If $\rho_{\varepsilon}$ and/or $\sigma_{e}{ }^{2}$ increases, the difference between the maxima! correlation (obtained when the time-lag $\tau=0$ ) and the correlation $\rho_{1, K}$ between first and last measures decreases (see Section 4.2.2) and the AR.1+ME structure approaches the CS structure. Finally, Figure $4.2 \mathrm{~d}$ shows that for a RI+RS+ME structure with $\rho_{1, K}<0.90$, the relative efficiency of two versus $K$ repeated measures decreases considerably with increasing $K$. For example, if $\rho_{1, K} \leq 0.88$, the relative efficiency of 2 versus 25 repeated measures is less than or equal to 0.70 . Further, if the measurement error variance $\sigma_{e}{ }^{2}$ increases, i.e. $\rho_{1, K}$ decreases, the benefit of adding intermediate measures increases, because then $\mathrm{RI}+\mathrm{RS}+\mathrm{ME}$ approaches CS (see Appendix A2).

In conclusion, although the variance of the GLS treatment effect estimator decreases if intermediate measures per subject are added, this decrease is relatively large only if a) the covariance structure is compound symmetric or b) the structure approaches compound 
symmetry and the correlation between two repeated measures does not exceed 0.80 , or c) the correlation between two repeated measures does not exceed 0.60 , if the time-lag goes to zero.

The logic behind this conclusion is as follows. If intermediate measures are added, the time-lag $\tau=\left|t_{j}-t_{j+1}\right|=1 /(K-1)$ between successive measures decreases and the correlation between two successive measures increases. If the correlation between successive measures is close to 1 , adding intermediate measures hardly decrease the variance of the GLS treatment effect estimator. If the correlation between successive measures is bound to a maximum of 0.80 or smaller, however, adding intermediate measures can decrease the variance of the GLS treatment effect estimator, even if the time-lag between successive measures becomes smaller.

\subsection{Optimal number of repeated measures and sample sizes}

\subsubsection{Cost function}

In practice, the number of subjects and repeated measures per subject are often limited by budget constraints. Therefore, the optimal combination of sample sizes $N_{t}, N_{c}$ and number of repeated measures per subject $K$ will be calculated by minimizing the variance of the GLS treatment effect estimator subject to a linear cost function,

$$
C=C_{0}+C_{1} N_{t}+C_{2} N_{c}+C_{3} N K_{s}
$$

where $N=N_{t}+N_{c}$ is the total number of subjects, $C$ is the total budget for sampling and measuring subjects, $C_{0}$ is the overhead cost, $C_{1}$ and $C_{2}$ are the costs per subject in treatment and control group, respectively, i e cost for recruitment and cost of treatment, and $C_{3}$ is the cost per repeated measure. Given this cost function, minimizing the variance of the treatment effect estimator within a certain maximal allowable budget is equivalent to minimizing the budget given a maximal allowable variance of the treatment effect estimator (e.g. Moerbeek, van Breukelen \& Berger, 2000).

The costs per subject may be higher in the treatment group $C_{1}$ than in the contral group $C_{2}$. In that case, randomizing a smaller fraction $f=N_{t} /\left(N_{t}+N_{c}\right)$ of subjects to the treatment group and a higher fraction $(1-f)=N_{c} /\left(N_{t}+N_{c}\right)$ to the control group results in lower costs of the study to obtain the same variance of the treatment effect estimator. If the subject costs $C_{1}$ and $C_{2}$ are relatively large compared to the cost $C_{3}$ per repeated measure, more repeated measures and fewer subjects may also result in lower study costs without an increase 
in variance. Therefore, a relatively large range of cost ratios $C_{1} / C_{3}(=1,10,100)$ and $C_{3} / C_{3} C$ $1,10,100)$ are considered.

The optimization problem reduces to minimizing the variance of the GLS treatment effect estimator (equation (4.4) or Table 4.2) as function of $f$ and $K$. To see this, first rewrite equation (4.5) as $N=\left(C-C_{0}\right) /\left\{C_{1} f+C_{3}(1-f)+C_{3} K\right\}$. Next, observe that the variance of the GLS treatment effect estimator is proportional to $\left(N_{t}+N_{c}\right) /\left(N_{i} N_{c}\right)=1 /\{f 1-f) N$ (e.g. see Table 4.2), which can be rewritten as $\left\{C_{f} f+C_{2}(1-f)+C_{3} K\right\} /\left\{(1-f)\left(C-C_{0}\right)\right\}$ by plugging in the first result. If $C_{1} \neq C_{2}$ and $K$ is fixed, the optimal fraction $f_{\text {opt }}$ that minimizes the variance is

$$
\left.f_{\mathrm{opt}}=\left\{\sqrt{\left(K+C_{1} / C_{3}\right)\left(K+C_{2} / C_{3}\right)}-\left(K+C_{2} / C_{3}\right)\right\} /\left(C_{1} / C_{3}\right)-\left(C_{2} / C_{3}\right)\right\}
$$

If $C_{1}=C_{2}$, the optimal fraction $f_{\text {opt }}=1 / 2$ for all $K$. Using this optimal fraction fopt and given covariance parameters, the optimal number of repeated measures $K_{\text {opt }}$ is calculated by minimizing the variance of the GLS treatment effect estimator for different cost ratios $C_{1} / C_{2}$ and $C_{2} / C_{3}$ (see Tables $43,4.4$ and 4,5). For practical reasons, $K$ is bounded to a maximum of 25 repeated measures.

Since the results were hardly affected by adding a random intercept to an ARI or ARI+ME structure, the results for RI+AR1 and RI+AR1+ME structures were omitted. The chosen covariance parameters in Tables $4.3,4.4$ and 4.5 ensure that the correlation between first and last measures $\rho_{1, K}$ may vary between 0.1 and 0.9 . 
Table 4.3 Optimal number of repeated measures $K_{\text {opt }} \leq 25$ (relative efficiency of 2 versus $K_{\text {opt }}$ repeated measures) for $C_{1} / C_{2}=1, f=1 / 2(1)$.

\begin{tabular}{|c|c|c|c|c|c|c|}
\hline \multirow{2}{*}{$\begin{array}{c}\text { Covariance } \\
\text { structure }\end{array}$} & \multirow[b]{2}{*}{$C_{2} / C_{3}$} & \multicolumn{5}{|c|}{ Correlation between first and last measures $\rho_{1, K}$} \\
\hline & & 0.1 & 0.3 & 0.5 & 0.7 & 0.9 \\
\hline $\mathrm{CS}$ & 1 & $2(1.00)$ & $2(1.00)$ & $2(1.00)$ & $2(1.00)$ & $2(1.00)$ \\
\hline & 10 & $17(0.67)$ & $25(0.80)$ & $25(0.78)$ & $25(0.73)$ & $25(0.67)$ \\
\hline \multirow{4}{*}{ ARI } & 100 & $25(0.28)$ & $25(0.34)$ & $25(0.33)$ & $25(0.31)$ & $25(0.28)$ \\
\hline & 1 & $2(1.00)$ & $2(1.00)$ & $2(1.00)$ & $2(1.00)$ & $2(1.00)$ \\
\hline & 10 & $2(1.00)$ & $2(1.00)$ & $2(1.00)$ & $2(1.00)$ & $2(1.00)$ \\
\hline & 100 & $4(0.95)$ & $3(1.00)$ & $2(1.00)$ & $2(1.00)$ & $2(1.00)$ \\
\hline \multirow[t]{2}{*}{$\mathrm{AR} 1+\mathrm{ME}^{(2)}$} & 1 & $2(1.00)$ & $2(1.00)$ & $2(1.00)$ & $2(1.00)$ & $2(1.00)$ \\
\hline & 10 & $2(1.00)$ & $2(1.00)$ & $2(1.00)$ & $2(1.00)$ & $15(0.92)$ \\
\hline 3 & 100 & $5(0.94)$ & $3(1.00)$ & $2(1.00)$ & $9(0.98)$ & $25(0.40)$ \\
\hline \multirow[t]{3}{*}{$\mathrm{RI}+\mathrm{RS}+\mathrm{ME}$} & 1 & $2(1.00)$ & $2(1.00)$ & $2(1.00)$ & $2(1.00)$ & $2(1.00)$ \\
\hline & 10 & $16(0.67)$ & $18(0.85)$ & $1.5(0.92)$ & $2(1.00)$ & $2(1.00)$ \\
\hline & 100 & $25(0.29)$ & $25(0.36)$ & $25(0.40)$ & $25(0.49)$ & $12(0.97)$ \\
\hline
\end{tabular}

Table 4.4 Optimal number of repeated measures $K_{\text {op }} \leq 25$ (relative efficiency of 2 versus $K_{\text {opt }}$ repeated measures) for $C_{1} / C_{2}=10$ and $f_{\text {opt }}$ is function of $K$ (equation (4.6))

\begin{tabular}{ccccccr}
\hline $\begin{array}{c}\text { Covariance } \\
\text { structure }\end{array}$ & $C_{2} / C_{3}$ & \multicolumn{6}{c}{0.1} & \multicolumn{5}{c}{ Correlation between first and last measures $p_{1, k}$} \\
\hline $\mathrm{CS}$ & 1 & $8(0.92)$ & $2(1.00)$ & $2(1.00)$ & $2(1.00)$ & \multicolumn{1}{c}{0.9} \\
& 10 & $25(0.37)$ & $25(0.44)$ & $25(0.42)$ & $25(0.40)$ & $25(0.37)$ \\
& 100 & $25(0.25)$ & $25(0.30)$ & $25(0.29)$ & $25(0.27)$ & $25(0.25)$ \\
AR1 & 1 & $2(1.00)$ & $2(1.00)$ & $2(1.00)$ & $2(1.00)$ & $2(1.00)$ \\
& 10 & $3(0.98)$ & $2(1.00)$ & $2(1.00)$ & $2(1.00)$ & $2(1.00)$ \\
& 100 & $6(0.93)$ & $3(0.99)$ & $2(1.00)$ & $2(1.00)$ & $2(1.00)$ \\
AR1+ME & 1 & $2(1.00)$ & $2(1.00)$ & $2(1.00)$ & $2(1.00)$ & $2(1.00)$ \\
& 10 & $3(0.97)$ & $2(1.00)$ & $2(1.00)$ & $2(1.00)$ & $25(0.52)$ \\
& 100 & $8(0.91)$ & $8(0.98)$ & $12(0.98)$ & $25(0.91)$ & $25(0.35)$ \\
RII+RS+ME & 1 & $8(0.92)$ & $2(1.00)$ & $2(1.00)$ & $2(1.00)$ & $2(1.00)$ \\
& 10 & $25(0.37)$ & $25(0.47)$ & $25(0.52)$ & $25(0.63)$ & $2(1.00)$ \\
& 100 & $25(0.25)$ & $25(0.31)$ & $25(0.35)$ & $25(0.42)$ & $25(0.85)$ \\
\hline
\end{tabular}


Table 4.5 Optimal number of repeated measures $K_{\text {opt }}$ (relative efficiency of 2 versus $K_{\text {opt }}$ repeated measures) for $C_{1} / C_{2}=100$ and $f_{\text {opt }}$ is function of $K$ (equation (4.6)) ${ }^{(1)}$.

\begin{tabular}{|c|c|c|c|c|c|c|}
\hline \multirow{2}{*}{$\begin{array}{c}\text { Covariance } \\
\text { Structure }\end{array}$} & & \multicolumn{5}{|c|}{ Correlation between first and last measures $\rho_{1, K}$} \\
\hline & $C_{2} / C_{3}$ & 0.1 & 0.3 & 0.5 & 0.7 & 0.9 \\
\hline \multirow[t]{3}{*}{$\mathrm{CS}$} & 1 & $25(0.48)$ & $25(0.56)$ & $25(0.55)$ & $25(0.51)$ & $25(0.47)$ \\
\hline & 10 & $25(0.27)$ & $25(0.32)$ & $25(0.31)$ & $25(0.29)$ & $25(0.27)$ \\
\hline & 100 & $25(0.24)$ & $25(0.28)$ & $25(0.27)$ & $25(0.25)$ & $25(0.24)$ \\
\hline \multirow[t]{3}{*}{ AR 1} & 1 & $2(1.00)$ & $2(1.00)$ & $2(1.00)$ & $2(1.00)$ & $2(1.00)$ \\
\hline & 10 & $4(0.95)$ & $3(1.00)$ & $2(1.00)$ & $2(1.00)$ & $2(1,00)$ \\
\hline & 100 & $8(0.93)$ & $5(0.99)$ & $3(1.00)$ & $2(1.00)$ & $2(1.00)$ \\
\hline \multirow[t]{3}{*}{$\mathrm{ARI}+\mathrm{ME}^{(2)}$} & 1 & 3. $(0.99)$ & $2(1.00)$ & $2(1.00)$ & $2(1.00)$ & $25(0.62)$ \\
\hline & 10 & $5(0.93)$ & $4(0.99)$ & $2(1.00)$ & $12(0.97)$ & $25(0.38)$ \\
\hline & 100 & $16(0.89)$ & $21(0.96)$ & $25(0.94)$ & $25(0.87)$ & $25(0.33)$ \\
\hline \multirow[t]{3}{*}{$\mathrm{RI}+\mathrm{RS}+\mathrm{ME}$} & 1 & $25(0.44)$ & $25(0.56)$ & $25(0.62)$ & $24(0.75)$ & $2(1.00)$ \\
\hline & 10 & $25(0.27)$ & $25(0.34)$ & $25(0.38)$ & $25(0.46)$ & $16(0.91)$ \\
\hline & 100 & $25(0.24)$ & $25(0.30)$ & $25(0.33)$ & $25(0.40)$ & $25(0.81)$ \\
\hline
\end{tabular}

(1) The following covariance parameters are chosen to ensure that $\rho_{1, K}$ varies from 0.1 to 0.9 ; AR $1+\mathrm{ME}: \sigma_{\varepsilon}{ }^{2}=0.91, \sigma_{\varepsilon}{ }^{2}=0.09, \rho_{\varepsilon}=0.11(0.22) 0.99 ;$ RI $+\mathrm{RS}+\mathrm{ME}: \sigma_{0}{ }^{2}=0.7, \sigma_{1}{ }^{2}=0.1, \sigma_{01}=$ $-0.1, \sigma_{e}{ }^{2}=5.3500,1.3510,0.5510,0.2086,0.0185$.

(2) Correlation between two successive measures $p_{j, j+1}$ approaches a maximum $=0.91$ (AR1 $1+M E$ ) if the time-lag between two successive measures goes to zero, i.e. $K$ goes to infinity, irrespective of the error correlation $\rho_{\mathrm{b}}$.

\subsubsection{Resulis}

Tables $4.3-4.5$ show that in case the covariance structure includes auto-correlation, i.e. AR 1 and AR1+ME, two repeated measures per subject are almost always optimal for this cost function. Further, if $K_{\text {opt }}>2$, two repeated measures per subject are still very efficient (relative efficiency of two versus $K_{\text {opt }}$ measures exceeds 0.9 ), except in some extreme cases where the costs per subject $C_{1}$ and/or $C_{2}$ are much larger than the cost $C_{3}$ per repeated measurement and $\rho_{1, K}=0.9$. Note that if $\rho_{1, K}=0.9$, an AR1+ME structure resembles a CS structure. For CS and RI+RS+ME structures, two repeated measures are optimal or highly efficient when the costs per subject in treatment $C_{1}$ and control groups $C_{2}$ are not much larger than the cost $C_{3}$ per repeated measurement, i.e. either $C_{1} \approx C_{2} \approx C_{3}$, or $C_{1} \approx 10 C_{3}$ and $C_{2} \approx C_{3}$. For a RI+RS+ME structure, two repeated measures are also optimal or highly efficient when the correlation $\rho_{1, K}=0.9$ or $C_{1} \approx C_{2} \approx 10 C_{3}$ and $\rho_{1 . K}>0.3$. Otherwise, more than two repeated measures are required to obtain efficient treatment effect estimators. The variance of the GLS 


\section{Chapter 4}

treatment effect estimator can be reduced by more than $50 \%$ by increasing $K$ from 2 to 25 measures per subject if the covariance structure is RI+RS+ME with $\rho_{1, K}<0.9$ or CS and $C$ ? $C_{2} \approx 100 C_{3}$

In conclusion, if the covariance structure includes a first-order auto-regression part, two repeated measures per subject yield efficient treatment effect estimators for the used cost functions. But for a compound symmetric, or a random intercept and random slope plus measurement error covariance structure, more than two repeated measures may be required to obtain this goal. These results agree with our conclusions in Section 4.3, i.e. for a fixed number of subjects and a CS or RI+RS+ME $\left(\rho_{1 . K}<0.9\right)$ structure, the relative efficiency of two versus $K$ repeated measures is relatively small (see Figures $4.2 \mathrm{a}$ and $4.2 \mathrm{~d}$ ).

\subsection{Examples: optimal designs and misspecification of the covariance structure}

In this section, we return to the three examples that were introduced in Section 4.1 and discuss the optimal designs for these examples. In the first example (Diggle, 1990), rats were assigned at random to three treatment groups, receiving no additive $(N=10)$, thyroxin $(N=7)$ or thiouracil $(N=10)$ to the rat's drinking water. The body weight of the rats were measured at the start and after 1, 2,3 and 4 weeks. The second example is a 4-year randomized clinicall trial in which the effect of docosahexaenoic acid (DHA;N=23) is compared with placebo $(N$ $=21$ ) (Hoffman et al., 2004). The main outcome measure, i.e the full-field cone electroretinogram (ERG), was measured at baseline and at subsequent annual visits. In the third example, 45 hospitalized patients with primary depression were randomized into one of three groups receiving trazodone (a new antidepressant), imipramine (an existing antidepressant) or placebo (Feighner, 1980). The score on the Hamilton Psychiatric Scale for Depression was measured at the start of the experiment and at $1,2,3$ and 4 weeks thereafter. The mean profiles of the Hamilton scores for the trazodone $(N=17)$ and placebo groups $(N=$ 10) showed a linearly divergent treatment effect (Feighner, 1980, p. 252). Although the data analysis methods used by the authors of the second and third example assume a compound symmetric covariance structure, neither of these two papers gives information to check this assumption (Feighner, 1980; Hoffman et al, 2004).

Since the data from the first example is available, the question what the optimal number of repeated measures per subject is for this rat-growth experiment will be discussed in detail in the following sections. In Section 4.5.1, we estimate the covariance structure from the rat-growth data. In Section 4.5.2, this structure is then used to design a new study 
optimally, assuming that the estimated structure approximately holds for the future study. The optimal designs for the other two examples will be briefly discussed in Section 4.5.2, assuming a compound symmetric structure like the authors did. The results from previous sections showed, however, that the optimal design depends heavily on the covariance structure. Misspecification of the covariance structure may lead to an inefficient design and will be discussed in Section 4.5.3.

\subsubsection{Rat-growth experiment}

In this experiment, the body-weights of rats were measured at the start and after 1,2 , 3 , and 4 weeks. Further, since the standard deviation was proportional to the mean, Diggle (1990) applied a log-transformation to the data to achieve a stationary covariance structure, i.e. the variance of $y_{i j}$ and the correlation between two repeated measures with a given timelag do not change over time (e.g. Keene, 1995).

For simplicity, we only use the data of the thiouracil and control groups $(N=10 \mathrm{in}$ both groups). A linearly divergent treatment effect was obtained after log-transformation and the pooled covariance and correlation matrices of these two groups were

$$
\left(\begin{array}{lllll}
.0085 & & & & \\
.0090 & .0129 & & & \\
.0063 & .0093 & .0085 & & \\
.0045 & .0063 & .0075 & .0090 & \\
.0028 & .0039 & .0059 & .0078 & .0088
\end{array}\right) \text { and }\left(\begin{array}{llllll}
1.00 & & & & \\
0.86 & 1.00 & & & \\
0.74 & 0.88 & 1.00 & & \\
0.52 & 0.58 & 0.86 & 1.00 & \\
0.32 & 0.37 & 0.68 & 0.88 & 1.00
\end{array}\right)
$$

respectively (Diggle, 1990). The variances and correlations between two repeated measures with a given time-lag showed no systematic increase or decrease over time. Further, the correlation between two repeated measures decreased with increasing time-lag. Therefore, a mixed effects model should incorporate a linearly divergent treatment effect and a stationary covariance structure that allows for a decreasing correlation with increasing time-lagg, which rules out $\mathrm{CS}(\mathrm{RI}+\mathrm{ME})$ and $\mathrm{RI}+\mathrm{RS}+\mathrm{ME}$. 


\section{Chapter 4}

Table 4.6 Body weight data. Treatment effect and standard error estimates (model-based, Kenward and Roger"s (KR) adjusted model-based and empirical) for two and five repeated measures and different covariance structures.

\begin{tabular}{|c|c|c|c|c|c|c|c|c|c|}
\hline \multirow[b]{2}{*}{$K$} & \multirow[b]{2}{*}{$\begin{array}{c}\text { Covariance } \\
\text { structure }\end{array}$} & \multirow[b]{2}{*}{$\hat{\sigma}\left(y_{i j}\right)$} & \multirow[b]{2}{*}{$\hat{\rho}_{1, K}$} & \multirow[b]{2}{*}{$-2 \mathrm{LL}^{(1)}$} & \multirow[b]{2}{*}{$\begin{array}{l}\text { cov } \\
\text { par }\end{array}$} & \multirow[b]{2}{*}{$\hat{\beta}^{\text {CLS }}$} & \multicolumn{3}{|c|}{$\operatorname{SE}\left(\hat{\beta}^{G L s}\right)$} \\
\hline & & & & & & & Model & $\mathbf{K R}$ & Empirical \\
\hline 2 & & .093 & 323 & -65.1 & 2 & .262 & .039 & .041 & .038 \\
\hline \multirow[t]{5}{*}{5} & AR1 & .095 & .526 & -256.6 & 2 & .263 & .036 & .037 & .039 \\
\hline & $\mathrm{AR} 1+\mathrm{ME}$ & .094 & .345 & -263.1 & 3 & .257 & .039 & .040 & .037 \\
\hline & $R I+\left.A R\right|^{(2)}$ & - & - & - & - & - & - & - & - \\
\hline & $\mathrm{RI}+\mathrm{AR} 1+\mathrm{ME}$ & .094 & .272 & -263.3 & 4 & .257 & .040 & .052 & .031 \\
\hline & Toeplitz & .094 & .293 & -263.6 & 5 & .254 & .039 & .043 & .037 \\
\hline
\end{tabular}

(1) The negative -2LL is a matter of an additive constant; the chi-square statistic from the likelihood ratio test is still valid.

Convergence problems occurred under RI+ARI.

The linear mixed effects model (equations 4.1 and 4.2) was fitted to the data assuming different stationary covariance structures that allow for auto-correlation. As a reference structure, Toeplitz was also fitted to the data, because Toeplitz is the most general stationary covariance structure. Results show, however, that Toeplitz did not fit the data better than AR 1+ME or RI+ARI+ME (see Table 4.6).

In Table 4.6, the treatment effect and standard emor estimates are given for AR1, ARI+ME, RI+ARI+ME and Toeplitz applied to the whole body-weight dataset. In addition, a covariance structure with a constant variance was applied to the first and last measures only. Table 4.6 shows that if each rat had only been measured twice, i.e. at the start and at the end of the study, similar treatment effect estimates and model-based standard errors would have been obtained as for five repeated measures per subject, irrespective whether the covariance structure was Toeplitz, AR1, ARI+ME or RI+AR 1+ME. The same conclusion holds for the robust or empirical standard errors (e.g. Verbeke \& Molenberghs, 2000), which are less sensitive than the model-based ones to model misspecification of the covariance structure, and for the Kenward and Roger's (1997) adjusted model-based standard errors, which correct for the fact that covariance parameters are unknown and have to be estimated. 
Estimation problems can occur with the covariance structures in case of more than two repeated measures. In the rat-growth example, the correlation between first and last measures was estimated much higher under AR1 than under the other covariance structures, which resulted in a slightly lower model-based standard error estimate of the GLS treatment effect estimator (see Table 4.6). Further, convergence problems occurred under RI+ARI and a negative measurement error variance estimate was obtained upon fitting AR1+ME or $\mathrm{RI}+\mathrm{AR} 1+\mathrm{ME}$ to the data, because the correlation decreased more rapidly with increasing time-lag than AR1 (see correlation matrix and Figure 4.1). This negative variance estimate may seem a problem in planning studies with many repeated measures, because it implies that as the time-lag becomes smaller, the correlation between two successive measures may become larger than 1. However, the design with two repeated measures is highly efficient in case of $\mathrm{AR} 1$ and in the present case of a rapidly decreasing correlation, and so there is no need to plan many repeated measures in case of this covariance structure.

\subsubsection{Optimal design}

If this rat-growth study were used to plan a future study, two repeated measures would seem to be the best choice, since more than two repeated measures do not decrease the variance of the treatment effect estimator. Note that this is in line with our results in Section 4.4 , i.e. in case the covariance structure includes a first-order auto-regression part, two repeated measures per subject yield highly efficient treatment effect estimators.

Of course, since measuring the body-weight of rats is not expensive, one or two intermediate measures may be added 1) to check the assumption of linearly divergent treatment effects, 2) to check whether the estimated covariance structure holds and 3) to be more robust against drop-out (e.g. Galbraith \& Marschner, 2002; Maxwell, 1998).

For the other two examples (Feighner, 1980; Hoffman et al., 2004) the costs for measuring the main outcome variable are higher and adding intermediate measures will be at the expense of the number of subjects (see Section 4.4). Let us assume with the authors that a compound symmetric (CS) covariance structure is a plausible structure for the outcome variable in both studies. A linear cost function (4.5) will be assumed, where the cost per subject is the same in each treatment group, i.e. the cost ratio $C_{1} / C_{2}=1$, and the cost per subject $\left(C_{1}, C_{2}\right)$ is ten times the cost per repeated measure $C_{3}$, i.e. $C_{2} / C_{3}=10$ As an example, the correlation $\rho_{1, K}$ between any two measures is assumed to be 0.5 . Table 4.3 shows that for 
this cost function and a fixed study budget, the optimal number of repeated measures $K_{\text {opt }}=$ 25.

For the ERG trial (Hoffman et al, 2004), the relative efficiency of the used design ( $K$ $=5, N=44)$ versus the optimal design $\left(K_{\text {opt }}=25, N_{\text {opt }}=18\right)$ can then be shown to be 0.81 . In other words; if the covariance structure is compound symmetric with $\rho_{1, k}=0.5$, the used design with five equally spaced measures requires $(1 / 0.81) \times 44=54$ subjects to obtain the same variance as the optimal design with twenty-five equally spaced measures and 18 subjects. For the depression trial (Feighner, 1980), the relative efficiency of the used $(K=5, N$ $=27)$ versus optimal design $\left(K_{\text {opt }}=25, N_{\text {opt }}=11\right)$ can be shown to be 0.77 .

\subsubsection{Robusthess against misspecification of the covariance structure}

The covariance structure is generally unknown at the design stage of a study and misspecification of the covariance structure may lead to inefficient treatment effect estimators.

In the rat-growth experiment, we assumed that an AR1 structure holds for a future study. This implies that two repeated measures per subject, i.e. one at the start and one at the end of the study, yield highly efficient treatment effect estimators. If this assumption is violated, however, the design with only two repeated measures may be inefficient. For example, if the correct covariance structure is CS instead of ARI and $\rho_{1, K}=0.3$ (see correlation matrix in Section 4.5.1), the relative efficiency of 2 versus 5 repeated measures is about 0.75 , assuming a fixed number of subjects $N$ (see Figure $4.2 \mathrm{a}$ ). This means that due to misspecification, the loss in efficiency is about $25 \%$.

For the other two examples (Feighner, 1980; Hoffman et al., 2004), the authors assumed a CS structure. If this assumption is violated, e.g. the correct structure is AR1, the costs of intermediate measures could be saved and the money could be used for adding more subjects to gain efficiency. For example, for a linear cost function with cost ratios $C_{1} / C_{2}=1$ and $C_{2} / C_{3}=10$, if the correct structure is ARI instead of CS and $\rho_{1, K}=0.5$, the relative efficiency of 5 versus 2 repeated measures can be shown to be 0.86 .

\subsection{Discussion and conclusions}

In the present paper, the relative efficiency of designs with two versus $K$ repeated measures was defined as the inverse of the variances of the generalized least squares (GLS) 
treatment effect estimators. This relative efficiency was discussed for different covariance structures, assuming either a fixed number of subjects or a fixed total budget.

We have shown that two repeated measures often yield highly efficient treatment effect estimators. Adding intermediate measures only reduces the variance in some special cases for which the following rules of thumb may be used in practice. Assuming a fixed number of subjects, efficiency can be gained by adding intermediate measures, if a) the covariance structure is compound symmetric or b) the structure approaches compound symmetry and the correlation between two repeated measures does not exceed 0.80 or $\mathrm{c}$ ) the correlation does not exceed 0.60 , if the time-lag goes to zero. Assuming a fixed total budget, efficiency can be gained by adding intermediate measures at the expense of subjects, if the covariance structure does not include a first-order auto-regression part and adding a subject costs, say, ten times or more than adding an intermediate measure per subject.

The results from Figure 4.2 and Tables 4.3 - 4.5 show that the effect of adding intermediate measures (at the expense of subjects) depends on the covariance structure, which is generally unknown at the design stage of a study. Misspecification of the covariance structure may generally lead to an inefficient design as we have shown for the examples. Since pilot or previous studies may be utilized to estimate the covariance structure and so reduce the risk of misspecification, researchers should report information on the covariance structure that was found. In practice, if the covariance structure is unknown, the optimal design based on a structure between compound symmetry (CS) and first-order auto-regression (AR1), like an AR1 structure with measurement error (AR 1+ME), is preferable in terms of robustness against misspecification of the covariance structure.

Other covariance structures can be obtained in a linear mixed effects model based on random effects, a component of auto-correlation and/or measurement error, like a random intercept and slope model with first-order auto-conelation, but these ran into analytical derivation problems. Further, equidistant time-points are assumed in the present paper. Although this is often a reasonable assumption in planning studies, it is not always efficient to choose equidistant time-points (e.g. Ouwens, Tan \& Berger, 2002; Tan \& Berger, 1999). Furthermore, missing data are often encountered in repeated measures studies. Especially in case of dropout, more than two repeated measures may be required to obtain efficient treatment effect estimators (Galbraith \& Marschner, 2002; Maxwell, 1998). More specifically, Galbraith and Marschner (2002) showed that for the drop-out processes covered in their paper, the effects of drop-out on the power can be large, but when the number of repeated measures $K$ is greater than 2 , the power loss for a given drop-out process is approximately the 


\section{Chapter 4}

same for different values of $K>2$. Thus, in practice, if a design with two repeated measures is highly efficient, one or two intermediate measures may be added 1) to be more robust against drop-out, 2) to check the assumption of linearly divergent treatment effects and 3) to check whether the assumed covariance structure holds.

\section{References}

Bloch, D. A. (1986). Sample size requirements and the cost of a randomized clinical trial with repeated measurements, Slatistics in Medicine, 5, 663-667.

Diggle, P. J. (1990). Time Series: A Biostatistical Introduction, Oxford: Clarendon Press.

Diggle, P. J., Liang, K-Y., \& Zeger, S. L. (1996). Anatysis of Langindinal Data. Oxford: Oxford University Press.

Feighner, J. P. (1980). Trazodone, a triazolopyridine derivative, in primary depressive disorder, ,oumal of Clinical Psychiatry, 41, 250 - 255.

Galbraith, S., \& Marschner, 1. C. (2002). Guidelines for the design of clinical trials with longitudinal outcomes, Confrolled Clinical Trials, 23, 257-273.

Hedeker, D., Gibbons, R. D., \& Waternaux, C. (1999). Sample size estimation for longitudinal designs and attrition: comparing time-related contrasts between two groups, Journal of Educational and Behavioural Statistics, 24, 70-93.

Hoffman, D. R., Locke, K. G., Wheaton, D. H., Fish, G. E., Spencer, R., Birch, D. G. (2004). A randomized, placebo-controlled clinical trial of docosahexaenoic acid supplementation for X-linked retinitis pigmentosa, American Journal of Ophthalmology, 137, 704-718.

Jung, S-H. \& Ahn, C. (2003). Sample size estimation for GEE method for comparing slopes in repeated measurements data, Statistics in Medicine, 22, 1305-1315.

Keene, O. N. (1995). The log transformation is special, Shatistics in Medicine, 14, 81 1-819.

Kenward, M. G., \& Roger, J. H. (1997). Small sample inference for fixed effects from restricted maximum likelihood, Biometrics, 53, 983-997.

Laird, N. M., \& Wang, F. (1990). Estimating rates of change in randomized clinical trials, Controlled Climical Trials, 11, 405-419.

Lui, K-J., \& Cumberland, W. G. (1992). Sample size requirement for repeated measurements in continuous data, Staristics in Medicine $11,633-641$.

Maxwell, S. E. (1998). Longitudinal designs in randomized group comparisons: when will intermediate observations increase statistical power? Psychological Methods, 3, 275290 . 
Moerbeek, M., van Breukelen, G. J. P., \& Berger, M. P. F. (2000). Design issues for experiments in multilevel populations, Journal of Edicational and Behavioural Statistics, 25, 271-284.

Ouwens, M. J. N. M., Tan, F. E. S., Berger, M. P. F. (2002). Maximin D-optimal designs for longitudinal mixed effects models, Biometrics, 58, 735-741.

Overall, J. E., \& Doyle, S. R. (1994). Estimating sample sizes for repeated measurement designs, Controlled Clinical Trials, 15, 100-123.

Overall, J. E., \& Tonidandel, S. (2002). Measuring change in controlled longitudinal studies, Britisch Joumal of Mathematical and Statistical Psychology, 55, 109-124.

Tan, F. E. S., \& Berger, M. P. F. (1999). Optimal allocation of time points for the random effects model. Communications in Statistics - Simulations and Computations, 28, 517 540 .

Verbeke, G., \& Molenberghs, G. (2000). Linear Mixed Models for Longitudinal Data. New York: Springer-Verlag.

Yi, Q., \& Panzarella, T. (2002). Estimating sample size for tests on trends across repeated measurements with missing data based on the interaction term in a mixed model, Controlled Clinical Trials, 23, 481-496. 


\section{Appendix}

A1) The variance of the GLS treatment effect estimator for a random intercept plus ARI $(R I+A R I)$ or random intercept and random slope plus measurement error $(R I+R S+M E)$ covariance structure.

The fomula for the variance of the GLS treatment effect estimator for a RI+AR1 or $\mathrm{RH}+\mathrm{RS}+\mathrm{ME}$ covariance structure is obtained by the following steps:

1 the wariance of the GLS treatment effect estimator is analytically calculated for $K=2$, $3,4,5$ and 6 (see below for example RI+ARI with $K=4$ )

2. similarities of these analytical expressions are generalized to $K$ repeated measures,

3. a general expression is formulated,

4. the general expression is analytically checked for $K=7,8,9$ and 10 ,

5. the general expression is numerically checked for several covariance parameters and $K$ $>11$.

Step 1: In case of a RI+AR I covariance structure and $K=4$, model (4.1) can be reduced to

$$
\begin{aligned}
& y_{i}=X_{i} \beta+Z_{i} b_{i}+\varepsilon_{i} \\
&\left(\begin{array}{l}
y_{i 1} \\
y_{i 2} \\
y_{i 3} \\
y_{i 4}
\end{array}\right)=\left(\begin{array}{ccccc}
1 & 0 & 0 & 0 & 0 \\
0 & 1 & 0 & 0 & G_{i} \times 1 / 3 \\
0 & 0 & 1 & 0 & G_{i} \times 2 / 3 \\
0 & 0 & 0 & 1 & G_{i}
\end{array}\right)\left(\begin{array}{l}
\mu_{1} \\
\mu_{2} \\
\mu_{3} \\
\mu_{4} \\
\beta
\end{array}\right)+\left(\begin{array}{l}
1 \\
1 \\
1 \\
1
\end{array}\right)\left(b_{0 i}\right)+\left(\begin{array}{l}
\varepsilon_{i 1} \\
\varepsilon_{i 2} \\
\varepsilon_{i 3} \\
\varepsilon_{i 4}
\end{array}\right),
\end{aligned}
$$

where $b_{0, i}$ is normally distributed with mean 0 and variance $\sigma_{0}{ }^{2}$. In addition, $c_{i}$ is independent of $b_{0 r}$ and normally distributed with mean vector $\theta$ and covariance matrix $\sigma_{\varepsilon}{ }^{2} \boldsymbol{H}$, where the correlation $\rho_{k:}{ }^{t}$ between $\varepsilon_{j j}$ and $\varepsilon_{i j^{n}}$ decreases with increasing time-lag $\tau=j j-j \mid / 3$. For the linear mixed model in general, the generalized least squares (GLS) estimator of $\beta$ and its variance are given in equation (4.3) and (4.4), respectively. The resulting covariance matrix $V_{i}^{\prime}=Z_{j} D Z_{i}^{\prime}$ $+\sigma_{0}{ }^{2} H$ includes a constant variance $\sigma_{0}{ }^{2}+\sigma_{s}{ }^{2}$ and a covariance between $y_{i j}$ and $y_{i j}$ that decreases with increasing time-lag $\tau=j-j^{7} / 3, \sigma_{0}{ }^{2}+\rho_{s}{ }^{5} \sigma_{s}{ }^{2}$. Since this covariance matrix is homogeneous across subjects, i.e. $\boldsymbol{V}_{i}=\boldsymbol{V}^{r}$ for all $i$, $\sum_{i} \boldsymbol{X}_{i}^{\prime} \boldsymbol{V}_{i}^{+1} \boldsymbol{X}_{i}=N_{C} \boldsymbol{X}_{C}{ }^{\prime} \boldsymbol{V}^{-1} \boldsymbol{X}_{C}+N_{T} \boldsymbol{X}_{T}^{\prime} \boldsymbol{V}^{-1} \boldsymbol{X}_{T}$, where $\boldsymbol{X}_{C}$ and $\boldsymbol{X}_{T}$ are the design matrices $\boldsymbol{X}_{i}$ 
for control $\left(G_{i}=0\right)$ and treatment group $\left(G_{i}=1\right)$, respectively. The model-based variance of the GLS treatment effect estimator can then be calculated using a computer program,

$$
\begin{aligned}
\operatorname{Var}\left(\hat{\beta}_{\mathrm{RLARl}}^{\mathrm{GLS}}\right)= & \frac{N_{i}+N_{c}}{N_{t} N_{o}}\left(1-\rho_{s}^{2}\right) \sigma_{s}^{z} \\
& \times \frac{36\left\{\left(1+\rho_{s}\right) \sigma_{s}^{2}+\left(4-2 \rho_{s}\right) \sigma_{0}^{2}\right\}}{2\left(1+\rho_{s}\right)\left(10 \rho_{s}^{2}-32 \rho_{c}+28\right) \sigma_{s}^{2}+\left(-4 \rho_{s}^{3}+28 \rho_{s}^{2}-80 \rho_{s}+80\right) \sigma_{0}^{2}}
\end{aligned}
$$

Remark.

The variance formulae for $\mathrm{AR} 1+\mathrm{ME}$ and $\mathrm{RI}+\mathrm{AR} 1+\mathrm{ME}$ covariance structures yielded complex expressions for $K=3,4$ or 5 repeated measures. Therefore, the variance formulae could not be generalized to $K$ repeated measures. We could only show that the variance formulae were of the form:

$$
\frac{N_{t}+N_{\mathrm{e}}}{N_{\mathrm{t}} N_{\mathrm{c}}} \times g(K, V)
$$

where $g$ was a complicated function of the number of repeated measures $K$ and the parameters of the covariance matrix $\boldsymbol{V}$.

\section{A2) Variance, covariance and correlation between two repeated measures in a random intercept and slope (RI+RS+ME) model.}

The variances of $y_{i j}$ and covariances between $y_{i j}$ and $y_{i f}$ in a RI+RS +ME model are

$$
\begin{aligned}
\operatorname{Var}\left(y_{i j}\right) & =\sigma_{0}^{2}+\frac{2(j-1)}{K-1} \sigma_{01}+\frac{(j-1)^{2}}{(K-1)^{2}} \sigma_{1}^{2}+\sigma_{i}^{2}, \\
\operatorname{Cov}\left(y_{i j}, y_{i j}\right) & =\sigma_{0}^{2}+\frac{j+j^{\prime}-2}{K-1} \sigma_{01}+\frac{(j-1)\left(j^{\prime}-1\right)}{(K-1)^{2}} \sigma_{1}^{2} .
\end{aligned}
$$

Thus, the correlation $\rho_{j, j^{\prime}}$ between $y_{i j}$ and $y_{i j^{\prime}}\left(j_{,} j^{\prime}=1,2, \ldots, K\right)$ is 


$$
\rho_{j, j}=\frac{\sigma_{0}^{2}+\frac{j+j^{\prime}-2}{K-1} \sigma_{01}+\frac{(j-1)\left(j^{\prime}-1\right)}{(K-1)^{2}} \sigma_{1}^{2}}{\sqrt{\left(\sigma_{0}^{2}+\frac{2(j-1)}{K-1} \sigma_{01}+\frac{(j-1)^{2}}{(K-1)^{2}} \sigma_{1}^{2}+\sigma_{e}^{2}\right)\left(\sigma_{0}^{2}+\frac{2\left(j^{\prime}-1\right)}{K-1} \sigma_{01}+\frac{\left(j^{\prime}-1\right)^{2}}{(K-1)^{2}} \sigma_{1}^{2}+\sigma_{e}^{2}\right)}}
$$

which is non-stationary and decreasing with increasing $\sigma_{e}{ }^{3}$. If the time-lag between $y_{i f}$ and $y_{i f}$ approaches 0 , this correlation does not approach 1 , but is bound to a maximum, especially for large $\sigma_{e}{ }^{2}$. If $\sigma_{01}$ and $\sigma_{1}^{2}$ are very small compared with $\sigma_{0}{ }^{2}$ and $\sigma_{e}{ }^{2}$, the variances and correlations are almost stationary and $\mathrm{RI}+\mathrm{RS}+\mathrm{ME}$ approaches $\mathrm{CS}$. 


\section{Optimal time-points in clinical trials with linearly} divergent treatment effects

This chapter has been accepted for publication in Statistics in Medicine with co-authors H. J. A. Schouten, G. J. P. van Breukelen and M. P. F. Berger 


\section{Q... Abstract}

In repeated measures studies, equidistant time-points do not always yield efficient treatment effect estimators. In the present paper, the optimal allocation of time-points is calculated for a small number of repeated measures, different covariance structures and linearly divergent treatment effects. The gain in efficiency of the treatment effect estimator by using optimally allocated time-points instead of equidistant time-points or by adding optimally spaced measures (at the expense of patients) is then computed. The assumed covariance structure is crucial for the results. For a compound symmetric covariance structure, a large gain in efficiency is obtained by adding repeated measures at the end of the study. For a first-order auto-regressive covariance structure, highly efficient treatment effect estimators are obtained with only two repeated measures, i.e. at the start and at the end of the stidy. For a first-order auto-regressive covariance structure including measurement error, the gain in efficiency by adding optimally spaced measures depends on the covariance parameter values. The gain in efficiency is similar with or without a random intercept. For a fixed study budget, the commonly used design with more than two equally spaced measures was never optimal for the linear cost function and covariance structures that were used. If the covariance structure is unknown, the optimal design based on a first-order auto-regressive covariance structure with measurement error is preferable in terms of robustness against misspecification of the covariance structure. The numerical results are illustrated by two examples.

Keywords: Repeated Measures; Allocation of Time-points; Linearly Divergence, Compound Symmetry; First-order Auto-regression. 


\section{Ophinal time-points}

\subsection{Introduction}

In repeated measures studies, researchers have to make decisions about the number of patients and the number of repeated measures per patient. Sample size formulas for repeated measures studies are given by several authors (e.g. Bloch, 1986; Jung \& Ahn, 2003; Liu, 2003; Lui \& Cumberiand, 1992; Schouten, 1999; Yi \& Panzarella, 2002). The required number of patients to obtain efficient treatment effect estimators, however, decreases as the number of repeated measures increases (e.g. Arndt, Jorge, Turvey \& Robinson, 2000; Maxwell, 1998; Raudenbush \& Liu, 2001; Schlesselman, 1973; Vickers, 2003; Yi \& Panzarella, 2002). For a specified cost function, the combination of number of repeated measures and sample sizes that minimizes this function for given power can be calculated (e.g. Bloch, 1986; Lui \& Cumberland, 1992). Usually, repeated measures are assumed to be equidistant, but this does not always yield efficient treatment effect estimators (e.g. Ouwens, Tan \& Berger, 2003; Tan \& Berger, 1999). The decisions concerning sample size, number of repeated measures and allocation of time-points depend on the covariance structure of the repeated measures and on the pattern of treatment effect over time (e.g. Overall \& Doyle, 1994). In most repeated measures studies, a compound symmetric and/or a first-order autoregressive covariance structure is assumed.

In the present paper, the optimal allocation of time-points for the generalized least squares (GLS) estimator of a linearly divergent treatment effect is calculated for several covariance structures. A linearly divergent treatment effect means that trends within the groups may be curvilinear, but that the expected difference between the groups increases linearly over time, possibly after a data transformation. This type of pattern can often be anticipated in clinical trials with a relatively short duration in which a maximum treatunent effect has not yet been achieved (e.g. Owerall \& Doyle, 1994). Several examples of linearly divergent treatment effects can be found in the literature (e.g. Diggle, 1990, p. 8; Feighner, 1980; Frison \& Pocock, 1994; Furberg, et al., 1994; Gruttola, Lange \& Dafni, 1991 ; Jennrich \& Schluchter, 1986; Raudenbush \& Chan, 1993).

In the present paper, two questions are dealt with. The first question concerns the gain in efficiency when an optimal allocation of time-points is used instead of equidistant timepoints. The second question is whether two repeated measures are sufficient to obtain highly efficient treatment effect estimators or whether optimally spaced measures need to be added to obtain this goal. 
We will restrict the number of repeated measures to a maximum of five, because the results are expected to be similar for six or more repeated measures and the benefit of adding one extra measure to a series of repeated measures decreases rapidly with its length (e.g. Vickers, 2003). Further, it has been shown that in case of equidistant time-points, two up to five repeated measures yield efficient treatment effect estimators for many covariance structures (Arndt; Jorge, Turvey \& Robinson, 2000):

In Section 5.2, the random effects model is introduced and different covariance structures are discussed. In Section 5.3, the optimal allocation of time-points is calculated and the variance of the treatment effect estimator for optimally allocated time-points is compared with its variance for equidistant time-points. In Section 5.4 , the gain in efficiency when optimally spaced measures are added to a design with two repeated measures only is discussed. Misspecification of the covariance structure is discussed in Section 5.5. The numerucal results are illustrated by two examples in Section 5.6. Finally, the conclusions are summarized and discussed in Section 5.7.

\subsection{Random effects model}

\subsubsection{Model}

For patient $i$, let $y_{i j}$ be the $j$ th repeated measurement of a continuous outcome variable measured at time $t_{j}(i=1,2, \ldots, N ; j=1,2, \ldots, K)$. For simplicity, the time-points are assumed to be the same for each patient. Further, the first measure for each patient is taken at $t_{1}=0$, i.e. just before randomization, and the total duration of the treatment period is fixed and rescaled to unity, i.e. $t_{K}=1$. For patient $i$, a random effects model (e.g. Diggle, Liang \& Zeger, 1996, p. 87) can be written as

$$
y_{i}=X_{i} p+Z_{i} b_{i}+\varepsilon_{i}+e_{i}
$$

where

$$
\boldsymbol{X}_{i} \beta=\left(\begin{array}{ccccc}
1 & 0 & \cdots & 0 & G_{i} \times t_{1} \\
0 & \ddots & \ddots & \vdots & \vdots \\
\vdots & \ddots & \ddots & 0 & \vdots \\
0 & \cdots & 0 & 1 & G_{i} \times t_{K}
\end{array}\right)\left(\begin{array}{c}
\mu_{i} \\
\vdots \\
\mu_{K} \\
\beta
\end{array}\right) \text { and } Z_{i} b_{i}=\left(\begin{array}{c}
1 \\
\vdots \\
1
\end{array}\right)\left(b_{0 i}\right)
$$


Here $\beta$ is the treatment effect parameter and $G_{i l}$ is an indicator of treatment, i.e. $G_{i}=1$ if patient $i$ belongs to the (active) treatment group and $G_{l}=0$ otherwise. Thus, $y_{l}$ has expectation $\mu_{j}$ in the control group and $\mu_{j}+\beta t_{j}$ in the treatment group. This means that the trend within a group may be of any type, but the group difference increases linearly over tine. Due to randomization, no group effect is assumed at $t_{\mathrm{l}}=0$. Further, $\varepsilon_{i}$ is a component of autocorrelation and $\boldsymbol{e}_{i}$ is an extra component of measurement error. The random effects vector $\boldsymbol{b}_{i}$ and the error vectors $\varepsilon_{i}$ and $e_{i}$ are assumed to be independently, normally distributed with mean vectors 0 and covariance matrices $D, \sigma_{\varepsilon}^{2} \boldsymbol{H}$ and $\sigma_{e}^{2} \boldsymbol{I}_{K}$, respectively, where $\boldsymbol{I}_{K}$ is the $K$ dimensional identity matrix. As a result, the covariance matrix of the outcome vector $y_{i}$ is $\operatorname{Var}\left(\boldsymbol{y}_{i}\right)=\boldsymbol{V}_{i}=\boldsymbol{Z}_{i} \boldsymbol{D} \boldsymbol{Z}_{i}{ }^{\prime}+\sigma_{s}^{2} \boldsymbol{H}+\sigma_{e}{ }^{2} \boldsymbol{I}_{K}$.

Notice that the measurement errors $e_{i j}$ and $e_{i j}$ are assumed to be independent, irrespective of the time-lag $\tau=\left|t_{j}-t_{l}\right|$. If this time-lag is $0_{2}$ this would indicate that the two time-points at which the subjects were measured are extremely close to each other.

In the present paper, a first-order auto-regressive (AR1) correlation structure is considered for $\boldsymbol{H}$, i.e. the correlation $\rho_{\varepsilon}{ }^{x}$ between $\varepsilon_{i j}$ and $\varepsilon_{i j^{\prime}}$ decreases with increasing time-lag $\tau=\left|t_{i}-t_{j}\right|$ and $\rho_{\varepsilon}$ is the correlation between $\varepsilon_{i 1}$ and $\varepsilon_{i K}$.

The only random effect considered here is a random intercept $b_{0 i}$, i.e. $D=\left(\sigma_{0}{ }^{2}\right)$, because quite general covariance structures can be obtained from this random intercept model including auto-correlation and measurement error (e.g. Diggle, Liang \& Zeger, 1996, p. 89).

For simplicity, the covariance matrix $V_{i}$ of $y_{i}$ is assumed to be homogeneous across persons and groups, i.e. $V_{i}=V$ for all $i$.

For the random effects model (5.1), the generalized least squares (GLS) estimator of $\beta$ is

$$
\hat{\boldsymbol{\beta}}^{\mathrm{NHS}}=\left(\sum_{i=1}^{N} \boldsymbol{X}_{\mathrm{i}}^{\prime} \boldsymbol{V}^{\mu 1} \boldsymbol{X}_{i}\right)^{-1} \sum_{i=1}^{N} \boldsymbol{X}_{i}^{\prime \prime} \boldsymbol{V}^{-1} \boldsymbol{y}_{i}
$$

The model-based variance of the GLS estimator of $\beta$ (e.g. Diggle, Liang \& Zeger, 1996, p. $59)$ is

$$
\operatorname{Var}\left(\hat{\beta}^{\text {GLS }}\right)=\left(\sum_{i=1}^{N} X_{i}^{*} V^{-1} X_{i}\right)^{-1}
$$




\section{Chapter 5}

The optimal allocation of time-points $\left[0, t_{2}, \ldots, l_{K-1}, 1\right]$ depends on the specified optimality criterion, which is a function of the variance matrix (5.4). Several optimality criteria are proposed (e.g. Atkinson \& Donev, 1996, p. 106). In the present paper, we will apply the $c$-optimality criterion, i.e. $\operatorname{Var}\left(c^{\prime} \hat{\beta}^{\text {GLS }}\right)$, where $c=(0, \ldots 0,1)^{t}$, because we are only interested in the linearly divergent treatment effect parameter $\beta$. An optimal design has optimal allocation of time-points. These are obtained here by minimizing the $\operatorname{Var}\left(c^{\prime} \hat{\beta}^{\text {cas }}\right)$ over all possible allocations of time-points, where the design space of a time-point is assumed to be $[0,1]$ without loss of generality. Two designs $\xi_{\text {and }} \xi_{2}$ can then be compared by the relative efficiency $e f f\left(\xi_{1} \mid \xi_{2}\right)$ of design 1 versus design 2 , which is defined as

$$
\mathscr{e f f}\left(\xi_{1} \mid \xi_{2}\right)=\frac{\operatorname{Var}\left(\hat{\beta}^{\text {OLs }}\right)_{\xi_{2}}}{\operatorname{Var}\left(\hat{\beta}^{\text {oLs }}\right)_{\xi_{1}}}
$$

\subsubsection{Covariance struchures}

In the present paper, the outcome $y_{i j}$ depends on three sources of random variation: a

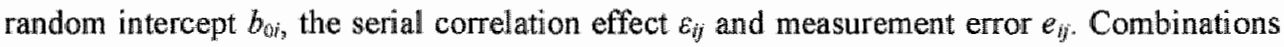
of these three sources yield five well-known covariance structures: only serial correlation (AR1), serial correlation with measurement error (AR1+ME), random intercept with measurement error ( $R I+M E)$, random intercept with serial correlation $(\mathrm{RI}+\mathrm{AR} 1)$, random intercept with serial correlation and measurement error (RI+ARI + ME) (e.g. Diggle, Liang \& Zeger, 1996, p. 79-87). Notice that the random intercept and measurement error (RI+ME) structure yields a compound symmetric (CS) covariance structure, i.e a constant Var $\left(y_{i j}\right)$ over

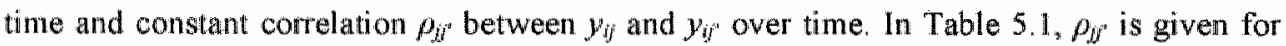
these covariance structures with time-lag $\tau=\left|l_{j}-l_{j}\right|=0$ and 1 . Additionally, $\rho_{j^{n}}$ is given for an infinite time-lag. "The denominator of the correlation is $\operatorname{Var}\left(y_{i j}\right)$. For an AR 1 structure, $\operatorname{Var}\left(y_{i j}\right)$ is equal to $\sigma_{g^{2}}$.

Table 5.1 shows that all covariance structures imply a constant variance over time. Further, except for the CS structure, the correlation $\rho_{y}$ between $y_{i j}$ and $y_{i j^{\prime}}$ decreases with increasing time-lag $t=\left|t_{j}-t_{j}\right|$. For an $\mathrm{ARL}$ structure, the correlation $\rho_{i j^{\prime}}$ decreases relatively fast from 1 , obtained when $\tau=0$, to 0 , obtained for an infinite time-lag. From Table 5.1 it can be seen that a random intercept ensures a correlation greater than 0 and measurement error leads to correlations less than 1. So, the CS and AR 1 structures are the extreme opposites in 
terms of dependence of $\rho_{j j^{*}}$ on the time-lag. Therefore, in the next section, the optimal allocation of time-points is first discussed for these structures.

Table 5.1 Correlation $p_{i j^{\prime}}$ between $y_{i j}$ and $y_{i j^{\prime}}$ for covariance structures based on a random intercept (RI), auto-correlation (AR1) and measurement error (ME).

\section{Correlation $p_{j j^{\prime}}$ between $y_{i j}$ and $y_{i j}$}

Covariance

structure $\quad \tau=\left|t_{j}-t_{j^{*}}\right| \quad \tau=0 \quad t=1 \quad$ infinite time-lag

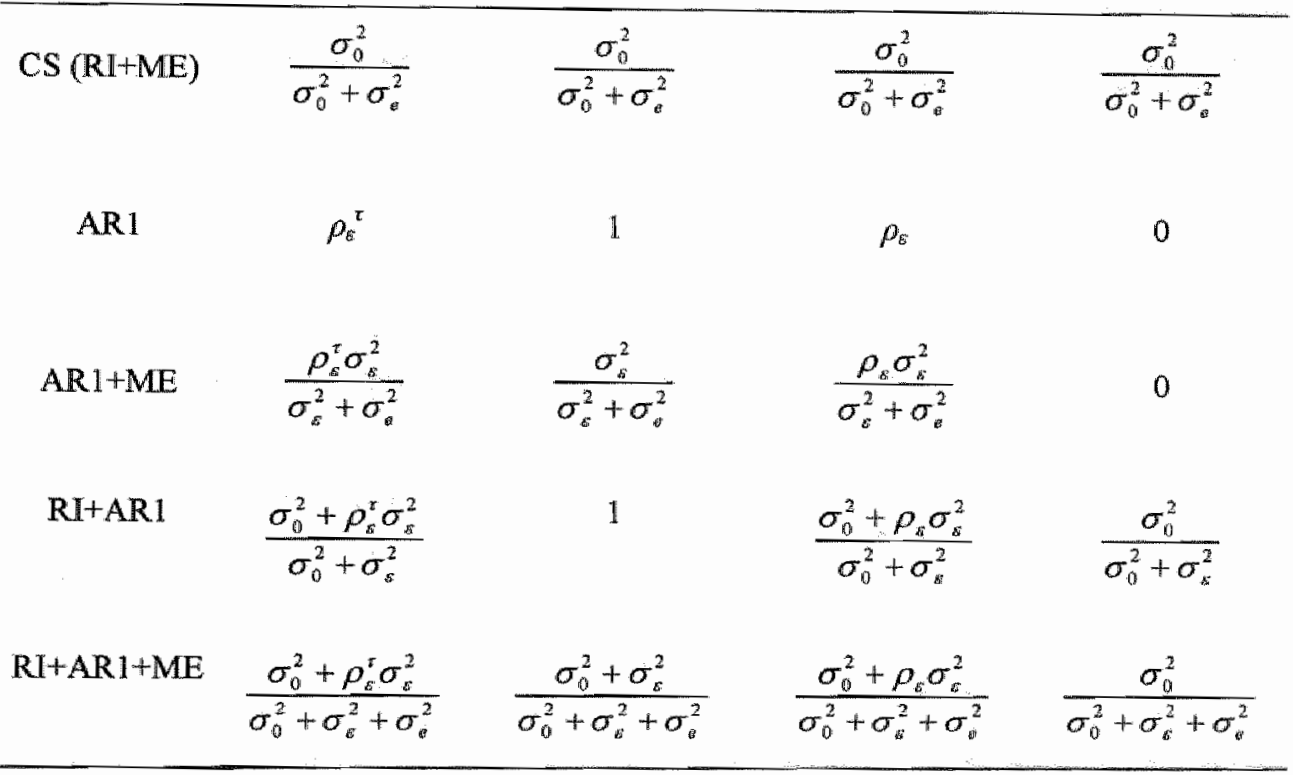

\subsection{Optimal allocation of time-points and relative efficiency of equidistant time-points}

The covariance structure is generally unknown in the design stage of a study. In the following two sections, it will be assumed known. In Section 5.5 , the effect of misspecification of the covariance structure will be discussed.

The optimal allocation of time-points $\left[0, t_{2}, \ldots, t_{k-1}, 1\right]$ in design space $[0,1]$ is calculated by minimizing the variance of the GLS treatment effect estimator Var $\left(c^{\prime \prime} \hat{p}^{c L . s}\right)$. For example, for $K=3$ repeated measures per patient and known covariance structure and parameters, the optimal allocation is abtained by minimizing the variance as a function of $t_{2}$ since $t_{1}=0$ and $t_{3}=1$. This optimal choice for $t_{2}$ was calculated analytically for the CS 
structure with $K=3$ repeated measures (see Appendix), and numerically for the other covariance structures.

\subsubsection{Compound symmetry versus first-order auto-regression}

In Table 5.2 , the optimal allocation of time-points was calculated for compound symmetric (CS) and first-order auto-regressive (AR1) covariance structures and $K=3,4$ or 5 repeated measures, where the first and last time-points were fixed at $t_{1}=0$ and $t_{K}=1$, respectively. The correlation $p_{\mathbb{K} K}$ between first and last measures is taken to be $0.3,0.5$ or 0.7 .

Table 5.2 Optimal allocation of time-points $t_{2}, \ldots, t_{K-1}$ as function of covariance structure and $\rho_{1}$ for $K=3,4$ or 5 .

\section{Correllation $\rho_{\mathbb{K}}$ between first and last measures}

\begin{tabular}{ccccc} 
Cov structure & $K$ & 0.3 & 0.5 & 0.7 \\
\hline CS (- RI+ME) & 3 & 1.00 & 1.00 & 1.00 \\
& 4 & $1.00,1.00$ & $1.00,1.00^{(1)}$ & $0.00,1.00$ \\
& 5 & $1.00,1.00,1.00$ & $0.00,1.00,1.00$ & $0.00,1.00,1.00$ \\
AR1 & 3 & 0.64 & 0.64 & 0.64 \\
& 4 & $0.50,0.78$ & $0.50,0.78$ & $0.50,0.78$ \\
ARI+ME & 5 & $0.42,0.65,0.84$ & $0.42,0.65,0.84$ & $0.42,0.65,0.84$ \\
& 3 & 1.00 & 1.00 & 1.00 \\
& 4 & $1.00,1.00$ & $0.00,1.00$ & $0.00,1.00$
\end{tabular}

(1) Two allocations of time-points $t_{2}, t_{3}$ are optimal: $1.00,1.00$ and $0.00,1.00$

Table 5.2 shows that for a CS structure, the optimal choice for the additional measures is almost always at the end of the study. Notice that for $K=4$ and $\rho_{K}=0.5$, the optimal allocation of $\left(t_{2}, t_{3}\right)$ is $(1.00,1.00)$ as well as $(0.00,1.00)$. For an AR1 structure, the optimal spacing of intermediate measures is shifted to the right compared to equal spacing (see Table 5.2). For example, in case $K=5$, the optimal allocation is $t_{2}=0.42, t_{3}=0.65$ and $t_{4}=0.84$, whereas equidistant time-points imply $t_{2}=0.25, t_{3}=0.50$ and $t_{4}=0.75$. 
Table 5.3 Relative efficiency of equidistant versus optimally allocated time-points as function of covariance structure and $p_{M}$ for $K=3,4$ or 5 .

\begin{tabular}{ccccc}
\hline & \multicolumn{3}{c}{ Correlation $\rho_{i K}$ between first and last measures } \\
Cov structure & $K$ & 0.3 & 0.5 & 0.7 \\
\hline CS (=RI+ME) & 3 & 0.6625 & 0.6875 & 0.7125 \\
& 4 & 0.5852 & 0.6296 & 0.5948 \\
& 5 & 0.5625 & 0.5556 & 0.5392 \\
ARI & 3 & 0.9988 & 0.9998 & 1.0000 \\
& 4 & 0.9993 & 0.9999 & 1.0000 \\
& 5 & 0.9996 & 0.9999 & 1.0000 \\
ARI & & & & 0.8082 \\
& 3 & 0.9096 & 0.8752 & 0.7408 \\
& 4 & 0.8907 & 0.8532 & 0.7116 \\
\hline
\end{tabular}

In Table 5.3, the relative efficiency of equidistant versus optimally allocated timepoints (see equation 5.5) was calculated under the same conditions as in Table 5.2. Table 5.3 shows that, for a CS structure, equidistant time-points do not yield highly efficient treatment effect estimators. If repeated measures are optimally spaced instead of equally spaced, the variance of the GLS treatment effect estimator decreases by about $30 \%$ for $K=3$ and by about $40 \%$ for $K>3$. For an AR1 structure, the relative efficiency of equidistant versus optimally allocated time-points is almost one (see Table 5.3 ).

These results can be explained as follows. For a CS structure, the correlation between two repeated measures is independent of the time-lag between the measures. "The optimal choice for an additional measure is then at the end of the study, where the expected treatment effect is the largest. For an AR1 structure, the correlation between two repeated measures increases with decreasing time-lag between the measures. Thus, for an AR1 structure, the optimal choice for an additional measure is not exactly at the end, because then the correlation between the additional and last measure $\rho_{, k}$ becomes equal to one but it is shifted towards the end compared with equidistant time-points, because of the assumption of a linearly divergent treatment effect. 


\section{Chapter 5}

\subsubsection{Other covariance structures}

When measurement error is added to the model with AR 1 , the maximum correlation can not be equal to 1 , even if the time-lag between repeated measures is 0 (see Table 5.1). As an example, the optimal allocation of time-points is presented in Table 5.2 for the AR1 $\mathrm{ME}$ structure with $\sigma_{\varepsilon}^{2}=0.8$ and $\sigma_{e}^{2}=0.2$, so that the correlation $\rho_{i j}$ between $y_{j j}$ and $y_{i j^{\prime}}$ is always smaller than or equal to 0.8 , even if the time-lag $\tau$ goes to 0 (see Table 5.1). Notice that, without loss of generality, the variance of $y_{i j}$ is fixed at 1 . The relative efficiency of equidistance versus optimal allocation of time-points for this covariance structure is presented in Table 5.3. Similar results have been obtained for the AR $1+M E$ structure with $0.60 \leq \sigma_{s}^{2} \leq$ $0.90\left(0.40 \geq \sigma_{e}^{2} \geq 0.10\right)$ (not shown here).

Table 5.2 shows that the optimal allocation of time-points is similar for the AR $1+\mathrm{ME}$ structure and the CS structure. For an $\mathrm{ARI}+\mathrm{ME}$ structure, the relative efficiency of equidistant versus optimally allocated time-points depends on the correlation $\rho_{\varepsilon}$ between the auto-correlation effects $\varepsilon_{i 1}$ and $\varepsilon_{i k}$ (see Table 5.3). If the correlation $\rho_{\varepsilon}$ approaches 1 , the correlation $\rho_{1}$ between first and last measures approaches the maximum correlation, which is achieved when the time-lag $\tau$ is 0 , and the ARI+ME structure approaches the CS structure. Then equidistant time-points do not yield highly efficient treatment effect estimators (see Table 5.3). If $\rho_{t:}$ is much smaller than 1 , however, the correlation $\rho_{1 K}$ between first and last measures is much smaller than the maximum correlation (see Table 5.1) and highly efficient treatment effect estimators are obtained under equidistant time-points (see Table 5.3).

If the amount of measurement error increases, the maximum correlation decreases (see Table 5.1) and the AR. 1+ME structure approaches the CS structure In that case, equidistant time-points do not yield highly efficient treatment effects (not shown here).

Addition of a random intercept to the model hardly affected the results, i.e similar results were obtained for the $A R 1$ and $R I+A R 1$ structures and likewise for the $A R 1+M E$ and RI+ARI+ME structures. These results are in line with those found by Tan and Berger (1999)

In conclusion, for a compound symmetric (CS) covariance structure, equidistant timepoints do not yield highly efficient treatment effect estimators and the optimal choice for the additional measures is almost always at the end of the study. For AR1 and RI+ARI covariance structures, however, highly efficient treatment effect estimators are obtained under equidistant time-points. For $\mathrm{AR} 1+\mathrm{ME}$ and $\mathrm{RI}+\mathrm{AR} 1+\mathrm{ME}$ covariance structures, the results depend on the amount of measurement error and auto-correlation $\rho_{\beta}$. The relative efficiency of equidistant versus optimally allocated time-points decreases with increasing amount of 


\section{Optimal time-poins}

measurement error and/or increasing auto-correlation $\rho_{\mathrm{S}}$ because then the AR $1+\mathrm{ME}$ and $\mathrm{RI}+\mathrm{ARI}+\mathrm{ME}$ structures approach the CS (=RI+ME) structure.

\subsection{Effect of adding optimally spaced measures}

In this section, the effect of adding optimally spaced measures on the efficiency of the GLS treatment effect estimator will be discussed, where either the number of patients $N$ is fixed (Section 5.4.1) or the budget $C$ for sampling and measuring patients is fixed (Section $5.4 .2)$.

\subsubsection{Fixed number of patients}

To investigate the effect of adding optimally spaced measures, the relative efficiency of 2 versus 3,4 or 5 optimally spaced measures (see equation ( 5.57$)$ was calculated for a fixed number of patients (see Table 5.4). Although the same covariance parameters for the AR1+ME structure will be assumed as in Tables 5.2 and 5.3 , i.e. $\sigma_{s}{ }^{2}=0.8$ and $\sigma_{e}{ }^{2}=0.2$, similar results were obtained for the ARI $1+\mathrm{ME}$ structure with other values of $\sigma_{\varepsilon}^{2}$ and $\sigma_{a t}{ }^{2}$. These are, however, omitted.

Table 5.4 Relative efficiency of 2 versus 3,4 or 5 optimally allocated time-points as function of covariance structure and $\rho_{1 K}$, where the number of patients $N$ is fixed.

Correlation $\rho_{\mathrm{L} K^{\prime}}$ between first and last measures

\begin{tabular}{ccccc} 
Cov structure & $K$ & 0.3 & 0.5 & 0.7 \\
\hline CS (=RI+ME) & 3 & 0.6154 & 0.6667 & 0.7059 \\
& 4 & 0.4872 & 0.5556 & 0.5363 \\
& 5 & 0.4231 & 0.4444 & 0.4383 \\
AR1 & 3 & 0.9885 & 0.9980 & 0.9998 \\
& 4 & 0.9863 & 0.9976 & 0.9998 \\
& 5 & 0.9856 & 0.9975 & 0.9998 \\
AR1+ME & 3 & 0.8901 & 0.8667 & 0.8039 \\
& 4 & 0.8535 & 0.8222 & 0.6972 \\
& 5 & 0.8352 & 0.7852 & 0.6318 \\
\hline
\end{tabular}


Table 5.4 shows that for a fixed number of patients and $\mathrm{CS}(=\mathrm{RI}+\mathrm{ME})$ structure, the benefit of adding a small number of optimally allocated measures is substantial. More specifically, if one, two or three optimally spaced measures are added to a two repeated measures design, while the number of patients is fixed and the covariance structure is $\mathrm{CS}$, the variance of the treatment effect estimator decreases by about $35 \%, 50 \%$ or $60 \%$, respectively. This means that when the number of repeated measures $K$ increases from 2 to 3 , the variance decreases from $100 \%$ to $65 \%$, i.e. a decrease of $35 \%$. When $K$ increases from 3 to 4 , the variance decreases from $65 \%$ to $50 \%$, i.e. a decrease of $(1-0.50 / 0.65) \times 100 \%=23 \%$. Similarly, when $K$ increases from 4 to 5 , the variance decreases with $(1-0.40 / 0.50) \times 100 \%=$ $20 \%$. This confirms the diminishing effect of adding a repeated measure to a series of repeated measures (e.g. Vickers, 2003).

For an AR1 structure, however, two repeated measures yield treatment effect estimators with almost the same efficiency as those obtained with three, four or five optimally spaced measures (see Table 5.4). For an AR1+ME structure, the benefit in terms of efficiency of adding optimally spaced measures depends on the covariance parameter values. If the correlation $\rho_{1 K}$ between first and last measures approaches the maximum correlation (obtained when the time-lag $\tau$ is 0 ), the AR1+ME structure approaches the CS structure and the relative efficiency of 2 versus 3, 4 or 5 optimally spaced measures decreases (see Table 5.4). Notice that the ARIHME structure also approaches the CS structure when the amount of measurement error increases (see Table 5.1). In that case, the design with two repeated measures does not yield efficient treatment effect estimators (not shown here).

Finally, it should be noted that the addition of a random intercept to the model did not seriously affect the results, i.e. similar results were obtained for the ARI and RI+ARI structures and likewise for the AR 1+ME and RI+AR I +ME structures.

In conclusion, for a fixed number of patients and a CS covariance structure, adding optimally spaced measures to a two repeated measures design will increase the efficiency of the treatment effect estimators. For ARI and RI+ARI covariance structures, however, two repeated measures are sufficient to yield highly efficient treatment effect estimators. For AR1+ME and RI+AR 1+ME, the results depend on their covariance parameters; the relative efficiency decreases with increasing $\sigma_{i}{ }^{2}$ or $\rho_{\varepsilon,}$ i.e. as the covariance structure approaches the CS structure. 


\section{Optimal time-points}

\subsubsection{Fixed budget}

In practice, the addition of patients and repeated measures per patient will increase the costs of the study. Given a fixed total budget, more repeated measures per patient imply that fewer patients can be included into the study and vice versa. For a fixed budget, the optimal combination of sample size $N$ and number of optimally allocated repeated measures $K$ may be obtained by minimizing the variance of the GLS treatment effect estimator $\operatorname{Var}\left(c ; \hat{\beta}^{\text {aLs }}\right)$ subject to a linear cost function,

$$
C=C_{1} N+C_{2} N K
$$

where $C$ is the total budget for sampling and measuring patients, excluding overhead cost, $C_{1}$ is the cost per patient, i.e. cost for recruitment and cost of treatment, and $C_{2}$ is the cost per repeated measure. For simplicity, we will assume that the cost per patient is equal for both treatment and control group. We also assume that the number of repeated measures $K$ is restricted to a maximum of five measures and that sampling a patient will cost more than obtaining a repeated measure per patient, i.e. the cost ratio $R=C_{1} / C_{3}>1$. This means that equation (5.6) can be rewritten as $N=C /\left\{C_{2}(R+K)\right\}$

If the cost function is specified and the covariance structure is known, the optimal combination of sample size $N_{\text {opt }}$ and number of optimally allocated repeated measures $K_{\text {opt }}$ may be computed. As an example, assume a cost ratio $R=10$ (i.e. sampling a patient cost ten times as much as a repeated measure per patient), assume $C=3000 C_{2}$ (i.e. $N=3000 /$ $(10+K))$ and assume that the correlation $\rho_{1 K}$ between first and last measures is 0.5 . We found that the covariance structure is cruciall for the optimal combination of number of repeated measures $K_{\text {opt }}$ and sample size $N_{\text {opt }}, K_{\text {opt }}=5$ and $N_{\text {opt }}=200$ for a CS structure, $K_{\text {opt }}=2$ and $N_{\text {opt }}=250$ for an ARI structure, $K_{\text {opt }}=3$ and $N_{\text {opt }}=230$ for the AR1+ME structure with $\sigma_{i}{ }^{2}=$ 0.2 (and $\sigma_{k}{ }^{2}=0.8$ ) and $K_{\text {opt }}=5$ and $N_{\text {opt }}=200$ for the ARI + ME structure with $\sigma_{6}{ }^{2}=0.4$ (and $\sigma_{8}^{2}=0.6$ ). The optimal allocation of time-points can then be obtained from Table 5.2.

These results show that the optimal combination of sample size $N$ and number of optimally allocated repeated measures $K$ depends heavily on the covariance structure and that a design with more than two equally spaced measures was never optimal for the linear cost function and covariance structures that were used. For an AR1 structure, the costs of adding measures could be saved by measuring each patient only twice (at the start and at the end of 
the study). This money could then be used to include more patients in the study and so gain efficiency. For CS and AR1+ME structures, the efficiency could be increased by using the optimal allocation of the time-points instead of equidistant time-points (see Table 5.3).

\subsection{Misspecification of covariance structure}

The results of previous sections show that the optimal allocation of time-points and the effect of adding optimally spaced measures rely heavily on the assumed covariance structure. The problem is, of course, that this covariance structure is often unknown in the design stage. Although previous or pilot studies may be utilized to estimate the covariance structure, milsspecification of the covariance structure may lead to an inefficient design.

We will now compare the efficiency of the design based on the assumed (incorrect) covariance structure with the one based on the correct covariance structure. For a fair comparison of the efficiencies of two designs, the budget for sampling and measuring patients will be assumed fixed. The number of repeated measures $K$ will be restricted to a maximum of five measures and the linear cost function in equation (5.6) will be assumed, where the cost ratio $R=C_{1} / C_{2}$ is again fixed at 10 , i.e. sampling a patient will cost ten times as much as a repeated measure per patient. For a fixed budget, adding repeated measures will be at the expense of patients. For example, the design based on a CS structure would include more repeated measures per patient and fewer patients than the design based on an AR1 structure (see Section 5.4.2).

Table 5.5 Relative efficiency of the design based on the assumed versus correct covariance structure; cortidation between first and last measures $\rho_{1 K}=0.5$, cost ratio $R=C_{1} / C_{2}=10$, the number of repeated measures $K \leq 5$ and the total budget $C$ is fixed.

\begin{tabular}{|c|c|c|c|}
\hline Assumed $\backslash$ Correct & $\mathrm{CS}$ & ARI & $\mathrm{AR} 1+\mathrm{ME}$ \\
\hline CS & 1 & 0.8000 & 0.9566 \\
\hline ARI & 0.5556 & 1 & 0.9389 \\
\hline $\mathrm{AR} 1+\mathrm{ME}^{(1)}$ & 0.7692 & $0.923 \pi$ & 1 \\
\hline
\end{tabular}

(1) The measurement error variance $\sigma_{v}{ }^{2}=0.2$ and serial error variance $\sigma_{\varepsilon}{ }^{2}=0.8$.

In Table 5.5, the relative efficiency of the design based on the assumed (incorrect) versus correct covariance structure is presented for compound symmetry (CS), first-order 
auto-regression (ARI) and first-order auto-regression with measurement error (ARI+ME) Adding a random intercept to $A R 1$ or $A R I+M E$ did not seriously affect the results. The correlation $\rho_{1 K^{K}}$ between first and last measures is assumed to be 0.5 and, for an $A R I+M E$ structure, the measurement error variance $\sigma_{i}^{2}=0.2$ (and $\sigma_{i}^{2}=0.8$ ), because more measurement error (e.g. $\sigma_{e}{ }^{2}=0.4$ ) would lead to the same optimal design as for a CS stricture (see Section 5.4.2).

Table 5.5 shows that if an optimal design is incorrectly based on a CS structure, while the correct covariance structure is $A R 1$ or AR1+ME, then the loss in efficiency due to misspecification is less than or equal to $20 \%$. However, if an optimal design were incorrectly based on an ARI structure, while the correct covariance structure is $\mathrm{CS}$, the loss in efficiency is about $44 \%$.

\subsection{Examples}

Two examples are given here to illustrate the numerical results of the previous sections. The first example is a randomized trial in which the effects of two different additives (thyroxin and thiouracil) to rats" drinking water were compared (see Diggle, 1990, p. 8). Thirty rats were randomly assigned to one of three treatment groups (control group, thyroxin and thiouracil). The body-weight of each rat was observed at the beginning of the experiment and at $1,2,3$ and 4 weeks later. The mean profiles for log-transformed body-weights between the thiouracil (or thyroxin) and control groups showed a linearly divergent treatment effect (see Diggle, 1990, p. 154). A tirst-order auto-regressive (AR1) covariance structure seems plausible; the correlation between repeated measures decreased from 0.88 to 0.34 when the time-lag increased from 1 week to 4 weeks (see Diggle, 1990, p. 139). Therefore, similar variances of the GLS treatment effect estimators would have been obtained if the repeated measures would have been optimally allocated instead of equally spaced (see Table 5.3). More importantly, the intermediate measures, even with optimal allocation, have a very small contribution to the efficiency of the GLS treatment effect estimator (see Table 5.4). Thus, the costs of adding measures could be saved and the money could be used for adding more rats to gain efficiency. For a linear cost function (5.6), in which sampling rat costs ten times as much as a repeated measure per rat; and with a fixed budget, the number of rats could be increased by $25 \%$ if each rat were measured only twice (see Section 5.4 .2 ). The relative efficiency of the design with five equidistant time-points versus the optimal design with two time-points (one at the start and one at the end of the study) is about 0.8 . In other words, if 
each rat were measured once at the start and once at the end of the study instead of every week, the variance of the GLS rreatment effect estimator would decrease by about $19 \%$, given the same study budget.

The second example is a 4-year randomized clinical trial in which male patients with X-linked retinitis pigmentosa (XLRP) received low docosahexaenoic acid (DHA) or placebo capsules to determine the effect on the rate of disease progression (Hoffman et al., 2004). The full-field cone electroretinogram (ERG) function was the primary outcome measure and recorded at baseline and at subsequent annual visits, where the total study duration is 4 years. The group difference in $\log _{t 0}$ cone ERG response amplitude increased linearly over time. Although the data analysis method used by the authors of this example suggested that a compound symmetric covariance structure was assumed, no information was given to check this assumption (Hoffman et al, 2004). Let us assume that a compound symmetric (CS) covariance structure is a plausible structure for the $\log _{10}$ cone ERG response amplitudes. The optimal allocation of the time-points is then at the end of the study, except for the first one or two time-points, depending on the correlation between two repeated measures (see Table 5.2). The relative efficiency of equidistant versus optimally allocated time-points would be about 0.56 (see Table 5.3). Thus, if the optimal allocation of time-points were used instead of equidistant time-points, the variance of the GLS treatment effect estimator might decrease by $44 \%$.

Table 5.6 Relative efficiency of used versus optimal designs for the body weight data (Diggle, 1990) and the ERG data (Hoffman et al., 2004), where the cost ratio $R=C_{k} / C_{2}=10$ and the budget is fixed.

\begin{tabular}{|c|c|c|}
\hline & body weight & ERG \\
\hline Covariance structure & ARI; $\rho_{1 K}=0.34$ & $\mathrm{CS} ; \rho_{1 K}=0.50^{(\mathrm{l})}$ \\
\hline \multirow[t]{2}{*}{ Used design } & $N=20^{(2)} ; K=5$ & $N=44 ; K=5$ \\
\hline & $t=0,11,2,3,4$ weeks & $t=0,1,2,3,4$ years \\
\hline \multirow[t]{2}{*}{ Optimal design } & $N=25 ; K=2$ & $N=44 ; K=5$ \\
\hline & $t=0,4$ weeks & $t=0,0,4,4,4$ years \\
\hline Relative efficiency & 0.8094 & 0.5556 \\
\hline
\end{tabular}




\section{Optimal time-points}

In Table 5.6, the results of the examples are summarized. Tablle 5.6 shows that the efficiency of a design can be substantially increased by choosing an optimal design.

\subsection{Discussion}

In the present paper, the optimal allocation of time-points, that is the allocation yielding the smallest variance of the generalized least squares (GLS) treatment effect estimator, was presented for $K=3,4$ and 5 repeated measures, different covariance structures and linearly divergent treatment effects. The relative efficiency of equidistant versus optimally allocated time-points and the relative efficiency of 2 versus $K=3,4$ and 5 were also presented.

The results showed that the optimal allocation and the rellative efficiencies depend strongly on the covariance structure. For a compound symmetric (CS) covariance structure, neither two nor more than two equally spaced measures yielded highly efficient treatment effect estimators. The optimal time-points for the additional measures were almost always at the end of the study. For a first-order auto-regressive (AR1) covariance structure, however, highly efficient treatment effect estimators were obtained for two repeated measures (one at the start and one at the end of the study). For a first-order auto-regressive covariance structure with measurement error ( $\mathrm{AR} 1+\mathrm{ME}$ ), the benefit in terms of efficiency of adding optimally spaced measures depends on the covariance parameter values. This benefit increases with increasing amount of measurement error and/or increasing auto-correlation, because the covariance structure then approaches compound symmetry. Adding a random intercept to the model hardly affected these results.

In conclusion, if the covariance structure resembles compound symmetry (the correlation between repeated measures decreases relatively slow with increasing time-lag), each patient should be measured more than twice and, except for the first measure, all measures should be taken at the end of the study. On the other hand, if the covariance structure resembles first-order auto-regression (the correlation between repeated measures decreases relatively fast with increasing time-lag), two repeated measures, i.e. one at the start and one at the end of the study, are sufficient to obtain highly efficient treatment effect estimators. The money saved by skipping additional measures can be spent on sampling more patients to gain efficiency. So the commonly used design with more than two equally spaced measures is neither highly efficient for a CS structure nor for an AR1 structure. 
Although prewious or pilot studies can be used to estimate the covariance structure of the data, misspecification of this structure may lead to an inefficient design. The results in Table 5.5 showed that when the covariance structure is unknown and the number of repeated measures per patient is smaller than five, choosing the optimal design based on compound symmetry seems to be the best choice in terms of robustness against misspecification. Choosing the optimal design based on a first-order auto-regressive structure may lead to a highly inefficient design if this structure does not hold (see Table 5.5). In most repeated measures studies, however, it is most likely that measures closer together in time will be more highly correlated than those separated in time (e.g. Everitt, 1995). So, in practice, choosing a design based on an AR1+ME structure instead of on a CS structure may be often a better choice in terms of robustness against misspecification of the covariance structure.

The covariance parameters $\left(\sigma_{0}^{2}, \sigma_{\varepsilon}{ }^{2}, \sigma_{s}{ }^{2}\right.$ and $\left.\rho_{\varepsilon}\right)$ are generally unknown and have to be estimated from the data. For their estimation, another allocation of time-points may be better than the present designs which are optimal for estimating the treatment effect parameter $\beta$. We are, however, primarily interested in the latter parameter. Note that, at least asymptotically, maximum likelihood estimators of fixed and covariance parameter are orthogonal (e.g. Searle, Casella \& McCulloch, 1992).

In the present paper, we assumed that the covariance structure is stationary and homogeneous across groups. Of course, subjects may be affected differently by the treatments. This implies that the variance of $y_{i j}$ changes over time. Thus, a natural extension to our work is to include non-stationary structures that may be heterogeneous across groups.

Further, we assumed that all planned measures were observed and that the treatment effect increased linearly over time. In practice, some repeated measures may be missing and/or the treatment effect may not increase linearly over time. In the first case, more repeated measures per patient and/or more patients are required to obtain efficient treatment effect. astimators. In the second case, more than two repeated measures are required to efficiently estimate the trend of the treatment effect. 


\section{References}

Arndt, S., Jorge, R., Turvey, C., \& Robinson, R. G. (2000). Adding subjects or adding measurements: which increases the precision of longitudinal research ?, Journal of Psychiatric Research, 34, 449-455.

Atkinson, A. C., \& Donev, A. N. (1996). Optimum Experimental Designs. Oxford: Clarendon Press.

Bloch, D. A. (1986). Sample size requirements and the cost of a randomized clinical trial with repeated measurements, Statistics in Medicine, 5, 663-667.

Diggle, P. J. (1990). Time Series: A Biostatistical Introduction. Oxford: Clarendon Press.

Diggle, P. J., Liang, K-Y, \& Zeger, S. L. (1996). Analysis of Longitudinal Data Oxford: Clarendon Press.

Everitt, B. S. (1995). The analysis of repeated measures: a practical review with examples, The Statistician, 44, 113-135.

Feighner, J. P. (1980). Trazodone, a triazolopyridine derivative, in primary depressive disorder, Joumal of Clinical Psychiatry, 41, 250-255

Frison, L. J., \& Pocock, S. J. (1997): Linearly divergent treatment effects in clinical trials with repeated measures: efficient analysis using summary statistics, Statistics in Medicine, $16,2855-2872$.

Furberg, C. D., Adams, H. P., Applegate, W. B., et al. (1994). Corony heart disease /myocardial infarction: Effect of Lovastatin on early carotid atherosclerosis and cardiovascular events, Circulation, 90, 1679-1687.

Gruttola, V., Lange, N., \& Dafni, U. (1991). Modeling the progression of HIV infection, Journal of the American Statistical Association, 86, 569-577.

Hoffman, D. R., Locke, K. G., Wheaton, D. H., Fish, G. E., Spencer, R, \& Birch, D. G. (2004). A randomized, placebo-controlled clinical trial of docosahexsenoic acid supplementation for X-linked retinitis pigmentosa, American.Journal of Ophthalmology, 137, 704-718.

Jennich, R. I. \& Schluchter, M. D. (1986). Unbalanced repeated-measures models with structured covariance matrices, Biometrics, 42, 805-820.

Jung, S-H, \& Ahn, C. (2003). Sample size estimation for GEE method for comparing slopes in repeated measurements data, Statistics in Medicine, 22, 1305-1315.

Liu, X. (2003). Statistical power and optimum sample allocation ratio for treatment and control having unequal costs per unit of randomization, Journal of Educational and 


\section{Chapter 5}

Behovioral Statistics, 28, 231-248.

Lui, K-J, \& Cumberland, W.G. (1992). Sample size requirement for repeated measurements in continuous data, Statistics in Medicine, 11, 633-641.

Maxwell, S. E (1998). Longitudinal designs in randomized group comparisons: when will intermediate observations increase statistical power?, Psychological Methads, 3, 275290 .

Ouwens, M. J. N. M., Tan, F. E. S. \& Berger, M. P. F. (2002). Maximin D-optimal designs for longitudinal mixed effects models, Biontetrics, 58, 735-741.

Overall, J. E., \& Doyle, S. R. (1994). Estimating sample sizes for repeated measurement designs, Controlled Clinical Trials, 15, 100-123.

Raudenbush, S. W. \& Chan, W. (1993) Application of hierarchical linear model to the study appendix of adolescent deviance in an overlapping cohort design. Journal of Clinical and Consulting Psychology, 61, 941-951.

Raudenbusli, S. W. \& Liu, X-F. (2001). Effects of study duration, frequency of observation, and sample size on power in studies of group differences in polynomial change, Psychological Methods, 6, 387-401.

Sehlesselman, J. J. (1973). Planning a longitudinal study: II. Frequency of measurement and study duration, Joumal of Chronic Diseases, 26, 561-570.

Schouten, H. J. A. (1999). Planning group sizes in clinical trials with a continuous outcome and repeated measures, Statistics in Medicine, 18, 255-264.

Searle, S. R., Casella, G., \& McCulloch, C. E. (1992). Variance Components. New York: John Wiley \& Sons.

$\operatorname{Tan}_{4}$ F. E. S. \& Berger, M. P. F (1999). Optimal allocation of time points for the random effects model, Communications in Statistics, Simnlations and Compulations, 28, 517 540.

Vickers, A. I. (2003). How many repeated measures in repeated measures designs? Statistical issues for comparative trials, BMC Medical Research Methodology, 3, 22 .

$\mathrm{Yi}_{\mathrm{i}}$ Q., \& Panzarella, T. (2002). Estimating sample size for tests on trends across repeated measurements with missing data based on the interaction term in a mixed model, Controlled Clinical Trials, 23, 481-496. 


\section{Appendix}

The optimal allocation of time-points for a compound symmetric covariance structare with $K=3$ repeated measures.

The formula for the variance of the GLS treatment effect estimator for a compound symmetric covariance structure can be written as

$$
\operatorname{Var}\left(\hat{\beta}^{\mathrm{oLs}}\right)=\frac{N_{C}+N_{T}}{N_{C} N_{T}} \times \frac{\sigma^{2}(1-\rho)\{1+(K-1) \rho\}}{\left.((K-2) \rho+1)\left(\sum_{i=2}^{K-1} t_{i}^{2}\right)-2 \rho\left(\sum_{i=2}^{K+1} t_{i}+\sum_{\substack{i, j=2 \\ i \neq j}}^{K+1} t_{i} t_{j}\right)+(K-2) \rho+1\right\}},
$$

where $\sigma^{2}=\left(\sigma_{0}{ }^{2}+\sigma_{e}{ }^{2}\right)$ is the variance of $y_{i,} \rho=\sigma_{0}{ }^{2} /\left(\sigma_{0}{ }^{2}+\sigma_{e}{ }^{2}\right)$ is the correlation between two repeated measures and $K$ is the number of repeated measures.

For $K=3$, the variance of the treatment effect estimator reduces to

$$
\operatorname{Var}\left(\hat{\beta}^{\text {ats }}\right)=\frac{N_{C}+N_{x}}{N_{C} N_{T}} \times \frac{\sigma^{2}(1-\rho)\{1+2 \rho\}}{(1+\rho) t_{2}^{2}-2 \rho t_{2}+(1+\rho)}
$$

where $0 \leq t_{2} \leq 1$. The denominator $(1+\rho) t_{2}^{2}-2 \rho t_{2}+(1+\rho)$ is minimal for $t_{2}=\rho /(1+\rho) \leq 1 / 2$ and maximal for $t_{2}=1$. Thus, the smallest variance of the GLLS treatment effect estimator is obtained for $t_{2}=1$. 
Chaprer 5 


\section{Optimal designs for clinical trials with second-order} polynomial treatment effects

This chapter has been submitted for publication with co-authors H. J. A. Schouten, G. J. P. van Breukelen and M. P. F. Berger 


\title{
Chapter 6
}

\begin{abstract}
The effect of adding intermediate measures on the efficiency of treatment effect estimation is considered for a second-order polynomial treatment effect, equidistant timepoints, different covariance structures and two optimality criteria, assuming either a fixed number of subjects or a fixed study budget. The benefit of adding intermediate measures (at the expense of subjects) depends strongly on the assumed covariance structure and is hardly affected by the two optimality criteria that were used $\left(D_{s}\right.$ or $\left.c\right)$. For a fixed number of subjects, the increase in efficiency by adding intermediate measures is large for a compound symmetric structure and small for a first-order auto-regressive structure. For a first-order autoregressive structure with measurement error, the results depend on the covariance parameter values. For a fixed budget and a linear cost function, the design with only three measures per subject is often highly efficient. If the stnucture resembles compound symmetry and the cost per subject is eight or more times larger than the cost per repeated measure, however, more than three measures are required to obtain highly efficient treatment effect estimators. If the covariance structure is unknown, the optimal design based on a first-order autoregressive structure with measurement error is preferable in terms of robustness against misspecification of the covariance structure. Given a design with 3 repeated measures and a second-order pollynomial treatment effect, equidistant time-points are either optimal $\left(D_{s^{-}}\right)$or highly efficient (c-optimality criterion).
\end{abstract}

Keywords: Auto-correlation; Compound Symmetry; c- and $\mathrm{D}_{\mathrm{s}}$-optimality criteria; Optimal design; Repeated Measures. 


\subsection{Introduction}

In planning repeated measures studies, decisions about the number of subjects and number of repeated measures per subject depend on the pattern of treatment effects and the covariance structure of the repeated measures (e.g. Overall \& Doyle, 1994). An overview of the literature with guidelines on sample size and power calculations for repeated measures designs can be found in Raudenbush and Liu (2001). Although a linear growth or linear change function is assumed in many repeated measures studies, this pattern may not be strictly linear (e.g. Overall and Doyle, 1994). For example, the difference between two groups may at first increase relatively fast and then stabilize or even decrease over time (see Figure 6.1). For non-linear polynomial treatment effects with two or more parameters, the interest is often focussed on the highest-order polynomial treatment effect parameter (e g. Raudenbush \& Liu, 2001).
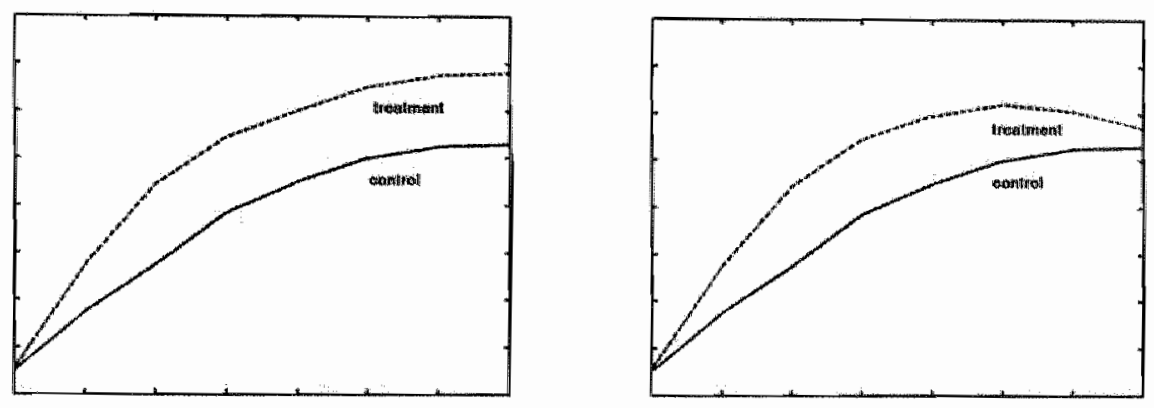

Figure 6.1. Two examples of second-order polynomial treatment effect over time.

In the present paper, second-order polynomial treatment effects, i.e. $\beta_{1} \gamma+\beta_{2} t^{2}$, will be considered, where no group effect is present at the start of the study ( $t=0$ ), and interest is in estimating both the linear $\beta_{1}$ and the quadratic $\beta_{2}$ treatment effect parameters as efficiently as possible. Two questions are dealt with. The first question is whether only three equally spaced measures are sufficient to efficiently estimate the treatment effect parameters or whether intermediate measures need to be added to obtain this goal? The second question concerns the optimal allocation of the time-points for a three repeated measures design, i. e do equidistant time-points yield highly efficient treatment effect estimators or should the measures be nonequidistant? 
A linear cost function will be specified to account for the costs of sampling and measuring subjects (e.g. Bloch, 1986; Lui Cumberland, 1992). Optimal designs will be computed for a specified linear cost function, a fixed study budget and different covariance structures. Two different optimality criteria will be used. The $D_{s}$-optimality criterion, because it has nice properties (see Atkinson \& Donev, 1996, p. 106), and the difference between the area under the curves $(A U C)$ of the treatment and control groups, because it is often used in repeated measures studies with a second-order polynomial treatment effect (e.g. Matthews et al., 1990; Pham et al., 1999; Schiff, 2003). The effect of adding intermediate, equally spaced measures on the $D_{s}$ and $A U C$-efficiencies are also computed assuming a fixed number of subjects instead of a fixed budget for sampling and measuring subjects.

In Section 6.2, the mixed effects model is introduced and different covariance structures and optimality criteria are discussed. For a fixed number of subjects, the effect of adding intermediate, equally spaced measures is discussed in Section 6.3. In Section 6.4, the $D_{s^{-}}$and $A U C$-optimal combination of group sizes and number of repeated measures per subject is calcullated for different covariance structures, equidistant time-points, a linear cost function and a fixed study budget. Misspecification of the covariance structure is discussed in Section 6.5. In Section 6.6, the optimal allocation of time-points is considered for the design with three repeated measures per subject. The numerical results are illustrated by an example in Section 6.7. Finally, the conclusions are summarized and discussed in Section 6.8 .

\subsection{Mixed effects model and optimal design criteria}

\subsubsection{Model}

For subject $i$, let $y_{i j}$ be the $j$ th repeated measurement of a continuous outcome variable measured at time $t_{j}(i=1,2, \ldots, N ; j=1,2, \ldots, K)$. For simplicity, two groups are considered, i.e. each subject is randomized either to the (active) treatment group or to the placebo or control group. Further, the first measure is taken at $t_{1}=0$, i.e. just before randomization, and the fixed total duration of the treatment period is rescaled to unity $\left(t_{K}=1\right)$. In the present paper, the following mixed effects model is used,

$$
y_{i}=X_{j} \beta+Z b_{i}+c_{i}+e_{i_{3}}
$$

where 


$$
\boldsymbol{X}_{i} \boldsymbol{\beta}=\left(\begin{array}{cccccc}
1 & 0 & \cdots & 0 & G_{i} \times t_{1} & G_{i} \times i_{1}^{2} \\
0 & \ddots & \ddots & \vdots & \vdots & \vdots \\
\vdots & \ddots & \ddots & 0 & \vdots & \vdots \\
0 & \cdots & 0 & 1 & G_{i} \times t_{K} & G_{i} \times i_{K}^{2}
\end{array}\right)\left(\begin{array}{c}
\mu_{1} \\
\vdots \\
\mu_{k} \\
\beta_{1} \\
\beta_{2}
\end{array}\right) \text { and } Z b_{i}=\left(\begin{array}{l}
1 \\
\vdots \\
1
\end{array}\right)\left(b_{0 i}\right)
$$

Here $\beta_{1}$ and $\beta_{2}$ are the linear and quadratic treatment effect parameters, respectively, and $G_{i}$ is an indicator of treatment; $G_{i}=1$ if subject $i$ is in the treatment group and $G_{i}=0$ otherwise. Thus, $y_{i j}$ has expectation $\mu_{j}$ in the control group and $\mu_{j}+\beta_{3} t_{j}+\beta_{2} t_{j}^{2}$ in the treatment group, i.e. no group effect is assumed at time $h_{1}=0$, due to randomization. Further, the random effects vector $b_{i}$, the component of auto-correlation $\varepsilon_{i}$ and the additional component of measurement error $e_{i}$ are assumed to be independently, normally distributed with mean vectors $\theta$ and covariance matrices $D=\left(\sigma_{0}^{2}\right), \sigma_{E}^{2} \boldsymbol{H}$ and $\sigma_{e}{ }^{2} I_{K}$, respectively. Here $\boldsymbol{I}_{K}$ is the $K$-dimensional identity matrix and $\boldsymbol{H}$ has a first-order auto-regressive (AR1) correlation structure, i.e. the correlation $\rho_{\varepsilon}{ }^{\tau}$ between $\varepsilon_{i j}$ and $\varepsilon_{i j}$ decreases with increasing time-lag $\tau=\left|t_{j}-t_{j}\right|$ and $\rho_{\varepsilon}$ is the correlation between $\varepsilon_{i 1}$ and $\varepsilon_{i K}$. Thus, the covariance matrix of the outcome vector $y_{i}$ is $\operatorname{Var}\left(y_{i}\right)$ $=V=Z D Z^{\prime}+\sigma_{\tilde{*}}^{2} \boldsymbol{H}+\sigma_{e}{ }^{2} \boldsymbol{I}_{K}$, which is homogeneous across groups and subjects.

With these three sources of random variation (a random intercept, auto-correlation and measurement error), quite general stationary covariance structures may be obtained (see e.g. Diggle, Liang and Zeger, 1996, p.89). Random slopes may be added to this model to obtain non-stationary covariance structures, but only stationary structures are considered in this paper to bring our key issues into focus.

\subsubsection{Covarionce structures}

Five well-known covariance structures can be obtained from model (6.1): first-order auto-regression (AR1), first-order auto-regression with measurement error (AR1+ME), random intercept with measurement error (RI+ME) or serial correlation (RI+ARI) or both $(\mathrm{RI}+\mathrm{AR} 1+\mathrm{ME})$. In Table 6.1, the dependence of the correlation $\rho_{j j^{\prime}}$ between $y_{i j}$ and $y_{i j^{\prime}}$ on the time-lag $\tau=\left|t_{j}-t_{j}\right|$ is shown for these covariance structures. Notice that a RT+ME structure is equivalent to the compound symmetric (CS) structure. 
Table 6.1 Correlation $p_{j j^{\prime}}$ between $y_{i j}$ and $y_{i j^{\prime \prime}}$ for covariance structures based on a random intercept $(\mathrm{RI})$, auto-correlation (AR 1$)$ and measurement error (ME).

Correlation $p_{i j^{\prime}}$ between $y_{i j}$ and $y_{i j^{\prime}}$

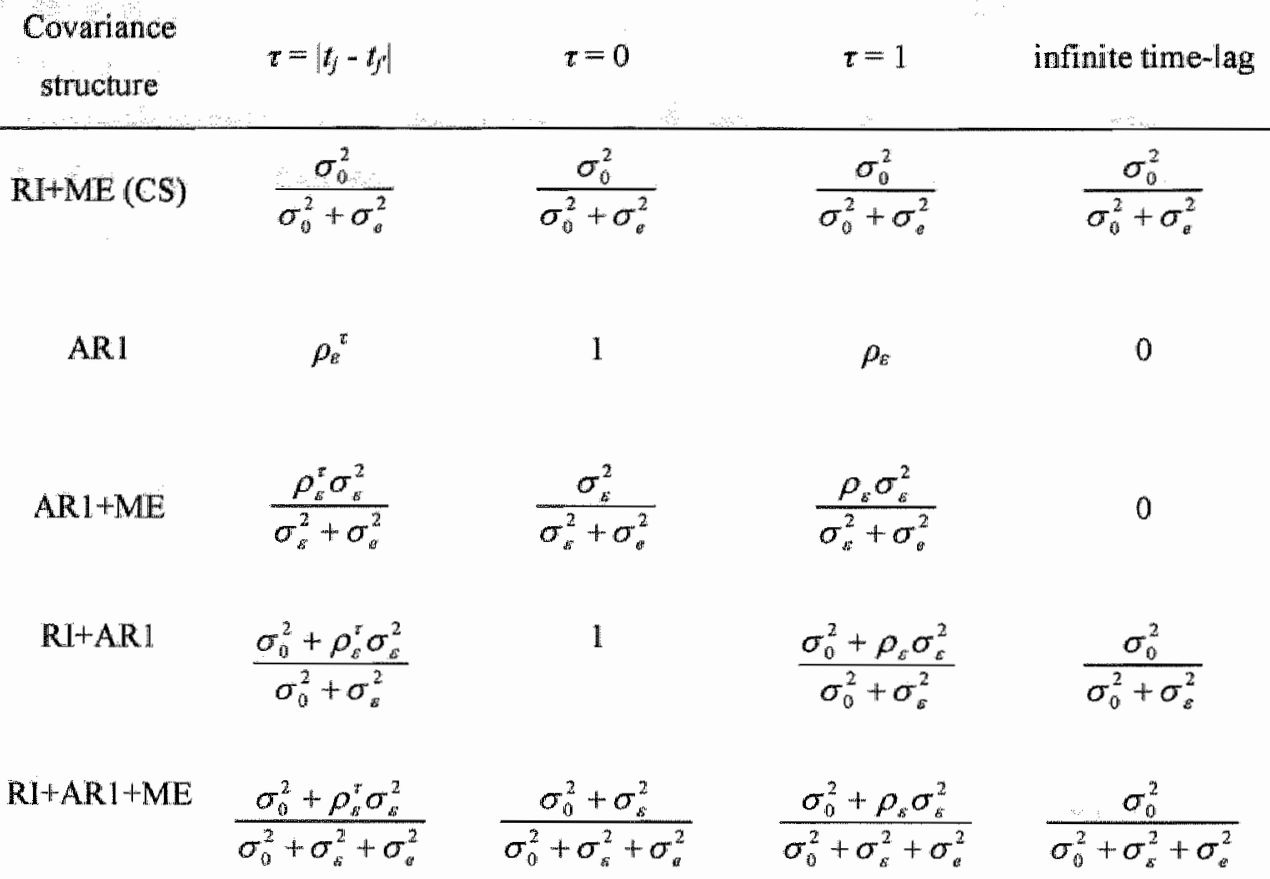

Table 6.1 shows that except for a CS $(=\mathrm{RI}+\mathrm{ME})$ structure, the correlation $\rho_{j j^{\prime}}$ between $y_{i j}$ and $y_{i^{\prime}}$ decreases with increasing time-lag $\tau$. For an AR1 structure, the correlation $\rho_{j j^{\prime}}$ decreases from 1, obtained when $t=0$, to 0 , obtained for an infinite time-lag. Table 6.1 also shows that measurement error ensures a correlation $p_{y j}$ smaller than 1 and a random intercept leads to a correlation $\rho_{j l}$ larger than 0 . If the measurement error variance $\sigma_{e}{ }^{2}$, the correlation $\rho_{\varepsilon}$ between $\epsilon_{k 1}$ and $\varepsilon_{i, k}$ and/or the random intercept variance $\sigma_{0}{ }^{2}$ (for a RI+ARI + ME structure) increase, the $\mathrm{ARI}+\mathrm{ME}$ and $\mathrm{RI}+\mathrm{ARI}+\mathrm{ME}$ structures approach a $\mathrm{CS}$ structure, because the difference between the correlation $\rho_{1} K$ between first and last measures and the largest correlation, which is obtained when time-lag $\tau=0$, then decreases. Without loss of generality the variance of $y_{i j}$ will be fixed at 1 for all covariance structures. 


\subsubsection{Optimality criteria}

For the mixed effects model (6.1) the generalized least squares (GLS) estimator of $\beta$ and its variance are

$$
\hat{\beta}^{\mathrm{GLS}}=\left(\sum_{i=1}^{N} \boldsymbol{X}_{i}^{\prime} \boldsymbol{V}^{-1 \mathrm{~K}} \boldsymbol{X}_{i}\right)^{-11} \sum_{i=1}^{N} \boldsymbol{X}_{i}^{\prime} \boldsymbol{V}^{-1} \boldsymbol{y}_{i}
$$

and

$$
\operatorname{Var}\left(\hat{\boldsymbol{\beta}}^{\mathrm{acs}}\right)=\left(\sum_{i=1}^{N} \boldsymbol{X}_{i}^{\prime} \boldsymbol{V}^{-1} \boldsymbol{X}_{i}\right)^{-\|}
$$

where $V=Z D Z^{n}+\sigma_{\varepsilon}^{2} \boldsymbol{H}+\sigma_{e}^{2} \boldsymbol{I}_{K}$ (e.g. Diggle, Liang and Zeger, 1996, p. 59).

A commonly used optimality criterion is the D-optimality criterion, i.e. the determinant of the covariance matrix (6.4) of $\hat{\boldsymbol{\beta}}^{\text {oLs }}$ (see e.g. Atkinson \& Donev, 1996, p. 42). Although other optimality criteria are available, like the $A$ - and E-criteria, the D-optimality criterion is preferred, because "D-optimal designs for quantitative variables do not depend upon the scale of the variables contained in the design matrices $X_{\hat{i}}{ }^{\prime}$ and minimize the area of the simultaneous confidence region of the fixed parameters (see Atkinson and Donev, 1996, p. 106-107). In the present paper, we are only interested in the treatment effect parameters $\beta_{1}$ and $\beta_{2}$. Therefore, the determinant of the covariance matrix of the GLS treatment effect estimators $\hat{\beta}_{1}^{\mathrm{GL} s}$ and $\hat{\beta}_{z}^{\mathrm{GLS}}$ is minimized. This optimality criterion is called a $D_{s}$-optimality criterion, because interest is only in a subset of $s$ parameters (see Atkinson \& Donev ${ }_{1096, p}$. 109). In the present paper, we also used a c-optimality criterion; a c-optimal design minimizes the variance of a linear combination of the parameter estimators $c^{\prime} \hat{\beta}^{\text {ols }}$. Here we have chosen $c^{\prime}=(0, \ldots, 0,1 / 2,1 / 3)$, i.e. $c \hat{\beta}^{\text {GLs }}=1 / 2 \hat{\beta}_{1}^{\text {GLs }}+1 / 3 \hat{\beta}_{2}^{\text {GLs }}$, because if the curves of the treatment and control groups do not intersect for $0<t<1$, this corresponds to the difference between the areas under these curves $(A U C)$. This can be verified by integrating the treatment effect $\beta_{1}+$ $\beta_{2} t^{2}$ over the time interval $[0,1]$. This criterion is often used in repeated measures studies with second-order polynomial treatment effects (e.g. Matthews et al., 1990; Schiff, 2003). If the curves intersect, the difference between the areas under the curves $(A C C)$ is clinically less 


\section{Chapter 6}

useful and becomes a complicated function of $\hat{\beta}_{1}^{\text {ans }}$ and $\hat{\beta}_{2}^{\mathrm{GLS}}$, but this is beyond the scope of the present paper. Notice that the $D_{k}$-optimality criterion can be used if the curves intersect.

Two designs can be compared by the relative efficiency eff( $\left.D_{1} \mid D_{2}\right)$ of design $D_{1}$ versus design $D_{2}$. For the $D_{5}$ and $A U C$-optimality criterion, this relative efficiency eff $\left(D_{1} \mid D_{2}\right)$ is

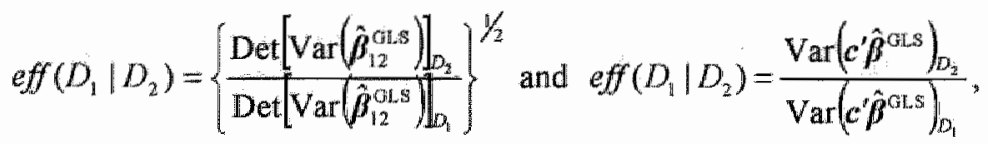

respectively, where $\hat{\beta}_{12}^{\text {GLS }}=\left(\hat{\beta}_{1}^{\mathrm{CLS}}, \hat{\beta}_{2}^{\mathrm{CLLS}}\right)^{\prime}$ and $c^{\prime \prime}=(0, \ldots, 0,1 / 2,1 / 3)$. This relative efficiency can be greater or less than 1 , depending on which design, $D_{1}$ or $D_{2}$, goes with the smaller variance $\operatorname{Var}\left(c^{*} \hat{\beta}^{\text {aLs }}\right)$ (AUC-optimality criterion) or smaller determinant ( $D_{s}$-optimality criterion). For example, if eff( $D_{1}\left(D_{2}\right)=0.8$, design $D_{2}$ is more efficient than design $D_{\mathrm{l}}$. This latter design would then require $[(1-0.8) / 0.8] \times 100 \%=25 \%$ additional subjects to obtain the same variance $\left(A \cup C\right.$-optimality criterion) or determinant $\left(D_{s}\right.$-optimality criterion) as design $D_{2}$.

\subsection{Effect of adding intermediate measures for a fixed sample size}

In this section, the effect of adding intermediate measures on the $D_{3^{-}}$and $A U C$ efficiency of the design with three equally spaced measures $(t=0,0.5,1)$ is computed with equations (6.4) and (6.5) for a fixed number of subjects, equidistant time-points and different covariance structures. In Section 6.4, a fixed total budget for sampling and measuring subjects will be assumed (instead of a fixed number of subjects), which means that adding repeated measures per subject will be at the expense of subjects. Although the covariance structure is unknown in the design stage of a study, we assume that the covariance structure is known in both sections. The effect of misspecification of the covariance structure will be discussed in Section 6.5 .

For a fixed number of subjects, the relative efficiency eff $\left(D_{\|} \mid D_{2}\right)$ of the design $D_{1}$ with 3 equally spaced measures versus the design $D_{2}$ with $K$ equally spaced measures is plotted as function of the number of time-points $K(=3, \ldots, 25)$ for $D_{s^{-}}$(Figure 62a) and $A U C$ optimality criteria (Figure 6.2b). Here different curves are plotted for the CS, ARI and AR1-ME covariance structures, where the correlation $\rho_{\mathrm{IR}}$ between first and last measures is $0.3,0.5$ and 0.7 . The curves for the RI+ARI and RI+ARI+ME structures were omitted, 
because they were similar to those for the ARI and ARI+ME structures, respectively, i.e. the results were hardly affected by adding a random intercept to an ARI or AR1+ME structure. Variance and covariance parameters are considered that are not too extreme to clearly distinguish the specific covariance structures. For example, if $p_{\mathrm{K} K}$ is very small, $\mathrm{AR} 1$ and $\mathrm{CS}$ structures both approximate an independence structure. Although the relative efficiency is plotted for the AR $1+\mathrm{ME}$ structure with $\sigma_{\varepsilon}{ }^{2}=0.8$ and $\sigma_{e}{ }^{2}=0.2\left(\rho_{j y^{\prime}} \leq 0.8\right.$ for all $j$ and $\left.j\right)$, the results were similar for $0.4 \leq \sigma_{\varepsilon}{ }^{2} \leq 0.9\left(0.6 \geq \sigma_{e}{ }^{2} \geq 0.1\right)$. If $\sigma_{\varepsilon}{ }^{2}<0.4$ or $\sigma_{\varepsilon}{ }^{2}>0.9$, however, the $\mathrm{AR} 1+\mathrm{ME}$ structure is close to a $\mathrm{CS}$ or an $\mathrm{AR} 1$ structure, respectively (remember $\operatorname{Var}\left(y_{i j}\right)=1$ ).

(a)

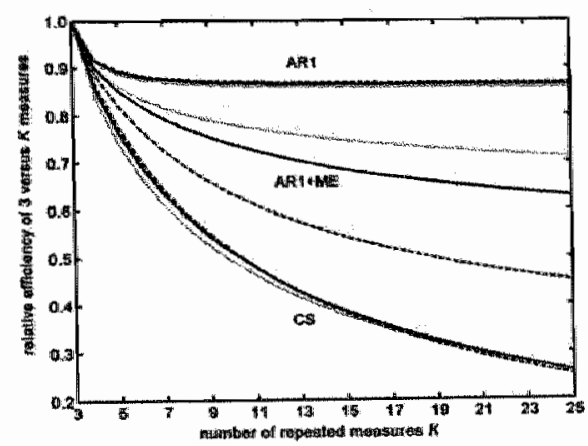

(b)

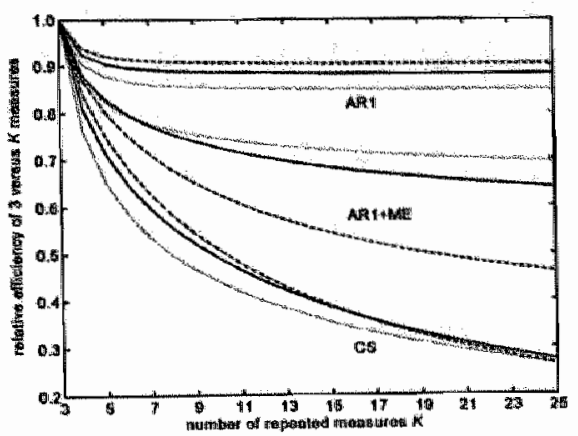

Figure 6.2 Rellative efficiency of 3 versus $K$ repeated measures for a fixed sample size and $\mathrm{CS}, \mathrm{AR} 1$ and $\mathrm{AR} 1+\mathrm{ME}\left(\sigma_{\varepsilon}{ }^{2}=0.8 ; \sigma_{\varepsilon}{ }^{2}=0.2\right)$ structures: a) $D_{s}$-optimality criterion, b) $A U C$ optimality criterion. Dotted line: $\rho_{1 K}=0.3$, solid line: $\rho_{1 K}=0.5$ and dashed line: $\rho_{1 K}=0.7$.

Figure 6.2 shows that the plots of the relative efficiency of 3 versus $K$ equally spaced measures are similar for $D_{s}$ (Figure 6.2a) and $A U C$-optimality criteria (Figure 6.2b). Although the relative efficiency of 3 versus $K$ equally spaced measures decreases with increasing $K$, the amount of decrease depends strongly on the covariance structure (see Figure 6.2). For a CS structure, the decrease is large, whereas it is small for AR1. For an AR1+ME structure, the benefit of adding intermediate measures increases 1) with increasing autocorrelation $\rho_{\varepsilon}$ or 2 ) with increasing measurement error variance $\sigma_{e}{ }^{2}$ (not shown here). This can be explained as follows. If $\rho_{\varepsilon}$ and/or $\sigma_{e}{ }^{2}$ increase, the difference between the largest correlation (obtained when the time-lag $\tau=0$ ) and the correlation $\rho_{K}$ between first and last 


\section{Chapter 6}

measures decreases (see Table 6.1). As a result; the ARI + ME structure approaches the $\mathrm{CS}$ structure and the benefit of adding intermediate measures increases.

Note that for ARI and CS structures, the benefit of adding intermediate measures is hardly affected by increasing $\rho_{1 K}$ from 0.3 to 0.5 or 0.7 . The results would change, however, if $\rho_{\mathrm{U} K}$ approaches 0 or 1 . For ARI structure with $\rho_{1 K}<0.01$, for example, the benefit of adding intermediate measures becomes large, because then the AR1 structure resembles the independence structure.

In conclusion, for a fixed number of subjects, fixed study duration and a CS structure, the efficiency of a design increases fast with increasing number of repeated measures. For ARI and RI+AR1 structures, howewer, the increase in efficiency by adding intermediate, equally spaced measures is very slow. For $A R 1+M E$ and $R I+A R 1+M E$ structures, the benefit of adding intermediate measures increases with increasing auto-correlation $\rho_{\varepsilon}$ and/or increasing amount of measurement error, because then the $A R 1+M E$ and $R I+A R 1+M E$ structures approach a CS structure.

In short, the benefit of adding intermediate measures is large if the difference between the correlation $\rho_{I K}$ between first and last measures and the correlation $\rho_{j, j+1}$ between successive measures is small. This difference is smallest for CS and largest for AR1.

\subsection{Optimal group sizes and number of measures for a fixed budget}

\subsubsection{Cost function}

In practice, budget constraints may often limit the number of subjects and the number of repeated measures. As a consequence, researchers have to deal with the question which combination of group sizes and number of repeated measures will be cheapest to obtain prespecified efficiencies of the treatment effect estimators. To deal with this question, a linear cost function is defined as

$$
C=C_{1} N+C_{2} N K
$$

where $C$ is the total budget for sampling and measuring subjects, excluding overhead cost, $C_{1}$ is the cost per subject, i.e cost for recruitment and cost of treatment, $C_{2}$ is the cost per repeated measure and $N=N_{T}+N_{C}$ is the total number of subjects. For simplicity, we assume that the cost per subject are equal for both treatment and control groups. The optimal fraction 
$f=N_{T} /\left(N_{T}+N_{C}\right)$ of subjects randomly assigned to the treatment group is then equal to $1 / 2$. We also assume that sampling a subject costs the same or more than obtaining a repeated measure, i.e. the cost-ratio $R=C_{1} / C_{2} \geq 1$. Equation (6.6) can be rewritten as $N=C /\left\{C_{2}(R+K)\right\}$.

Given this cost function, the optimal combination of sample size $N$ and number of repeated measures $K$ will be calculated by minimizing the $D_{s}$ - or $A U C$-optimality criterion within a certain maximal allowable budget. Note that this is equivalent to minimizing the total budget given a maximum allowable value of the $D_{s}$ - or $A U C$-criterion (e.g. Moerbeek, van Breukelen \& Berger, 2000).

Since the optimal combination of $N$ and $K$ depends on the relative costs of subjects and repeated measures, a large range for the cost ratio $R=C_{1} / C_{2}=1,10,100$ is considered. Further, for practical reasons, the number of repeated measures is bound to a maximum of 25 . Note that the total budget $C$ is irrelevant for the relative efficiency of 3 versus $K$ repeated measures, which depends on $K$ and the cost ratio $R$. The total budget $C$ is, however, relevant for the number of subjects $N$ and thereby for power, whether $K=3$ or not.

\section{4 .2 Reswits}

In Figures $6.3-6.5$, the relative efficiency of 3 versus $K$ repeated measures is plotted against $K(=3, \ldots, 25)$ for $\mathrm{CS}, \mathrm{AR} 1$ and AR1+ME structures, respectively, where a fixed budget is assumed and the $D_{s}$-optimality criterion is used. Separate lines are plotted for the correlation between first and last measures $\rho_{1 K}=0.3,0.5,0.7$ and cost ratio $R=C_{1} / C_{2}=1,10$, 100. If the curves of the treatment and control groups do not intersect for $0<t<1$, similar results are obtained for the $A U C$-optimality criterion. Since the $D_{s}$-optimality criterion can also be used if the curves intersect, only the plots for the $D_{s}$-optimality criterion are presented here. Although the relative efficiency is plotted for the AR $1+\mathrm{ME}$ structure with $\sigma_{s}{ }^{2}=0.8$ and $\sigma_{e}{ }^{2}=0.2\left(\rho_{j /} \leq 0.8\right.$ for all $j$ and $\left.j^{\prime}\right)$, the results were similar for $0.4 \leq \sigma_{r}{ }^{2} \leq 0.9\left(0.6 \geq \sigma_{i j}{ }^{2} \geq 0.1\right)$. For $\sigma_{\varepsilon}{ }^{2}<0.4$ or $\sigma_{\varepsilon}{ }^{2}>0.9$, the ARI+ME structure resembles a CS or an AR1 structure, respectively. Adding a random intercept to the model hardly changed the results, i.e. similar results were obtained for $A R 1$ and $R I+A R 1$ structures and likewise for $A R 1+M E$ and $\mathrm{RI}+\mathrm{AR} 1+\mathrm{ME}$ structures, because the effect of adding a random intercept is minimal for $\tau=0$ and maximal for an infinite time-lag. 


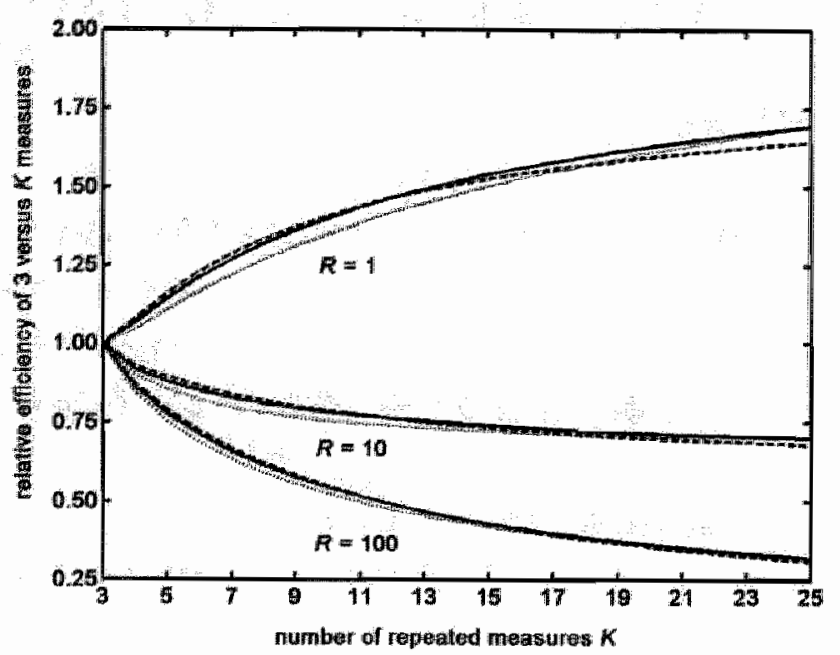

Figure 6.3 $D_{s}$-optimality criterion: relative efficiency of 3 versus $K$ repeated measures for a fixed budget and CS. Dotted line: $\rho_{1 K}=0.3$, solid line: $\rho_{1 K}=0.5$ and dashed line: $\rho_{1 K}=0.7$; $K_{\mathrm{opt}}=3$ for $R=C_{1} / C_{2}=1 ; K_{\text {opt }}=25$ for $R=10$ or $R=100$.

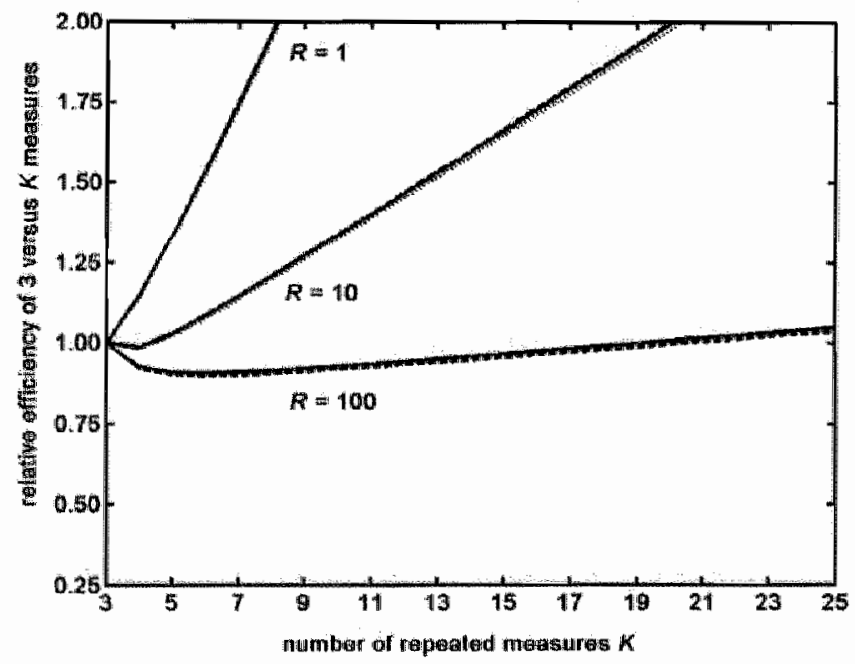

Figure 6.4 $D_{y}$-optimality criterion: relative efficiency of 3 versus $K$ repeated measures for a fixed budget and AR1. Dotted line: $\rho_{1 K}=0.3$, solid line: $\rho_{1 K}=0.5$ and dashed line: $\rho_{\mathrm{L} K}=0.7$, $K_{\text {opt }}=3$ for $R=C_{1} / C_{2}=1 ; K_{\text {opt }}=4$ for $R=10 ; K_{\text {opt }}=6$ for $R=100$. 


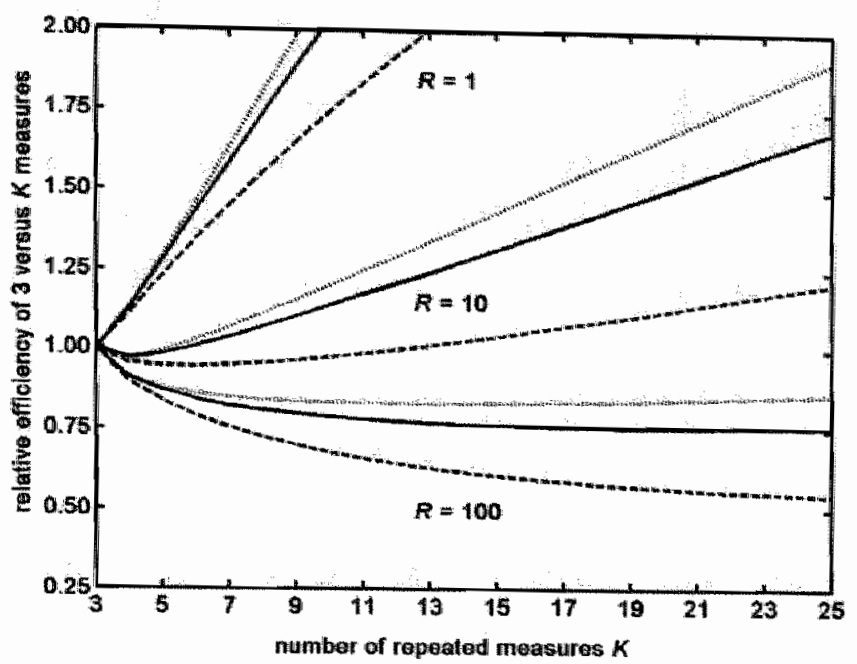

Figure $6.5 D_{s}$-optimality criterion: the relative efficiency of 3 versus $K$ repeated measures for a fixed budget and $A R 1+M E$ structure with $\sigma_{\varepsilon}^{2}=0.8$ and $\sigma_{e}^{2}=0.2$. Dotted line: $\rho_{\| K}=0.3$, solid line: $\rho_{1 K}=0.5$ and dashed line: $\rho_{1 K}=0.7 ; K_{\mathrm{spt}}=3$ for $R=C_{1} / C_{2}=1 ; K_{\mathrm{ept}}=4,4,6$ for $R$ $=10$ and $\rho_{1 K}=0.3,0.5,0.7$, respectively; $K_{\mathrm{opt}}=12,20,25$ for $R=100$ and $\rho_{i}=0.3,0.5,0.7$, respectively.

Figure 6.3 shows that for a cost ratio $R=10$ or 100 and a CS structure, adding intermediate, equally spaced measures to a design with three equally spaced measures at the expense of subjects is beneficial, whereas the three repeated measures design is optimal if $R=$ 1. More specifically, for cost ratio $R \leq 5$, the three repeated measures design is still highly efficient, i.e relative efficiency of 3 versus $K$ repeated measures is always larger than 0.9 , whereas for $R \geq 8$, the relative efficiency may be smaller than 0.8 (not shown here). For an AR1 structure, the three repeated measures design is either optimal or highly efficient, i.e. the relative efficiency is always larger than or equal to 0.9 (see Figure 6.4). Figure 6.4 also shows that for an $A R 1$ structure and a cost ratio $R=1$ or 10 , too many repeated measures can lead to a very inefficient design. Figure 6.5 shows that for an AR $1+M E$ structure and a cost ratio $R=$ $C_{1} / C_{2}=1$ or 10 , the design with three equally spaced measures is highly efficient. For $R=$ 100 , however, the increase in efficiency by adding intermediate, equally spaced measures to a design with three equally spaced measures nay be large, especially if the correlation between 


\section{Chapter 6}

first and last measures $\rho_{\| K^{\circ}}=0.7$, i.e. AR $1+\mathrm{ME}$ resembles CS, because $\rho_{j j^{\circ}} \leq 0.8$ for all $j$ and $j^{r}$ (since $\sigma_{E}{ }^{2}=0.8$ and $\sigma_{e}{ }^{2}=0.2$ ).

In conclusion; for a fixed budget, a design with three equally spaced measures is almost always highly efficient. If the covariance structure resembles compound symmetry (CS) and the cost per subject $C_{1}$ is eight or more times larger than the cost per repeated measures $C_{2}$, however, adding intermediate measures to the three repeated measures design can be beneficial.

\subsection{Misspecification of the covariance structure}

Previous sections show that the effect of adding intermediate measures (at the expense of subjects) relies heavily on the assumed covariance structure, which is often unknown at the design stage of a study. Although previous or pilot studies may be utilized to estimate the covariance structure, misspecification of the covariance structure may lead to an inefficient design.

In this section, the efficiency of a design based on an incorrect covariance structure will be compared with the one based on the correct covariance structure. Since the $D_{s}$ - and $A U C$-optimal designs are similar (see Figure 6.2), the effect of misspecification of the covariance structure will hardly be affected by the choice between these two optimality criteria. Therefore, the effect of misspecification is presented only for the $D_{s}$-optimality criterion. For a fair comparison between the efficiencies of the two designs, the linear cost function (6.6) will be used, in which the budget for sampling and measuring subjects will be assumed fixed. As an example, we will assume that a subject costs ten times as much as a repeated measure per subject, i.e. the cost ratio $R=C_{1} / C_{3}=10$, and that the correlation $p_{1 K}$ between first and last measures will be 0.5 . For practical reasons, the number of repeated measures per" subject $K$ will be bound to a maximum of 25 measures.

In Table 6.2 , the relative efficiency of a design based on the assumed (incorrect) versus correct covariance structure is presented for compound symmetry (CS), first-order auto-regression (AR1) and first-order auto-regression with measurement error (AR1+ME). For $\mathrm{AR} 1+\mathrm{ME}$ structure, we assumed that the auto-correlation variance $\sigma_{\varepsilon}{ }^{2}=0.6$ and the

measurement error variance $\sigma_{e}{ }^{2}=0.4\left(\rho_{i j} \leq 0.6\right.$ for all $j$ and $\left.j^{*}\right)$, because the AR $1+\mathrm{ME}$ structure with $\sigma_{6}{ }^{2}=0.8$ and $\sigma_{e}{ }^{2}=0.2$ led to the same optimal number of repeated measures as for an AR1 structure (see Figures 6.4 and 6.5). Adding a random intercept to an AR $\mathbb{1}$ or $A R 1+M E$ structure did not substantially affect the results. 
Table 6.2 Relative efficiency of a design based on assumed versus correct covariance structure; $D_{5}$-optimality criterion, correlation between first and last measures $\rho_{\mathrm{LK}}=0.5$, total budget $C$ is fixed, cost ratio $R=C_{1} / C_{2}=10$ and maximum number of repeated measures $K_{\text {numx }}$ $=25$.

\begin{tabular}{cccc}
\hline Assumed \Correct & CS & AR & AR1+ME \\
\hline CS & 1 & 0.4247 & 0.8713 \\
AR1 & 0.7622 & 1 & 0.9565 \\
AR1 1 ME $^{(1)}$ & 0.8647 & 0.8177 & 1 \\
\hline
\end{tabular}

${ }^{(1)}$ The measurement error variance $\sigma_{e}{ }^{2}=0.4$ and serial error variance $\sigma_{t}^{2}=0.6$.

Table 6.2 shows that misspecification of the covariance structure may lead to an inefficient design. In particular, if the optimal design were incorrectly based on a CS structure, while the correct covariance structure is AR1, the loss in efficiency by misspecification would be about $57 \%$. Choosing the optimal design based on an AR1 or AR1+ME structure with $\sigma_{e}{ }^{2}=0.4$ and $\sigma_{s}{ }^{2}=0.6$ seems to be a better choice in terms of robustness against misspecification of the covariance structure; the loss in efficiency is smaller than $24 \%$ or $19 \%$, respectively, at least for $R=10$ and $\rho_{1 K}=0.5$ (see Table 6.2).

\subsection{Optimal allocation of time-points for the three measures design}

Figures $6.3-6.5$ show that the design with three equally spaced measures is often highly efficient relative to the design with $K>3$ equally spaced measures. For the three repeated measures design, the optimal allocation of time-points $\left[t_{1}, t_{2 n} t_{3}\right]$ in design space $[0,1]$ will be computed for the $D_{s^{-}}$and $A U C$-optimality criteria. Notice that for three repeated measures, the optimal allocation of time-points is obtained by minimizing the $D_{5}-$ or $A U C^{-}$optimality criterion as a function of $t_{24}$ since the first and last time-points were fixed, $t_{t}=0$ and $t_{3}=1$. This optimal choice can be caiculated analytically for the CS structure and $D_{s}$ optimality criterion (see Appendix; Bischoff, 1995; Huang, Chang \& Wong, 1995). For the other structures and/or $A U C$-optimality criterion, the optimal choice was calculated numerically. In Table 6.3, the optimal allocation of time-points and the relative efficiency of equidistant versus optimally allocated time-points is presented for the $D_{s}$ - and $A U C$-optimality criteria, and CS, ARI and ARI+ME structures, where the correlation $\rho_{\mathrm{K}}$ between first and last measures is $0.3,0.5$ or 0.7 . 
Table 6.3 Optimal allocation of time-points $\left[0, t_{2}, 1\right]$ for a three repeated measures design and relative efficiency $e f f\left(D_{\text {equit }}\left(D_{\text {opt }}\right)\right.$ of equidistant $\left(D_{\text {eqwi }}\right)$ versus optimally allocated time-points $\left(D_{\text {sipi }}\right)$

\begin{tabular}{|c|c|c|c|c|c|c|c|}
\hline \multirow{2}{*}{$\begin{array}{c}\text { Covariance } \\
\text { Structure }\end{array}$} & & \multicolumn{3}{|c|}{$\begin{array}{c}D_{s} \text {-optimality criterion } \\
\text { correlation } \rho_{H K}\end{array}$} & \multicolumn{3}{|c|}{$\begin{array}{c}A \cup C \text {-optimality criterion } \\
\text { correllation } \rho_{I K}\end{array}$} \\
\hline & & 0.3 & 0.5 & 0.7 & 0.3 & 0.5 & 0.7 \\
\hline \multirow[t]{2}{*}{$\mathrm{CS}$} & $\overline{l_{2}}$ & 0.50 & 0.50 & 0.50 & 0.56 & 0.58 & 0.59 \\
\hline & $e f f\left(D_{\text {eqgwi }} \mid D_{\text {opot }}\right)$ & 1.0000 & 1.0000 & 1.0000 & 0.9743 & 0.9625 & 0.9517 \\
\hline \multirow[t]{2}{*}{ ARI } & $t_{2}$ & 0.50 & 0.50 & 0.50 & 0.51 & 0.51 & 0.50 \\
\hline & $e f f\left(D_{\text {eqgut }} \mid D_{\text {opd }}\right)$ & 1.0000 & 1.0000 & 1.0000 & 0.9995 & 0.9999 & 1.0000 \\
\hline \multirow[t]{2}{*}{$\mathrm{AR} 1+\mathrm{ME}^{(1)}$} & $v_{2}$ & 0.50 & 0.50 & 0.50 & 0.55 & 0.57 & 0.59 \\
\hline & $e f f\left(D_{\text {eqquil }} \mid D_{\text {oppis }}\right)$ & 1.0000 & 1.0000 & 1.0000 & 0.9914 & 0.9834 & 0.9651 \\
\hline
\end{tabular}

(i) The measurement error variance $\sigma_{\varepsilon:}{ }^{2}=0.2$ and serial error variance $\sigma_{\varepsilon}{ }^{2}=0.8$.

Table 6.3 shows that the $D_{s}$-optimal allocation of time-points $\left[0, t_{2}, 1\right]$ is equidistant. Although the $A U C$-optimal choice for $t_{2}$ is larger than 0.5 , the design with equidistant timepoints is highly efficient, i.e the relative efficiency of equidistant versus optimally allocated time-points exceeds 0.95 . Thus, for the design with three repeated measures per subject, equidistant time-points are optimal in terms of the $D_{s}$-criterion and highly efficient in terms of the $A U C$-criterion.

\subsection{Example}

An example is given here to illustrate the numerical results of the previous sections. In a 28 -weeks randomized clinical trial, the effect of adenosine triphosphate (ATP) on body composition, energy intake and energy expenditure in 58 patients with advanced non-smallcell lung cancer (NSCLC) was reported (see Agteresch et al., 1999). Fat mass (FM) was one of the primary outcome measures and was assessed at the start of the study and at 4-week intervals for 28 weeks. The mean difference between the ATP $\left(N_{7}=28\right)$ and control $\left(N_{C}=30\right)$ groups showed a quadratic trend over time. In the control group, an approximately linear decrease of mean fat mass (FM) was observed, whereas in the ATP group, the mean FM first slowly decreased and then increased (see Agteresch et al, 1999, Figure 2). Although their 
paper gives no information about the correct covariance structure, let us assume that a firstorder auto-regressive (AR1) covariance structure is a plausible structure for the fat mass, i.e. observations closer together in time will be higher correlated than observations further apart in time and the total body fat mass will be assessed with no or negligible measurement error. Figure 6.4 shows that the efficiency of the design with $K=8$ equally spaced measures may then be increased by decreasing the number of repeated measures $K$, given a fixed study budget.

As an example, we assume again a linear cost function (6.6), where adding a patient costs ten times as much as adding a repeated measure per patient, i.e. the cost ratio $R=C_{1} / C_{2}$ $=10$. For this cost function and a fixed budget, the optimal number of repeated measures $K_{\text {opt }}$ $=4$, irrespective of the correlation $\rho_{\mathbb{K}}$ between first and last measures (see Figure 6.4). If the number of repeated measures $K$ is decreased from 8 to 4 , the number of subjects $N$ $=C /\left\{C_{2}(10+K)\right\}$ increases from $58\left(N_{T}=28, N_{C}=30\right)$ to $74\left(N_{T}=36, N_{C}=38\right)$. The relative efficiency of the used design with eight equally spaced measures (at the start and after $4,8,12,16,20$ and 24 weeks), versus the optimal design with four equally spaced measures (at the start and after 8,16 and 24 weeks), would then be about 0.82 for $0.1 \leq \rho_{1 K} \leq 0.9$. In other words, if the covariance structure is indeed first-order auto-regressive (AR1), the used design with eight equally spaced measures would require a $[(1-0.82) / 0.82] \times 100 \%=22 \%$ higher budget to obtain the same efficiency as the optimal design with four repeated measures.

\subsection{Discussion}

In the present paper, the effect of adding intermediate measures on the efficiency of treatment effect estimators was studied for a second-order polynomial treatment effect, equidistant time-points and different covariance structures, assuming either a fixed number of subjects (Figure 6.2) or a fixed budget for sampling and measuring subjects (Figures 6.3 6.5 ). The $D_{s}$ - and $c$-optimality criteria were considered, where a $D_{s}$-optimal design minimizes the determinant of the covariance matrix of the treatment effect estimators and a $c$-optimal design minimizes the variance of a linear combination of the parameter estimators. In the present paper, a linear combination was chosen so that if the curves of the treatment and control groups do not intersect for $0<t<1$, the $c$-optimality criterion corresponds to the difference between the areas under these curves $(A U C)$. For a linear cost function and a fixed study budget, the $D_{s}$-and $A U C$-optimal designs were calculated. 
We found that the rewults strongly depended on the assumed covariance structure and were very similar for the two optimality criteria that we used. For a fixed number of subjects, the increase in efficiency by adding intermediate measures was large for a compound symmetric (CS) covariance structure and small for a first-order auto-regressive (AR1) structure. For a first-order auto-regressive covariance structure with measurement error $(\mathrm{AR} 1+\mathrm{ME})$, the benefit of adding intermediate measures increased with increasing amount of measurement error and/or increasing auto-correlation, because the ARI+ME structure then approaches the CS structure. Adding a random intercept to the model hardly affected these resullts.

For a linear cost function and a fixed study budget, i.e. adding intermediate measures is the expense of subjects, the results were in line with those for a fixed number of subjects. If the covariance structure resembles compound symmetry (CS) and the cost per subject is eight or more tímes larger than the cost per repeated measure, a large gain in efficiency can be obtained by measuring each subject more than three times. Otherwise, the design with only three equally spaced measures was highly efficient. Additionally, for the design with three repeated measures, equidistant time-points were optimal in terms of the $D_{s}$-optimality criterion and highly efficient in terms of the AUC-optimality criterion.

Although previous or pilot studies may be utilized to estimate the covariance structure of the data, misspecification of the covariance structure may lead to an inefficient design (see Table 6.2). If the covariance structure is unknown, choosing the optimal design based on a compound symmetric (CS) structure may lead to a highly inefficient design. Choosing the optimal design based on a first-order auto-regressive covariance structure with measurement error (ARI+ME), seems to be the best choice in terms of robustness against misspecification.

In the present paper, we assumed that all planned measures were available. In repeated measures studies, missing data due to dropout are common and more subjects and/or more repeated measures per subject are required to obtain efficient treatment effect estimators. Future research is needed to examine the effect of missing data due to dropout on the optimal combination of sample size and number of repeated measures per subject. 


\section{References}

Agteresch, H. J., Rietveld, T., Kerkhof,, L. G. M., Van den Berg, J. W. O, Wilson, J. H. P., \& Dagnelie, P. C. (2002). Beneficial effects of adenosine triphosphate on nutritional status in advanced lung cancer patients: A randomized clinical trial, Jourhal of Clinical Oncology, 20, 371-378.

Atkinson, A. C., \& Donev, A. N. (1996). Optimum Experimental Designs. Oxford: Clarendon Press.

Bischoff, W. (1995). Determinant formulas with applications to designing when the observations are correlated, Ammals of the Institute of Statistical Mathematics, 47, 385399.

Bloch, D. A. (1986). Sample size requirements and the cost of a randomized clinical trial with repeated measurements, Statistics in Medicine, 5, 663- 667 .

Diggle, P. J., Liang, K-Y, \& Zeger, S. L. (1996). Analysis of Longitudinal Data. Oxford: Oxford University Press.

Huang, M-N. L., Chang, F-C, \& Wong, W. K. (1995). D-optimal designs for polynomial regression without an intercept, Sfaristica Simica, $5,441-458$.

Lui, K-J., \& Cumberland, W.G. (1992). Sample size requirement for repeated measurements in continuous data, Statistics in Medicine, 11, 633-641.

Matthews, J. N. S., Altman, D. G., Campbell, M. J., \& Royston, P. (1990). Analysis of serial measurements in medical research, British Medical Journal, 300, 230-235.

Moerbeek, M., van Breukelen, G. J. P., \& Berger, M. P. F. (2000). Design Issues for Experiments in Multilevel Populations, Jowrial of Educational and Behowioral Statistics, 25, 271-284.

Overall, J. E., \& Doyle, S. R. (1994). Estimating sample sizes for repeated measurement designs, Controlled Climical Trials, 15, 100-123.

Pham, B., Cranney, A., Boers, M., Verhoeven, A. C. Wells, G., \& Tugwell, P. (1999). Validity of area-under-the-curve analysis to summarize effect in rheumatoid arthritis clinical trials, Joumal of Rheumatology, 26, 712-716.

Raudenbush, S. W. \& Liu, X-F. (2001). Effects of study duration, frequency of observation, and sample size on power in studies of group differences in polynomial change, Psychological Methods, 6, 387-401.

Schiff, M. (2003). A rationale for the use of summary measurements for the assessment of the effects of rheumatoid arthritis therapies, Cinical Thercapentics, 25, 993-1001. 


\section{Appendix}

\section{$D_{3}$-optimal allocation of time-points $\left[0, t_{2}, 1\right]$ for a CS structure.}

For a CS ( $=\mathrm{RI}+\mathrm{ME})$ structure and 3 repeated measures; model $(6.1)$ can be reduced to

$$
\begin{gathered}
y_{i}=X_{i} \boldsymbol{p}+Z b_{i}+e_{i,} \\
\left(\begin{array}{l}
y_{i 1} \\
y_{i 2} \\
y_{i 3}
\end{array}\right)=\left(\begin{array}{ccccc}
1 & 0 & 0 & 0 & 0 \\
0 & 1 & 0 & G \times t_{2} & G \times t_{2}^{2} \\
0 & 0 & 1 & G & G
\end{array}\right)\left(\begin{array}{l}
\mu_{i 1} \\
\mu_{3} \\
\mu_{3} \\
\beta_{i} \\
\beta_{2}
\end{array}\right)+\left(\begin{array}{l}
1 \\
1 \\
1
\end{array}\right)\left(b_{0 i}\right)+\left(\begin{array}{l}
e_{i 1} \\
e_{i 2} \\
e_{i 3}
\end{array}\right)
\end{gathered}
$$

where $b_{0 i}$ and $e_{i j}$ are independently and normally distributed with means 0 and variance $\sigma_{0}{ }^{2}$ and $\sigma_{e}{ }^{2}$, respectively. The variance of $y_{i j}$ is then $\sigma_{0}{ }^{2}+\sigma_{e}{ }^{2}$ and covariance between $y_{i j}$ and $y_{i j^{\prime}}=$ $\sigma_{0}{ }^{2}$. The generalized least squares (GLS) estimator of $\beta$ and its variance are given by equations (6.3) and (6.4), where $\sum_{i=1}^{N} X_{i}^{\prime} V^{-1} X_{i}=N_{C} X_{C}{ }^{\prime} V^{-1} X_{C}+N_{C} X_{T}^{\prime} V^{-1} X_{T}$ and $X_{C}$ and $X_{T}$ are the design matrices $X_{i}$ for control $(G=0)$ and treatment group $(G=1)$. The $D_{s}$-optimality criterion, i.e. determinant of the covariance matrix of the GLS treatment effect estimators $\hat{\beta}_{1}^{\mathrm{GLSS}}$ and $\hat{\beta}_{2}^{\mathrm{OLS}}$, can then be computed

$$
\operatorname{Det}\left[\operatorname{Var}\left(\hat{\beta}_{12}^{\mathrm{al} \alpha \mathrm{s}}\right)\right]=\left(\frac{N_{C}+N_{T}}{N_{C} N_{T}}\right)^{2} \times \frac{\left(3 \sigma_{0}^{2}+\sigma_{e}^{2}\right) \sigma_{e}^{2}}{\sigma_{0}^{2}+\sigma_{*}^{2}} \times \frac{1}{t_{2}^{2}\left(1-t_{2}\right)^{2}}
$$

where $\hat{\beta}_{12}^{\text {oLs }}=\left(\hat{\beta}_{1}^{\text {OLS }}, \hat{\beta}_{2}^{\text {GL. }}\right)^{n}$. This determinant is minimized for $t_{2}=1 / 2$.

This result is in line with the results from Huang, Chang and Wong (1995) and Bischoff ( $(1995)$ ). For an independence covariance structure, the $D_{s}$-optimal design for model (A1) is supported on two time-points in design space $[0,1]$, namely $t=1 / 2$ and $t=1$ (see Huang, Chang and Wong, 1995, Lemma 1 and Theorem 1). In the present paper, we assumed a third time-point $(t=0)$ to estimate the mean outcome $\mu_{1}$ at the start of the experiment. Further, for model (Al), the $D_{x}$-optimal designs for independence and compound symmetric structures are the same (Bisschoff, 1.995, corollary 3.1). 
Epilogue 


\section{Chapter 7}

\section{Epilogure}

A repeated measures design is often used in a randomized clinical trial to compare the effectiveness of different treatments over time. Since such a study generally demands a high amount of time and money, the characteristics of its design, i.e. sample size, number of repeated measures per subject, time-points, among others, should be chosen carefully at the design stage.

The selection of an efficient design and analysis method depends on the pattern of treatment effect and the covariance structure of the outcome variable. In this thesis, optimal design issues are discussed for linearly divergent (Chapters 4 and 5) or second-order polynomial treatment effects (Chapter 6) and different covariance structures. Although other treatment effect patterns are possible, they are less prominent in the literature.

A linear mixed effects model is very flexible in modelling different sources of variation and therefore, commonly used for the analysis of repeated measures. It is, however, more complicated than traditional analysis methods, like analysis of covariance (ANCOVA). In Chapter 2, these two approaches were compared with respect to the estimation of an overall treatment effect, i.e. difference in post-treatment expectation between treatment groups. Both methods assume that the data are (multivariate) normally distributed. This assumption may, however, be violated for positively skewed data, e.g. cost of medical care and medical recovery times. The data may then be normalized by a log-transformation. Chapter 3 discussed the effect of log-transforming positively skewed data before using a simple method of analysis, like a two-sample $t$-test ${ }_{p}$ analysis of variance (ANOVA) or covariance (ANCOVA).

In the next section, guidelines regarding the design and the analysis method for randomized clinical trials with repeated measures are offered to practitioners based on the results from this thesis. Subsequently, methodological issues are discussed and suggestions for future research are made.

\subsection{Guidelines}

The results from this thesis depended on the anticipated treatment effect pattern, i.e. whether the treatment effect is linearly divergent or second-order polynomial, and the covariance structure of the outcome variable, which are generally unknown at the design stage of a clinical trial. First, guidelines for randomized clinical trials with repeated measures are 
offered to practitioners, assuming a known covariance structure, a known treatment effect pattern (linearly divergent or second-order polynomial) and no missing data. Second; the assumption of a known covariance structure is dropped. Third, guidelines are presented for clinical trials in which the covariance structure and treatment effect pattern are unknown. Finally, the assumption of no missing data, which is often violated for repeated measures data, is taken into consideration.

\section{Linearly divergent treatment effect}

If the structure resembles first-order auto-regression (AR1) (the correlation between repeated measured decreases relatively fast with increasing time-lag), two repeated measures, i.e. one at the start and one at the end of the study, yield highly efficient (small variance) estimators of linearly divergent treatment effects. In this case, analysis of covariance (ANCOVA) instead of the linear mixed effects approach may be used to analyse the data.

If the covariance structure resembles compound symmetry (CS) (the correlation between repeated measures decreases relatively slowly with increasing time-lag should be measured about five times (depending on the ratio of cost per patient and cost per repeated measure). The first measure should then be taken at the start $(t=0)$ and the other four measures should be obtained at the end of the study $(t=1)$, close to each other in time. It should be noted that a compound symmetric covariance structure is infrequently encountered in practice, because it assumes that the correlation between two measures obtained closely in time is the same as the correlation between the first $(t=0)$ and last measure $(t=1)$. In practice, however, successive observations on the same subject tend to be correlated more higlaly than observations taken further apart in time.

If the covariance structure is unknown, an optimal design based on a first-order autoregressive structure with measurement error $(\mathrm{AR} 1+\mathrm{ME})$ is preferable in terms of robustness against misspecification of the covariance structure. In this case, the optimal design has three repeated measures, i.e. one at the start $(t=0)$ and two at the end of the study $(t=1)$

\section{Second-order polywomial treatment effect}

The design with three equally spaced measures often yields highly efficient treatment effect estimators in terms of the $D_{s}$-optimality criterion (simultaneous confidence region of the linear and quadratic treatment effect estimators) and the difference between the area under the curves $(A U C)$ of the treatment and control groups. If the structure is close to compound 
symmetry (CS) and the cost per subject is eight or more times larger than the cost per repeated measure, however, a design with more than three repeated measures is preferred.

If the covariance structure is unknown, an optimal design based on a first-order autoregressive structure with measurement error (ARI+ME) is preferable in terms of robustness against misspecification of the covariance structure. In this case, each subject should be measured three times at equidistant time-points $(t=0, .50,1)$.

If the treatment effect pattern is unknown at the design stage of a study, the design with four equally spaced measures may be used to check whether the treatment effect is linearly divergent, second-order or higher-order polynomial.

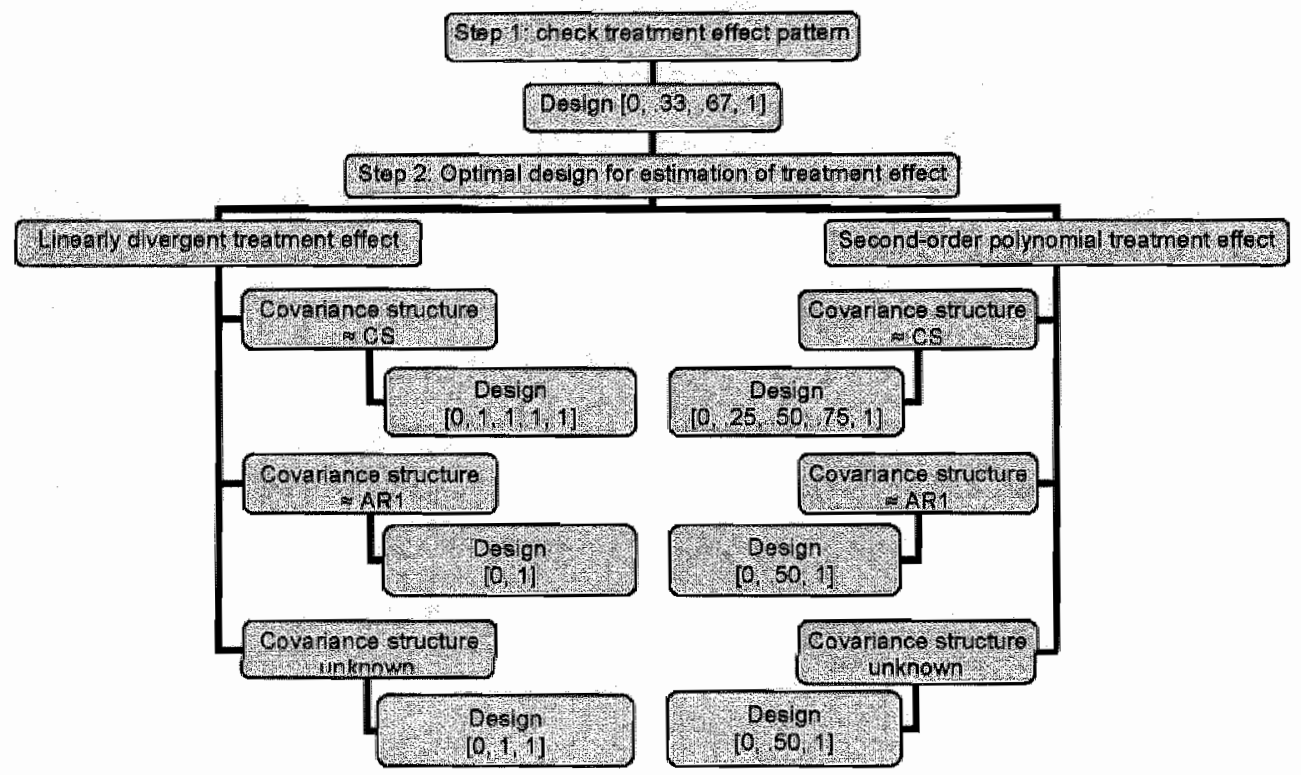

Figure 7.1 Guidelines regarding the design $\left[t_{1}, \ldots, t_{k}\right]$ of randomized clinical trials with repeated measures, assuming no missing data.

The guidelines for the design $\left[t_{1}, \ldots, t_{K}\right]$ of randomized clinical trials with repeated measures are summanized in Figure 7.1. Two steps are distinguished. The first step concerns the check whether the treatment effect pattern is linearly divergent, second-order or higherorder polynomial. In the second step, optimal designs are considered for estimation of the treatment effect parameters. Of course, if the treatment effect pattern is known at the design stage of a study, the first step may be skipped. For example, Figure 7.1 shows that if the treatment effect is linearly divergent and the covariance structure is close to compound 
symmetry $(\approx C S)$, the design $[0,1,1,1,1]$ should be used. This means that each subject should be measured five times, where the first measure is obtained at the start $(t=0)$ and the other measures are taken at the end of the study $(t=1)$, i.e. at time-points that are close to each other.

\section{Missing data}

Although we assumed complete data, missing data due to dropout (subjects who withdraw from the study, so that no further measures for these subjects may be obtained) are common in clinical trials with repeated measures. Three types of missing data mechanisms are defined by Little and Rubin (1987):

1) missing completely at random (MCAR), i.e. the subjects with complete data are indistinguishable from those with incomplete data,

2) missing at random (MAR), i.e. the subjects with complete data differ from those with incomplete data, but the missingness pattern is predictable from variables that are observed, and

3) missing not at random (MNAR), i.e. the pattern of missingness is non-random and is not predictable from variables that are observed, but depends on unobserved variables.

Under MCAR, missing data due to dropout results in power loss, but the treatment effect estimators are still unbiased, whereas under MAR or MNAR, dropout may also result in biased estimators. If dropout is anticipated, this should be taken into account at the design stage, i.e. intermediate measures and/or subjects may be added to the designs recommended above in order to be more robust against missing data and reduce the power loss. If the missing data are MCAR or MAR, a simple method to compute the required sample size to detect a certain treatment effect with sufficient power under dropout is to divide the required sample size under no dropout by the expected proportion of subjects with complete data. For example, if $20 \%$ of the subjects dropout of a study, the required sample size under dropout is $N / 0.8=1.25 \times N$, where $N$ is the required sample size under no dropout. This method is simple, but ignores the dropout process and is very conservative. Methods that incorporate the dropout process (dropout rate at each time-point) into the selection of number of repeated measures per subject and number of subjects may be used to reduce the costs of a study (e.g. Galbraith \& Marschner, 2002; Verbeke \& Lesaffre, 1999). This selection depends on the dropout process and the relative cost per repeated measure and cost per subject. 


\section{Chapter 7}

\subsection{Future research}

Further research is needed to study the effect of dropout and misspecification of the covariance structure and treatment effect pattern on optimal design issues. If the treatment effect pattern is unknown at the design stage of a study, the design with four or five equally. spaced measures may be considered to check whether the treatment effect is linearly divergent, second-order or higher-order polynomial. Misspecification of the treatment effect pattern may lead to inefficient designs (e.g. Berger \& Tan, 2004; Tan \& Berger, 1999).

The guidelines presented in the previous section were based on covariance structures that were assumed to be homogeneous across groups. In practice, heterogeneous covariance structures may be more plausible. In particular, the treatment groups may have the same structure, e.g. AR1, but with different covariance parameter values. This probably affects the optimal ratio of group sizes $N_{t} / N_{c}$ (more subjects to the group with the largest variance and fewer subjects to the group with the smallest variance) rather than the optimal sample size ( $N_{\mathrm{f}}$ $+N_{c}$ ), the optimall number of repeated measures per subject or the optimal allocation of timepoints.

Finally, other (non-stationary) covariance structures than those discussed in this thesis may be considered. For example, an AR1 structure with a heterogeneous variance across time-points (e.g. Verbeke and Molenberghs, 2000, p. 99) is commonly used in practice. The variance formula of the GLS treatment effect estimator may then be complicated and many choices need to be made with respect to the covariance parameters. 


\section{References}

Berger, M. P. F. \& Tan, F. E. S. (2004). Robust designs for linear mixed effects models, Joumal of the Royal Statistical Society, Series C, 53, 569-581.

Galbraith, S. \& Marschner, I. C. (2002). Guidelines for the design of clinical trials with llongitudinal outcomes, Controlled Clinical Trials, 23, 257-273.

Little, R. J. A., \& Rubin, D. B. (1987). Statistical Analysis with Missing Data. New York: John Wiley \& Sons.

Tan, F. E. S., \& Berger, M. P. F. (1999). Optimal allocation of time points for the random effects model, Communications in Statistics, Simulations and Computanions, 28, 517 540.

Verbeke, G., \& Lesaffre, E. (1999). The effect of drop-out on the efficiency of longitudinal experiments, Jownal of the Royal Statistical Society, Series $C_{x}, \mathbf{4 8}, 363-375$.

Verbeke, G., \& Molenberghs, G. (2000). Linear Mixed Models for Longirudinal Data. New York: Springer-Verlag. 
Chapter 7 


\section{Summary}

\section{Summary}

The purpose of the present thesis is to offer guidelines to practitioners for the design and analysis of randomized clinical trials with repeated measures, assuming either a linearly divergent or second-order polynomial treatment effect. The sample size, the number of repeated measures per subject and the allocation of time-points at which the measures are taken can be chosen optimally at the design stage. A design that yields maximally efficient treatment effect estimators is then called an optimal design. Optimal design issues are discussed in Chapters 1,4,5 and 6, while the selection of an analysis method is discussed in Chapters 1, 2 and 3.

Chapter 1 is introductory. It discusses two treatment effect patterns: linearly divergent and second-order polynomial treatment effects. The former is characterized by a linearly increasing difference between the treatment groups ower time. Alternatively, if the difference first increases rellatively fast and then stabilizes or even decreases over time, the treatment effect may be represented by a second-order polynomial function. The chapter proceeds with the description of linear mixed effects model. This model is very usefull for the analysis of repeated measures data, because it is very flexible in modelling different components of variation, like random effects, serial correlation and measurement error. Different covariance structures may be obtained with these components. The generalized least squares (GLS) method is the most commonly used estimation method for the fixed effects in this model. Chapter 1 ends by describing simple analysis methods, like analysis of covariance (ANCOVA), and introducing the log-transformation to normalize positively skewed data. The difference between a linear mixed effects and an ANCOVA model is explained. Both models assume that the data are normally distributed. This assumption is, however, often violated for positively skewed data ${ }_{5}$ e. costs of medical care. A log-transformation may then be used to normalize the data.

If each subject is measured twice, i.e. once before treatment (pre-treatment) and once after treatment (post-treatment), the pre- and post-treatment measures may be treated as a bivariate outcome variable in a linear mixed effects model or the pre-treatment may be treated as a covariate in an analysis of covariance (ANCOVA) model. In Chapter 2, ANCOVA. models are compared with linear mixed effects models with respect to the estimation of a overall treatment effect, i.e. difference in post-treatment expectations between the groups, Four different situations are considered: 
I. the regression slopes and residual post-treatment variances in an ANCOVA model are both homogeneous across groups,

II: heterogeneous regression slopes and homogeneous residual post-treatment variances,

II homogeneous regression slopes and heterogeneous residual post-treatment variances,

IV. heterogeneous regtession slopes and residual post-treatment variances.

If the slopes are homogeneous (situation I and III), ANCOVA is preferred to the linear mixed effects approach, because it yields unbiased treatment effect and variance estimators. If the slopes are heterogeneous and the residual variances are homogeneous (situation II), a linear mixed effects model makes assumptions that are too complicated to apply with the present software, and ANCOVA should be used. Finally, if the slopes and residual variances are both heterogeneous (situation IV), a linear mixed effects model is preferred to an ANCOVA model, since it takes into account the variability of the pre-treatment mean estimator.

Many statistical methods of analysis assume that the outcome variable is normally distributed. This assumption is violated for positively skewed data, which are often encountered in practice. For example, costs for medical care and medical recovery times can only take on positive values and are often affected by positive outliers (high costs or slow recovery). A log-transformation may then be used to normalize the data Chapter 3 discusses the effect of log-transforming positively skewed data on the power of a two-sample t-test, analysis of variance (ANOVA) or covariance (ANCOVA). It is shown that the required sample size to detect a treatment effect with a certain power may be decreased by $\log$ transforming the data. This decrease in sample size can be substantial if the coefficient of variation (CV), i.e. the standard deviation divided by the mean, of the untransformed data is large.

Chapter 4 discusses the effect of adding intermediate measures on the variance of the generalized least squares (GLS) treatment effect estimator for six different covariance structures, assuming a linearly divergent treatment effect, equidistant time-points and either a fixed sample size or a fixed study budget. It shows that this effect strongly depends on the covariance structure of the repeated measures. The decrease in variance by adding intermediate, equally spaced measures is relatively large for a fixed sample size if

a) the covariance structure is compound symmetric or

b) the structure is close to compound symmetry and the correlation between two repeated measures does not exceed 0.80 or

c) the correlation does not exceed 0.60 , if the time-lag between the measures goes to zero. 


\section{Swmory}

For a fixed budget and a linear cost function, the design with two repeated measures per subject, i.e. one at the start and one at the end of the study, often yield highly efficient (small variance) treatment effect estimators. Only in the special case where the covariance structure resembles compound symmetry and the cost per subject is ten or more times larger than the cost per repeated measure, are more than two repeated measures required to obtain this goal. The covariance structure is, however, often unknown at the design stage of a study. In that case, an optimal design based on a first-order auto-regressive stnucture with measurement error $(A R 1+M E)$ should be used to be robust against misspecification of the covariance structure.

Repeated measures are often planned to be equidistant, but this does not always yield highly efficient treatment effect estimators. In Chapter 5, the optimal allocation of time-points is calculated and compared with equidistant time-points for a small number of repeated measures, different covariance structures and linearly divergent treatment effects. The effect of adding optimally spaced measures (at the expense of subjects) is also investigated. The assumed covariance structure is crucial for the results. Firstly, for covariance structures resembling compound symmetry (the correlation between repeated measures decreases relatively slowly with increasing time-lag), each subject should be measured about five times and equidistant time-points do not yield highly efficient treatment estimators. The first measure should then be taken at the start and the other measures should be obtained at the end of the study, close to each other in time. Secondly, if the covariance structure resembles firstorder auto-regression (the correlation between repeated measures decreases relatively fast with increasing time-lag), two repeated measures, i.e. one at the start and one at the end of the study, are sufficient to obtain highly efficient treatment effect estimators. Finally, if the covariance structure is unknown at the design stage of a study, an optimal design based on a first-order auto-regressive structure with measurement error is preferable in terms of robustness against misspecification of the covariance structure. In this case each subject should be measured three times, i.e. once at the start and wice at the end of the study.

Chapter 6 deals with the optimal selection of sample size and the number of repeated measures per subject for clinical trials with equidistant time-points and second-order polynomial treatment effects. Two optimality criteria are considered:

1) a $D_{s}$-optimality criterion, i.e minimize the area of the simultaneous confidence region of the linear and quadratic treatment effect estimators, and

2) a c-optimality criterion, i.e. minimize the variance of a linear combination of the linear and quadratic treatment effect estimator. A linear combination is chosen so that it 
corresponds to the difference between the areas under the curves of the treatment and control groups (AUC).

The effect of adding intermediate measures (at the expense of subjects) on the efficiency of the treatment effect estimators depends strongly on the assumed covariance structure and is hardly affected by the choice between the two optimality criteria. Given a fixed number of subjects, the increase in efficiency by adding intermediate, equally spaced measures is large for covarance structures close to compound symmetry and small for structures that resemble first-order auto-regression. For a fixed study budget and a linear cost function, the design with only three equally spaced measures is often highly efficient. Only if the structure resembles compound symmetry and the cost per subject is eight or more times larger than the cost per repeated measure, each subject should be measured more than three times. On the other hand, if the covariance structure is unknown, the design with three equally spaced measures should be used. Given a design with three repeated measures and a second-order polynomiall treatment effect, equidistant time-points are $D_{s}$-optimal and highly efficient in terms of the $A U$ C-optimality criterion.

Finally, Chapter 7 (epilogue) summarizes the results of this thesis and offers guidelines regarding the design and method of analysis for randomized clinical trials with linearly divergent or second-order polynomial treatment effects. 


\section{Samenvatting in het Nederlands (Dutch summary)}

Als resultaat van dit proefschrift worden in hoofdstuk 7 (epiloog) een aantal praktische richtlijnen aangeboden m.b.t. het opzetten en analyseren van gerandomiseerde klinische onderzoeken met herhaalde metingen. We nemen hierbij aan dat het effect van een. behandeling of medicijn linear toeneemt in de tijd of te beschrijven is met een kwadratische functie. Het aantal individuen, het aantal metingen per individu en de tijdstippen waarop de metingen genomen worden kunnen optimaal gekozen worden tijdens de opzetfase van een onderzoek. Een onderzoeksopzet is optimaal wanneer deze leidt tot maximaal efficiente behandelingsschatters. Hoofdstukken 1, 4,5 en 6 gaan over het optimaal opzetten van gerandomiseerde klinische onderzoeken, terwijll de selectie van een analyse-methode wordt besproken in de hoofdstukken 1, 2 en 3.

Hoofdstuk 1 is inleidend van aard. Hierin worden twee behandelingseffecten. behandeld: lineair divergerende en kwadratische. Het eerste behandelingseffect wordt gekenmerkt door een verschil tussen twee behandelingsgroepen dat lineair toeneemt in de tijd. Anderzijds, als het verschil eerst relatief snel toeneemt en daarna stabiliseert of zelfs afneemt, dan spreken we van een kwadratisch behandelingseffect. In het hoofdstuk wordt vervolgens een lineair mixed effects model beschreven. Het analyseren van herhaalde metingen data is erg gemakkelijk met dit model, omdat het erg flexibel is in het modelleren van verschillende variatiecomponenten, zoals random effecten, serieële correlatie en meetfouten. Verschillende covariantie structuren kunnen verkregen worden met deze variatiecomponenten en de generaliseerde kleinste kwadraten (Engels: generalized least squares; GLS) schattingsmethode is de meest gebruikte methode om de fixed effecten mee te schatten in een lineair mixed effects model. Hoofdstuk 1 eindigt met het beschrijven van eenvoudige analyse methoden, zoals covariantie-analyse (Engels: analysis of covariance; ANCOVA), en met het introduceren van de log-transformatie om positief scheve data te mormaliseren. Het versohil tussen een lineair mixed effects en een ANCOVA model wordt verklaard. Beide modellen nemen aan dat de data normaal verdeeld zijn. Deze aanname is echter vaak geschonden voor positief scheve data, b.v. de kosten woor medische zorg. Een log-transformatie kan dan gebruikt worden om de data te normaliseren.

Als elk individu twee keer is gemeten, $d . w, z$. een keer voor behandeling (voormeting) en een keer na behandeling (nameting), dan kan de voor- en nameting als een bivariate uitkomstvariabele opgenomen worden in een lineair mixed effects model of de voormeting kan als covariaat meegenomen worden in een covariantie-analyse (ANCOVA) model. In 


\section{Dutch summary}

hoofdstuk 2 worden ANCOVA modellen vergeleken met lineair mixed effects modellen ta.v. het schatten van een algeheel behandelingseffect, d.w.z. het verwachte werschill tussen de groepen op de nameting. Vier verschillende situaties worden behandeld:

1. de regressiehellingen en residuele varianties in een ANCOVA model zijn beiden homogeen over de groepen,

II. heterogene regressiehellingen en homogene residuele varianties,

III. homogene regressiehellingen en heterogene residuele varianties,

IV. heterogene regressiehellingen en residuele varianties.

Als de hellingen homogeen zijn (situaties I en III), dan verdient ANCOVA de voorkeur boven de lineair mixed effects methode, omdat ANCOVA leidt tot zuivere behandelingseffect- en variantieschatters. Als de hellingen heterogeen zijn en de residuele varianties homogeen (situatie I), dan maakt een lineair mixed effects model aannames die te ingewikkeld zijn om gemodelleerd te worden met de huidige software. ANCOVA zal dan gebruikt moeten worden. Tenslotte als de hellingen en residuele varianties beiden heterogeen zijn over de groepen (situatie IV), dan verdient een lineair mixed effects model de voorkeur boven een ANCOVA model, want alleen de eerste houdt rekening met de variabiliteit van de schatter van het populatiegemiddelde op de voormeting.

Veel statistische analysemethoden nemen aan dat de uitkomstvariabele normaal. verdeeld is. Deze aanname is geschonden voor positief scheve data. Kosten voor medische zorg en medisch hersteltijden zijn voorbeelden van positief scheve data, omdat deze alleen positieve waarden kunnen aannemen en vaak uitschieters hebben naar boven (hoge kosten of langzaam herstel). Een log-transformatie kan dan gebruikt worden om deze data te normaliseren. Hoofdstuk 3 bespreekt het effect van het log-transformeren van positief scheve datta op het vermogen van een twee-steekproeven $t$-toets, variantie-analyse (Engels: analysis of variance; ANOVA) of covariantie-analyse (ANCOVA). In dit hoofdstuk is aangetoond dat het vereiste aantal proefpersonen om een behandelingseffect te detecteren met een bepaald vermogen kan dalen door een log-transformatie. Deze daling in steekproefgrootte kan erg substantièel zijn als de variatie-coefficiènt (Engels: coefficient of variation; CV) van de ongetransformeerde data groot is, d.w.z. de standaard deviatie is groot t.o.v. het gemiddelde.

Hoofdstuk 4 bespreekt het effect van het toevoegen van tussenliggende metingen op de variantie van de generenaliseerde kleinste kwadraten (GLS) schatter van het behandelingseffect, waarbij zes covariantie structuren worden bekeken. Er wordt aangenomen dat het behandelingseffect lineair divergerend is, de tijdstippen waarop gemeten wordt op gelijke afstand van elkaar liggen en dat de steekproefgrootte of het studie-budget vastligt. We 


\section{Dwh swomary}

hebben aangetoond dat dit effect erg afhangt van de covariantie-structuur van de herhaalde metingen. De daling in variantie door het toevoegen wan tussenliggende, op gelijke afstand genomen metingen is relatief groot bij een vooropgestelde steekproefgrootte, als

a) de covariantie structur compound symmetrisch is of

b) de structuur dichtbij een compound symmetrische structuur ligt en de correlatie tussen twee herhaalde metingen altijd kleimer is dan 0.80 of

c) de correlatie nooit groter is dan 0.60 , zelfs niet als de tijdsspanne tussen twee metingen naar 0 gaat.

Als het budget vastligt en de kostenfunctie lineair is, dan leidt het onderzoeksopzet waarbij elk individu twee keer wordt gemeten, d.w.z. een keer aan het begin en een keer aan het eind van het onderzoek, tot erg efficiënte schatters van het behandelingseffect. Alleen in het specialle geval waarin de covariantie-structuur lijkt op compound symmetrie en de kosten per individu tien of meer keer hoger is dan de kosten per meting, zijn meer dan twee metingen nodig om dit doel te bereiken. Echter de covariantie-structuur is vaak onbekend in de opzetfase van een onderzoek In dat geval moet een optimaal onderzoeksopzet, dat gebaseerd is op een eerste-orde anto-regressieve covariantie-structuur met meetfout, gebruikt worden om bestand/robuust te zijn tegen het verkeerd specificeren van de covariantie-structuur.

Herhaalde metingen worden vaak gepland met gelijke afstand tussen de metingen, maar dit leidt niet altijd tot heel efficiente schatters van het behandelingseffect. In hoofdstuk 5 is de optimale ligging van tijdstippen uitgerekend en vergeleken met gelijk-verdeelde tijdstippen (gelijke afstand tussen de metingen), waarbij verschillende covariantie-structuren worden bekeken. Verder is aangenomen dat het aantal metingen per persoon beperkt is en het behandelingseffect lineair divergerend is. Het effect van het toevoegen van optimaal gelegen tussenmetingen (ten koste van het aantal proefpersonen) is ook onderzocht. De aangenomen covariantie-structuur bleek cruciall te zijn voor de resultaten. Ten eerste, als de covariantiestructuur dichtbij compound symmetrie ligt, d.w.z. de correlatie tussen herhaalde metingen daalt relatief langzaam met het toenemen van de tijdsspanne tussen de metingen, dan moet elk individu ongeveer vijf keer gemeten worden, waarbij de eerste meting aan het begin genomen moet worden en de andere vier, kort na elkaar, aan het eind van het onderzoek. Ten tweede, als de covariantie-structuur dichtbij eerste-orde auto-regressie ligt, d.w.z. de correlatie tussen herhaalde metingen daalt relatief snel met het toenemen van de tijdsspanne tussen de metingen, dan zijn twee metingen per individu, d.w.z. een meting aan het begin en een aan het. eind van het onderzoek, voldoende om heel efficiënte schatters van het behandelingseffect te krijgen. Tenslotte, als de covariantie-structuur onbekend is in de opzetfase van een onderzoek, 
dan verdient een optimale onderzoeksopzet gebaseerd op een eerste-order auto-regressieve cowariantie-structuur met meetfout de voorkeur t.a. $\mathrm{v}$. de robuustheid tegen het verkeerd specificeren van de covariantie-structuur. In dit geval moet elk individu drie keer gemeten worden, een keer aan het begin en twee keer, kort na elkaar, aan het eind van het onderzoek.

Hoofdstuk 6 behandelt de optimale selectie van de steekproefgrootte en thet aantal metingen per individu voor klinische experimenten, waarin de herhaalde metingen met gelijke afstand ertussen genomen zijn en het behandelingseffect een kwadratische functie van de tijd is. Twee optimaliteitscriteria zijn gebruikt:

1) een $D_{s}$-optimaliteitscriterium, d.w.z. minimaliseer de oppervlakte van het simultane betrouwbaarheidsgebied van de lineaire en kwadratische behandelingseffectschatters,

2) een c-optimaliteitscriterium, d.w.z. minimaliseer de variantie van een lineaire combinatie van de lineaire en kwadratische behandelingseffectschatters. Een lineaire combinatie is gekozen zodat deze overeenstemt met het verschil tussen de oppervlakten onder de curves (Engels: areas under the curves; AUC) van de behandelings- en controlegroep.

Het effect van het toevoegen van tussengelegen metingen (ten koste van het aantal proefpersonen) op de efficiëntie van de schatters van het behandelingseffect hangt sterk af van de aangenomen covariantie-structurur en wordt nauwelijks beïnvloed door de keuze tussen de twee optimaliteitscriteria. Gegeven een vast aantal proefpersonen, de toename in efficièntie door het toevoegen van tussengellegen, op gelijke afstand van elkaar genomen metingen is groot wanneer de covariantie-structuur dichtbij compound symmetrie ligt en deze is klein wanneer de structuur dichtbij eerste-order auto-regressie ligt. Als het studiebudget vastligt en de kostenfunctie lineair is, dan is de onderzoeksopzet, waarbij elk individu slechts drie keer genteten is, vak erg efficiënt. Alleen wanneer de structuur dichtbij compound symmetrie ligt en de kosten per individu acht of meer keer hoger is dan de kosten per meting, dan moet elk individu vaker dan drie keer gemeten worden. Anderzijds, als de covariantie-structuur onbekend is, dan moet de onderzoeksopzet met drie herhaalde metingen en gelijke afstand tussen de metingen gebruikt worden. Gegeven een onderzoeksopzet met drie herhaalde metingen en een kwadratisch behandelingseffect, gelijk-verdeelde tijdstippen zijn $D_{\mathrm{g}}$-optimaal en heel efficiènt in termen van de $A U C$-optimaliteitscriterium.

Tenslotte, hoofdstuk 7 (epiloog) vat de resultaten van dit proefschrift samen en biedt guidelines aan die betrekking hebben op het opzetten en analyseren van gerandomiseerde klinische experimenten warin het behandelingseffect lineair divergerend of een kwadratische functie van de tijd is. 


\section{Curricuhm Vilae}

\section{Curriculum Vìtae}

Bjorn Winkens was born on March 4, 1977 in Oirsbeek, the Netherlands. From 1989 to 1995 he attended his secondary education at the Rombouts College in Brunssum. Subsequently he started studying mechanical engineering at the Zuyd University in Heerlen. After one year he switched to mathematics at the University of Nijmegen, graduating in 2001, with specialisation in applied statistics. In the same year, after successfully attaining his master"s degree, he was accepted as a Ph.D. student at the department of Methodology and Statistics, University of Maastricht. The year 2004 made him the happiest man on earth, when be married his beautiful girlfriend, Aline, and became father of the most beautiful baby girl, Isis. In July 2005 he started working as a study coordinator at the department of Medical Statistics, at Aachen University (RWTH), Germany. 